PNL - 4679

NUREG/CR-3196

PNL-4679

BHARC-400/83/003

\title{
Drug and Alcohol Abuse:
}

The Bases for Employee Assistance Programs in the Nuclear Utility Industry

Prepared by L. R. Radford, W. L. Rankin, V. Barnes, M. V. McGuire, A. M. Hope

Battelle - Human Affairs Research Centers

Pacific Northwest Laboratory

Operated by

Battelle Memorial Institute

Prepared for

U.S. Nuclear Regulatory

Commission 


\section{NOTICE}

This report was prepared as an account of work sponsored by an agency of the United States Government. Neither the United States Government nor any agency thereof, or any of their employees, makes any warranty, expressed or implied, or assumes any legal liability of responsibility for any third party's use, or the results of such use, of any information, apparatus, product or process disclosed in this report, or represents that its use by such third party would not infringe privately owned rights.

\section{Availability of Reference Materiais Cited in NRC Publications}

Most documents cited in NAC publications will be available from one of the following sources:

1. The NRC Public Document Room, 1717 H Street, N.W. Washington, DC 20555

2. The NAC/GPO Sales Program, U.S. Nuclear Regulatory Commission, Washington, DC 20555

3. The National Technical Information Service, Springfield, VA 22161

Although the listing that follows represents the majority of documents cited in NRC publications, it is not intended to be exhaustive.

Referenced documents available for inspection and copying for a fee from the NRC Public Document Room include NRC correspondence and iriternal NAC memoranda; NRC Office of Inspection and Enforcement bulletins, circulars, information notices, inspection and investigation notices; Licensee Event Reports; vendor reports and correspondence; Commission papers; and applicant and licensee documents and correspondence.

The following documents in the NUREG series are available for purchase from the NRC/GPO Sales Program: formal NRC staff and contractor reports, NRC-sponsored conference proceedings, and NRC booklets and brochures. Also available are Regulatory Guides, NRC regulations in the Code of Federal Regulations, and Nuclear Regulatory Commission Issuances.

Documents available from the National Technical Information Service include NUREG series reports and technical reports prepared by other federal agencies and reports prepared by the Atomic Energy Commission, forerunner agency to the Nuclear Regulatory Commission.

Documents available from public and special technical libraries inciude all open literature items, such as books, journal and periodical articles, and transactions. Federal Register notices, federal and state legisiation, and congressional reports can usually be obtained from these libraries.

Documents such as theses, dissertations, foreign reports and translations, and non-NRC conference proceedings are available for purchase from the organization sponsoring the publication cited.

Single copies of NAC draft reports are available free upon written request to the Division of Technical Information and Document Control, U.S. Nuclear Regulatory Commission, Washington, DC 20555

Copies of industry codes and standards used in a substantive manner in the NRC regulatory process are maintained at the NRC Library, 7920 Norfolk Avenue, Bethesda, Maryland, and are available there for reference use by the public. Codes and standards are usually copyrighted and may be purchased from the originating organization or, if they are American National Standards, from the American National Standards Institute, 1430 Broadway, New York, NY 10018. 
NUREG/CR-3196

PNL-4679

BHARC-400/83/003

$\mathrm{RX}$

\section{Drug and Alcohol Abuse: The Bases for Employee Assistance Programs in the Nuclear Utility Industry}

Manuscript Completed: June 1983

Date Published: July 1983

Prepared by

L. R. Radford, W. L. Rankin, V. Barnes, M. V. McGuire, A. M. Hope

Battelle - Human Aftairs Research Centers

Seattle, WA 98105

Under Contract to:

Pacific Northwest Laboratory

Richland, WA 99352

\section{Prepared for}

Division of Facility Operations

Office of Nuclear Regulatory Research

U.S. Nuclear Regulatory Commission

Washington, D.C. 20555

NRC FIN B2453 



\section{ABSTRACT}

This report describes the nature, prevalence, and trends of drug and alcohol abuse among members of the U.S. adult population and among personnel in non-nuclear industries. Analogous data specific to the nuclear utility industry are not available, so these data were gathered in order to provide a basis for regulatory planning. The nature, prevalence, and trend information was gathered using a computerized 1 iterature search, telephone discussions with experts, and interviews with employee assistance program representatives from the Seattle area. This report also evaluates the possible impacts that drugs and alcohol might have on nuclear-related job performance, based on currently available nuclear utility job descriptions and on the scientific literature regarding the impairing effects of drugs and alcohol on human performance. Employee assistance programs, which can be used to minimize or eliminate job performance decrements resulting from drug or alcohol abuse, are also discussed. 

ABSTRACT

CONTENTS

LIST OF TABLES

FOREWORD

ACKNOWLEDGEMENTS.

EXECUTIVE SUMMARY

1. INTRODUCTION • • • • • • • • • • • • • • • • • • • 1

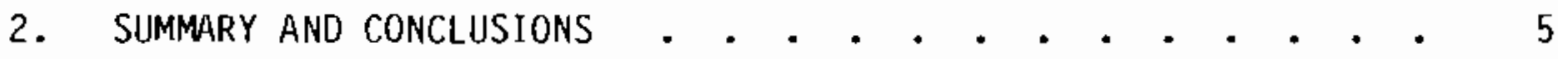

2.1 Drug Abuse . • . . . . . . . . . . . . . 6

2.2 Alcohol Abuse . . . . . . . . . . . . . . . 7

2.3 Mult iple Substance Abuse . . . . . . . . . . . 7

2.4 Nuclear Utility Industry . . . . . . . . . . . 7

2.5 Evaluation of Impairing Effects . . . . . . . . . 8

3. DEFINITION OF DRUG ABUSE AND ALCOHOL ABUSE . • • • • . • • 10

4. NATURE, PREVALENCE, ANO TRENDS OF DRUG ABUSE

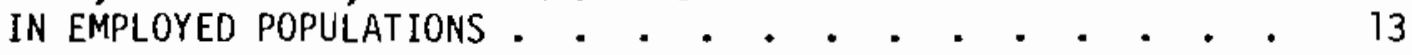

4.1 Factors Influencing Drug Abuse Patterns . . . . . . 13

4.2 General Population Survey Findings on Drug Abuse . . . 15

4.3 Nuclear Licensee Survey Findings for Drug Abuse . . . . 18

4.4 Drug Abuse in Non-nuclear Industries . . . . . . . . 19

4.5 Summary and Conclusions . . . . . . • . . . . . 22

5. IMPAIRING EFFECTS OF DRUG ABUSE ON JOB PERFORMANCE $\quad \cdot \quad \cdot \quad \cdot \quad 23$

5.1 Model of Human Performance - . . . . . . . . . . 23

5.2 Nuclear Ut ility Job Categories as Related
to the Model of Human Performance . . . . . . . 24

5.2 .1 Managers . . . . . . . . . . . . . . 25

5.2.2 Scientists and Engineers . . . . . . . . . 25

5.2 .3 Licensed Operators . . . . . . . . . . . 25

5.2.4 Non-licensed Operators . . . . . . . . . 28

5.2.5 Skilled Craft Workers . . . . . . . . . 28

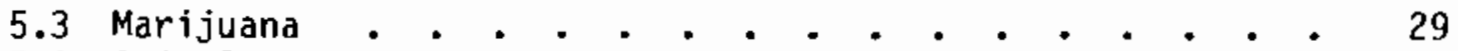

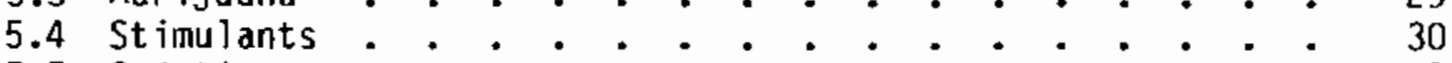

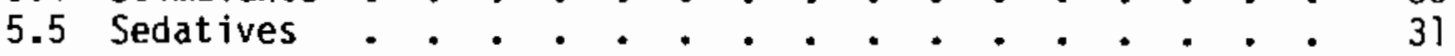

5.5.1 Anxiolytics . . . . . . . . . . . . 32

5.5 .2 Barbiturates . . . . . . . . . . . . . 32

5.5.3 Interactive Effects of Sedatives . . . . . . 33

5.5 .4 Summary. . . . . . . . . . . . . . . 34 
5.6 Hallucinogens . . . . . . . . . . . . . . 34

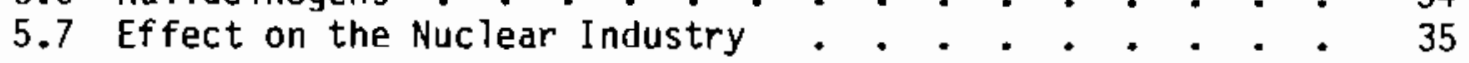

6. NATURE, PREVALENCE, AND TRENDS OF ALCOHOL ABUSE

IN EMPLOYED POPULATIONS . . . . . . . . . . . . . . . . 39

6.1 Factors Influencing Alcohol Abuse Patterns . . . . . 39

6.2 Alcohol Abuse Prevalence Rates in the Nuclear Industry Predicted Using the Marden Formula . . . . 40

6.3 Nuclear Licensee Survey Findings on Alcohol Abuse $: \dot{*} 44$

6.4 Alcohol Abuse in Non-nuclear Industries . . . . . . 44

6.5 Summary and Conclusions. . . . . . . . . . . 48

7. IMPAIRING EFFECTS OF ALCOHOL ABUSE ON JOB PERFORMANCE • • • 50

7.1 Impairing Effects of Alcohol . . . . . . . . . 50

7.2 Effect on the Nuclear Industry . . . . . . . . 53

8. CONCLUSIONS ANO RECOMMENDATIONS . . . . . . . . . . . 55

9. REFERENCES . • • • • • • • • • • • • • . . 57

APPENDIX A: Interview Format for Utility Survey . . . . . . . 62

APPENDIX B: Local Industries Surveyed . • • • • • • • • • 71

APPENDIX C: Impairing Effects of Drugs on Human Performance . • 72

APPENDIX D. Impairing Effects of Alcohol on Human Performance . $\quad 89$

APPENDIX E. Bibliography . • . • • . • • . • . • • • 97 


\section{LIST OF TABLES}

Table

Page

1 Current Marijuana Abuse Among Young (Age 18-25)

and 01der (Age 26+) Adults by Region and

Population Density: 1976-1979......... . . 14

2. Drug and Alcohol Lifetime Prevalence Rates and

Past Month Prevalence Rates for the U.S. Population

3. Most Frequently Abused Drugs by Employees on

the Job, Ranked in Order of the Most Common

Problem (1) to the Least (3) . . . . . . . . . . 21

4. Human Performance Requirements of Major

Utility Jobs . • . . . . . . • . • . • . • • 26

5. Estimated Impact of the Impairing Effects of Four

Types of Orugs on Job Performance for Five

Occupational Categories . . . . . . . . . . . . 38

6. Marden Formula Probabilities for Alcohol Abuse

Based on Sex and Age . . . . . . . . . . . . . 42

7. Marden Formula Probabilities for Alcohol Abuse

Among Men Based on Age and 0ccupation . . . . . . . 43

8. The Relationship of Nuclear Power Plant Job

Categories to the Occupational Categories Used

in the Marden Formula . . . . . . . . . . . . 45

9. Employees Reporting Drug-Alcohol Problems

Over Past Five Years in Selected Sample of

Industries

10. Relationship Levels of Blood Alcohol Concentrations

and Types of Performance Decrements . . . . . . .

11. Predicted Negative Impact of Alcohol on the High

Frequency Performance Areas for Five

Occupational Categories 



\section{FOREWORD}

Drug and alcohol abuse among the general workforce of the United States has been recognized as a significant concern since the $1950^{\circ} \mathrm{s}$. Its major impact on industry has been in the areas of industrial safety and productivity. Two rules to the Code of Federal Regulation have been proposed--the "Access Authorization Rule" (10 CFR 73.56) and the "Fitness for Duty Rule" (I0 CFR 50.54). In order to provide a technical basis for possible regulatory action on these rules, the possible impact of drug and alcohol abuse on the job performance of nuclear utility personnel needs to be estimated. This report is a synthes is of present knowledge about the nature, prevalence, and trends of drug and alcohol abuse among the U.S. population and workforce and about the impairing effects of drugs and alcohol on human performance. Thus, information presented in this report provides part of the technical basis for regulatory planning. In addition, this report provides information about employee assistance programs that can be used in the nuclear utility industry to overcome job performance decrements caused by drug and alcohol abuse. 

Many individuals at Battelle and the Nuclear Requlatory Commission (NRC) read and commented on this report. However, special thanks go to our Battelle manager, Robert $\mathrm{J}$. Sorenson, for the support and guidance that he provided on the project and to our NRC Project Manager, Dr. Thomas $G$. Ryan, for the comments and guidance that he provided. We also wish to thank Charleen Duitsman for this draft and the numerous earlier drafts of this report that she so professionally produced. 



\section{EXECUTIVE SUMMARY}

The original purposes of this report were twofold: (1) to characterize and describe the nature, prevalence, and trends of drug and alcohol abuse in the nuclear utility industry, and (2) to evaluate possible impacts of drug and alcohol abuse on decrements in job performance of nuclear power plant personnel. However, only minimal data specific to the nuclear utility industry are currently available regarding the first purpose. Also, because of resource and time limitations and the sensitivity of drug and alcohol abuse issues, a direct survey of drug and alcohol abuse among nuclear utility industry personnel was not feasible. Thus, this report describes nature, prevalence, and trend issues as determined from non-nuclear industries and from the general, U.S. adult population. Without substantially more investigation, there is no way to determine whether employment practices with in the nuclear utility industry would increase or decrease the prevalence of drug and alcohol abuse compared to the general population or non-nuclear industries. In order to provide a basis for regulatory planning, however, it is reasonable to assume that generally similar results would be found. Possible impacts of drug and alcohol abuse on nuclear-related job performance are identified based on currently available nuclear utility job descriptions and on the scientific literature regarding the impairing effects of drugs and alcohol on human performance.

This information is needed by the Nuclear Regulatory Commission in order to provide guidance in establishing employee assistance programs that are practical, acceptable to government and industry, and demonstrate potential for minimizing/eliminating the use of drugs and alcohol on the job or off the job, if such use significantly degrades job performance.

It has been estimated that $10 \%$ to $12 \%$ of the general workforce experiences job performance decrements as a result of alcohol abuse, mental health and emotional problems, marital/family problems, drug abuse, and other behavioral problems. Employee assistance programs, which have been used to mitigate performance problems due to all causes, including drug and alcohol abuse, have been shown to be $70 \%$ to $90 \%$ effective in treating troubled employees in numerous workplace settings.

Orug Abuse

For purposes of this study, drug abuse is defined as the use of illegal drugs or the illegal use of prescription drugs. Abuse of over-the-counter drugs is not assessed because of lack of data. The drugs of abuse included marijuana, cocaine and other stimulants, sedatives (sleeping pills), analgesics (pain killers), minor tranquilizers, and hallucinogens.

National survey data on a representative sample of the U.S. adult (over 18) population in 1979 show that the drugs most frequently abused 
are marijuana, cocaine and other stimulants, hallucinogens, inhalants, sedatives, minor tranquilizers, and analgesics (in that order). Over half of the non-alcohol drug use involves marijuana. Estimates of the total adult population who are current abusers of at least one drug are between $12 \%$ and $20 \%$. Of those adults reporting drug abuse, it can be estimated that $3 \%$ to $5 \%$ have abused drugs on the job at least once. (This does not mean that they routinely abuse drugs on the job.)

Employee assistance program consultants estimate that approximately $1 \%$ of a given workforce has significant on-the-job drug abuse problems that require the services of an assistance program. Seattle-area employee assistance program representatives estimated that from $4 \%$ to $10 \%$ of those employees who have sought help from the ir program have significant on-the-job drug abuse problems. These figures translate to $0.3 \%$ or less of their total workforce.

A small sample of representatives from nuclear utility employee assistance programs said they were aware of little to no drug abuse at their plants. However, several dozen drug-related arrests at nuclear power plants over the past five years indicate that some drug abuse exists at the plants. The general workforce estimate of drug abuse used by employee assistance progran consultants probably provides the best estimates for regulatory planning purposes.

\section{Alcohol Abuse}

For purposes of this study, alcohol abuse is equated with problem drinking, where a problem drinker is defined as someone who experiences social, psychological, and/or physiological problems as a result of their alcohol intake. Therefore, alcohol abusers include the alcoholic (i.e., one who has the disease of alcoholism) and those who are not alcoholic but who, because of their drinking, experience serious problems to the extent that some sort of intervention is advisable. Alcohol abusers exhibit characteristic behavior patterns and can be reliably identified by them.

National survey data on adult drinking habits collected in the late 1960 's provide the best alcohol abuse prevalence estimates available on the U.S. adult population. These data indicated that approximately $11 \%$ of the U.S. adult population between 20 and 70 years of age are alcohol abusers. Sex, age, and occupation were found to be highly related to alcohol abuse prevalence. Approximately $17 \%$ of the adult male population were found to be alcohol abusers while only $4 \%$ of the adult women were found to be alcohol abusers. In terms of age differences, $13 \%$ of adults from 20 to 29 years, $12 \%$ of adults from 30 to $39,12 \%$ of adults from 40 to $49,7 \%$ of adults from 50 to 59 , and $6 \%$ of aduits from 60 to 69 were a]cohol abusers.

Three job categories that are important in the nuclear utility industry are professional and technical workers, managers and administrators, and craftsmen. General population alcohol abuse prevalence estimates for these job categories for men (no estimates are available for women in the 
given occupations) aged 20 to 69 are $10 \%$ for professional and technical workers, $18 \%$ for managers and administrators, and $21 \%$ for craftsmen.

Employee assistance program consultants estimate that approximately $5 \%$ to $6 \%$ of the total workforce has serious enough alcohol abuse problems to degrade job performance and, therefore, to warrant treatment. For an all-male workforce, this estimate would be revised upward to $8 \%$ to $10 \%$. Seattle-area employee assistance program representatives, surveyed for the purposes of this study, estimated that a minimim of $10 \%$ of their employees are in need of treatment for their alcohol abuse problems. From $45 \%$ to $100 \%$ of those seeking help from Seattle-area employee assistance programs did so specifically for alcohol abuse problems. This translates to less than $1 \%$ of the workforce actually seeking help through the employee assistance program for their alcohol abuse problem.

However, help can also be sought through ways other than the employee assistance program.

We attempted to collect preliminary data on alcohol abuse prevalence specific to the nuclear industry using a telephone interview technique. However, data were only collected from six employee assistance program representatives. Five estimated that $3 \%$ or less of their plant staff were alcohol abusers; the sixth representative estimated that as many as $9 \%$ of the plant staff may suffer from alcohol abuse. Because few records are kept on these utility programs, these estimates were based only upon personal observation or personal opinion.

In our professional opinion, the best estimates of alcohol abuse at nuclear utilities are provided by the occupational category estimates and the general workforce estimates. These data provide a range of estimates of alcohol abuse prevalence that are suitable for making regulatory decisions. Analysis of a range of estimates helps to make up for the fact that the estimates were developed from the general population and that the effects of nuclear industry employment practices on these estimates are unknown.

\section{Mult iple Substance Abuse}

A present and future problem in the U.S. industrial workforce is likely to be multiple substance abuse; that is, alcohol abused in conjunction with other drugs. This projection is based on national survey data of the U.S. population, the experience of employee assistance program representatives in Seattle-based industries, and the scient if ic literature, all of which suggest that multiple substance abuse among the employed are rapidly increasing. Thus, multiple substance abuse may also become a problem in the nuclear utility industry.

\section{Evaluation of Impairing Effects}

In order to evaluate the impairing effects of drug and alcohol abuse on nuclear power plant workers' job performance, a model of human performance is presented. The model includes four types of human performance: 
o Sensory/perceptual (inputting of information outside a person through the use of the senses)

- Cognitive (memory, information synthesis and decisionmaking)

o Motor (behavior--including movement, manipulating a dial, using a wrench, etc.)

o Communicative (verbal communications)

Next, the human performance model is related to five major job categories in nuclear power plants. The job categories (managers; scientists, engineers and other professional workers; licensed operators; non-licensed operators; and skilled craft workers) are taken from the 1982 Institute on Nuclear Power Operations (INPO) survey of nuclear-related employment. A judgment was made regarding the importance of the types of human performance to the five major job categories identified. The results of the analysis are presented in the following table.

Human Performance Requirements of Major Utility Jobs

\begin{tabular}{llllll}
\hline $\begin{array}{l}\text { Performance } \\
\text { Category }\end{array}$ & Managers & $\begin{array}{l}\text { Scientists } \\
\text { dEngineers }\end{array}$ & $\begin{array}{l}\text { Licensed } \\
\text { Operators }\end{array}$ & $\begin{array}{c}\text { Non-Licensed } \\
\text { Operators }\end{array}$ & $\begin{array}{l}\text { Craft } \\
\text { Workers }\end{array}$ \\
\hline $\begin{array}{l}\text { Sensory/ } \\
\text { Perceptual }\end{array}$ & Low & Medium & High & High & Medium \\
Cognitive & High & High & High & Medium & Medium \\
Motor & Low & Low & Medium & High & High \\
Commicative & High & Low & High & Medium & Low
\end{tabular}

\footnotetext{
Low $=$ Job category requires low frequency of doing tasks in specified performance area.

Medium = Job category requires medium frequency of doing tasks in specified performance area.

High = Job category requires high frequency of doing tasks in specified performance area.
} 
The impairing effects of drugs and alcohol on job performance were determined through an analys is of the scientific literature. Marijuana has its greatest effects on motor performance and cognitive performance and also affects sensory/perceptual performance, especially vigilance. Stimulants in small doses typically have a positive effect on performance. However, higher doses or chronic use can negatively affect all categories of performance. Sedatives, including minor tranquilizers and barbiturates, have a fairly wide-ranging and significant negative effect on all four human performance categories. Hallucinogens, although they are the least likely drug to be abused during work, also negatively affect all four areas of human performance. Finally, alcohol also negatively affects all four areas of human performance; however, the effects depend on the dose of alcohol. Low doses of alcohol affect sensory/perceptual performance and motor performance. Moderate doses of alcohol also affect cognitive and communicative performance.

The job performance requirements for the five occupational categories were then evaluated in light of the impairing effects analys is to determine which jobs would be most adversely affected by a given category of drugs or alcohol. Marjjuana was estimated to have the largest negative impact on the job performance of licensed and non-licensed operators, to have a medium negative impact on the performance of managers and skilled craft workers, and to have the smallest negative impact on the performance of scientists and engineers. Stimulants, if taken in small doses, are not likely to impact job performance negatively. Since alcohol and sedatives produce generally the same effects, both were estimated to have the largest negative impact on the performance of skilled craft workers, non-licensed operators, and licensed operators, in that order, and to have less negative impact on the performance of managers and scientists and engineers. Hallucinogens would have a large negative impact on the performance of all five jobs.

\section{Employee Assistance Programs}

Two different types of programs have been used in the past to minimize or eliminate drug and alcohol abuse in the workplace. The first type of program, which proved to be unsuccessful, tried to identify the drug addict or alcoholic based on symptomatology. These programs were not successful for at least four reasons. First, nobody above first-line supervisors was diagnosed as having a problem. Second, the program often looked like a witch hunt and was bad for employee morale. Third, drug and alcohol abusers were very skillful at diverting attention from their problem (drug addiction or alcoholism) to the reasons why they used drugs and alcohol. Fourth, because of the stigma that was attached to drug addiction and alcoholism, very few employees were correctly diagnosed.

In the $1960^{\prime} s$, the programs moved from symptomatology identification to being based only on job performance. The new programs were called employee assistance programs, because they were used to assist the troubled employee regardless of the reason for decrements in their job performance. The programs, therefore, were broader in coverage and were used to help employees with alcohol abuse problems, mental health and emotional problems, drug abuse problems, marital/family problems, and any other problems that 
negatively affected job performance. Because the programs are broadbrush in their approach, because they are only based on job performance, and because of the confidentiality assured by the program, employee assistance programs have been fully accepted by both management and labor. They have proven successful in returning $70 \%$ to $90 \%$ of troubled employees back to successful job performance. Thus, we suggest that the employee assistance programs approach be used in the nuclear utility program to overcome employee job performance decrements that are a result of any personal problem, including drug and alcohol abuse. 


\section{INTRODUCTION}

Drug and alcohol abuse among the general workforce of the United States has been recognized as a significant concern since the 1950's. Its major impact on industry has been in the areas of worker safety and worker productivity. National estimates that up to $47 \%$ of all non-fatal industrial accidents and $40 \%$ of fatal industrial accidents are alcohol-related attest to the impact of alcohol abuse on industrial safety records (National Inst itute of Alcohol Abuse and Alcoholism [NIAAA], 1978, 1981). In 1971, there was an estimated $\$ 9.35$ billion cost to industry from reduced product ivity among alcohol-troubled male workers, aged 21 to 59 . Aside from the higher rate of accidents, these workers are less efficient, have roughly five times the absentee rate of other employees, waste time while on the job, impair morale of co-workers, evidence faulty decisionmaking, require greater management time and attention, and experience higher rates of premature disability and death, resulting in the loss of employees in their prime who have skills that are difficult to replace (NIAAA, 1978, 1981). Similar patterns of reduced productivity have been reported among employees with drug abuse problems (Stephen \& Prentice, 1978). A widely-accepted, conservative rule of thumb used in industry to estimate the cost savings that can be realized by programs aimed at the drug and alcohol abusing employee is that these employees are about $25 \%$ less productive than other employees (Tuthill, 1982).

Smith (1982) provided estimates of the total workforce that could benefit from an employee assistance program for a variety of problems. In all, $10 \%$ to $12 \%$ of the workforce is estimated to need the services of an employee assistance program for behavioral problems (i.e., problems that affect job performance). Of the troubled employee group, Smith (1982) estimates that $45 \%$ to $50 \%$ suffer from alcohol abuse $(4.5 \%$ to $6.0 \%$ of the total workforce), $25 \%$ to $30 \%$ have a mental health or emotional problem (2.5\% to $3.6 \%$ of the total workforce), $13 \%$ to $15 \%$ have family problems (1.3\% to $1.8 \%$ of the total workforce), $7 \%$ to $10 \%$ suffer from drug abuse problems $(0.7 \%$ to $1.2 \%$ of the total workforce), and nearly $10 \%$ have other behavioral problems ( $1.0 \%$ to $1.2 \%$ of the total workforce). While this report is only concerned about drug and alcohol abuse, it is clear that there are other employee problems that can affect job performance that would benefit from the services provided by an employee assistance program.

Another perspective on the costs of employee drug and alcohol abuse to industry comes from programs set up to address these problems. Varjous organizations have calculated the savings that can be attributed to rehabilitation of the drug- and alcohol-abusing employee through company employee assistance programs. The Tennessee Valley Authority (TVA) estimates potential benefit of $\$ 215,097$ to $\$ 446,742$ attributable to the efforts of their Alcohol and Drug Dependency Program, a return of $\$ 2.73$ to $\$ 5.66$ per dollar spent on their program ("TVA Issues Annual Report," 1980). Levens (1976) estimates that in a typical company $11 \%$ to $21 \%$ of sick leave compensation, $11.5 \%$ to $54 \%$ of compensation for absenteeism, $11.5 \%$ of costs due to on-the-job accidents, $18.4 \%$ of health insurance 
costs, and $24.2 \%$ of the costs resulting from employee mortality can be attributed to employees abusing drugs and alcohol. Levens asserts that companies with employee assistance programs, where one-third of those seeking help for drug- and alcohol-related problems would be successfully treated, would realize a $3.8 \%$ to $7.3 \%$ cost savings for sick leave, a $3.8 \%$ to $7.8 \%$ cost savings for absenteeism, a $3.8 \%$ cost savings for on-the-job accidents, a $6.1 \%$ savings in health insurance costs, and a $8.1 \%$ savings in costs due to mortality. Since employee assistance programs report success rates of approximately $80 \%$ to $90 \%$ (Frey, 1983), Levens' estimates of typical cost savings to an organization appear to be conservative.

Aside from the direct evidence of the relationship between alcohol abuse and occupational safety (e.g., alcohol is involved in almost half of fatal and non-fatal accidents on the job), employee drug and alcohol abuse in the nuclear utility industry could also impact public health and safety from increased human error in plant operations. Increases in human error could be due to at least two causes--absenteeism and a direct effect on performance.

Employees who abuse drugs and alcohol are five times more likely to be absent from work than other employees. Workers who do not show up to perform their job must be replaced by someone else. The replacement pool generally comes from one of two sources: persons who are less familiar with the job (i.e., supervisors or temporary help), or persons who perform identical functions and are called on to work overtime or do double shifts. Both worker groups present potential safety liabilities: the former group because of their unfamiliarity with procedures and/or lack of recent experience, both of which are likely to increase errors and response time for the performance demands; and the latter group because of fatigue brought on by the long work hours. Also, as is discussed in detail in Sections 5. and 7., drugs and alcohol impair sensory/perceptual performance, cognitive performance, motor performance, and communicative performance. Such performance decrements imply increased human error, which could directly affect plant safety.

Over the past two years, the Nuclear Regulatory Commission (NRC) has shown a heightened awareness regarding drug and alcohol abuse problems in the nuclear utility industry and the implications for the safe operation of nuclear power plants. Recent drug incidents involving the arrest or termination of licensee or contractor employees are as follows: 1977, two; 1978, none; 1979, one; 1980, five; and 1981, twelve. By early 1982, at least four more terminations had been reported. The incidents

involved on-site possession and use of drugs, as well as personnel reporting to work under the influence of drugs (NRC, Information Notice No. 82-05). The drugs all come under the jurisdiction of the

Comprehensive Substances Act of 1970. All are classified under Schedules $I$ and II of the Act and, as such, are considered to have high potential for addiction (i.e., marijuana, hashish, amphetamines, phencyclidine, and methaqualone). Marijuana abuse was most frequently reported in NRC incident reports. The events included a variety of different employee groups, ranging from construction, operations, and security. 
Geographically, they occurred on a widespread basis, involving reactor sites in each of the five NRC regions.

In response to this information, an Office of Inspection and Enforcement (IE) Drug Abuse Task Force was established to address the problem on a generic basis. The Task Force surveyed a selected group of licensees and issued a report on the ir findings, "Survey of Industry and Government Programs to Combat Drug and Alcohol Abuse" (NUREG-0903). Additionally, two proposed rules to the Code of Federal Regulations (CFR), "Access Authorization Rule" (10 CFR 73.56) and "Fitness for Duty Rule" ( 10 CFR 50.54), are seen as an avenue by which a regulatory framework can be fashioned to guide utility programs that could address performance problems.

Some utilities have already undertaken an effort to address the need for employee assistance programs. Also, the Edison Electric Institute is currently assessing employee assistance programs in the industry. The industry is large and diverse. According to a recent survey conducted by the Institute of Nuclear Power Operations (INPO) of 58 of its member utilities, the nuclear industry respondents reported an employee population, excluding clerical and administrative staff, of approximately 26,099 on-site personnel (INP0, 1982). Thus, a variety of program strategies may be warranted.

Reliable information is not currently available on the nature, prevalence, and trends of orug and alcohol abuse among nuclear power plant workers. Because of the lack of this data and its importance to providing the NRC with guidance for possible regulatory action, this report uses estimates from the general population on the nature, prevalence, and trends of drug and alcohol abuse. Then, based on scientific literature regarding the effects of drug and alcohol consumption on job performance factors, the potential effects of drug and alcohol abuse on nuclear power plant worker performance are considered. Well-founded knowledge regarding the job categories most affected, the drugs most frequently abused on the job, and expected trends in drug and alcohol abuse are important to the formulation of employee assistance programs. Knowledge of other behavioral problems that negatively affect performance also needs to be considered. The assistance programs will benefit employees, protect public health and safety, and promote the productivity, efficiency, and safety of nuclear power plant operations.

The remainder of this report is divided into seven sections. Section 2 . summarizes the major findings of this report. The definitions of drug and alcohol abuse as employed in this report are discussed in Section 3 . In Section 4., the expected nature and prevalence of drug abuse are examined, focusing particularly on the employed U.S. population. Included here is a review of the scientific literature on drug abuse prevalence and patterns. In addition, findings from the results of the survey of local industries with established employee assistance programs are discussed. In Section 5., the impairing effects of drug abuse on job performance are considered, with attention to a model of human performance as it relates to job performance of the job categories 
relevant to the nuclear industry. The impairing effects examined are 1 imited to those resulting from drugs most typically abused in the general population. For the most part, the effects discussed are those arising from taking drugs on the job or immediately prior to reporting to work. In Section 6., the estimates of alcohol abuse prevalence in the general adult population and the U.S. workforce are presented. The findings from interviews with nuclear utility and local industry employee assistance program personnel concerning alcohol abuse are also presented in Section 6. Section 7. discusses the impairing effects of alcohol abuse on job performance. For the purposes of the impairing effects analysis, alcohol abuse primarily refers to drinking on the job or just prior to coming on shift. Performance decrenents due to chronic, off-the-job abuse are also discussed. Finally, Section 8 . presents the conclusions and recommendations of the report. 


\section{SUMMARY ANO CONCLUSIONS}

The original purposes of this report were twofold: (1) to characterize and describe the nature, prevalence, and trends of drug and alcohol abuse in the nuclear utility industry, and (2) to evaluate possible impacts of drug and alcohol abuse on decrements in job performance of nuclear power plant personnel. However, oniy minimal data specific to the nuclear utility industry are currently available regarding the first purpose. In addition, a direct survey of drug and alcohol abuse in the nuclear utility industry was not considered feasible. Thus, this report describes nature, prevalence, and trend issues as determined from non-nuclear industries and from the general, U.S. adult population. Without substantially more investigation, there is no way to determine whether employment practices within the nuclear utility industry would increase or decrease the prevalence of drug and alcohol abuse compared to the general population or non-nuclear industries. In order to provide a basis for regulatory planning, however, it is reasonable to assume that generally similar results would be found. Possible impacts of drug and alcohol abuse on nuclear-related job performance are identified based on currently available nuclear utility job descriptions and on the scientific literature regarding the impairing effects of drugs and alcohol on human performance.

The nature of the possible alcohol abuse problems is limited to the demographic characteristics of alcohol abusers and the problems caused by alcohol abuse, but not with the type of alcohol (e.g., beer, wine, and 1iquor) abused. The nature of the possible drug abuse problems deals with abuser demographics and types of drugs abused. Drug and alcohol abuse prevalence rates refer to the percentage of people who are abusing alcohol or specific drugs. Statements made about trends in the possible drug and alcohol abuse regard what is likely to be abused in the future. The possible effects of drug and alcohol abuse on job performance are drawn from medical and experimental research on the effects of drug and alcohol on specific performance.

Because minimal data are currently available, the estimates of the nature, prevalence, and trends of abuse were derived using several methodologies. First, the nature, prevalence, and trends for drug abuse and for alcohol abuse in the U.S. adult population were ident if ied through a survey of the scientific literature. These data were used as one way of estimating potential drug and alcohol abuse problems in the nuclear utility industry. Second, an expert in the area of employee assistance programs was consulted to identify established, successful employee assistance programs in the local business community. As a result, fourteen companies in the Seattle area that had over 1,000 employees were contacted and an interview was held with a representative of their employee assistance program in order to obtain estimates relevant to this study. These data were also discussed in this report as possible estimates for the nuclear utility industry, recognizing that the data are limited by the extent to wich regional influences in the Pacific Northwest may bias the data base. Finally, a sample of nuclear utilities was contacted by telephone in order to collect information from 
the nuclear utility industry. These data were compared to the estimates made from the other methodologies. The above data, combined with other information regarding employee problems, such as mental health and family marital problems, can then be used as a basis for the development of employee assistance programs in the nuclear utility industry.

\subsection{Drug Abuse}

Drug abusers were defined as those who use illegal drugs or use legal drugs illegally. National survey data indicate that those who are most likely to abuse drugs are: male, young adults, from large metropolitan areas, and from the Northeast region of the country. The drug most likely to be abused is marijuana. In 1979, approximately $35 \%$ of 18-25 year olds were current abusers (where current abuse is defined as using the drug at least once in the past month) and approximately $6 \%$ of those over 25 were current abusers of marjjuana. The next most abused drug is cocaine--approximately $9 \%$ of the 18-25 year olds were current abusers and 1\% of older adults were current abusers. Other drugs--such as stimulants, minor tranquilizers, sedatives, and analgesics ("pain killers")--are abused, but much less often.

Unfortunately, the survey data are not reported so as to make a specific statement about the percentage of all adults who currently abuse drugs. However, a range can be calculated--from $12 \%$ to $20 \%$ of all adults 18 years or older were current abusers of drugs in 1979. Other survey data from 1977 indicate that approximately 25\% of those who abuse drugs have done so at least once on the job. It is likely that on-the-job drug abuse remained at the same level or increased from 1977 to 1979. Combined with the above data, this would imply that from $3 \%$ to $5 \%$ (i.e., $25 \% \times 12 \%$ to $20 \%=3 \%$ to $5 \%$ ) of adults have abused a drug at least once on the job.

The survey data do not permit an estimate of the percentage of adults who have continual on-the-job drug abuse problems. However, it has been estimated by employee assistance program planners that approximateiy $1 \%$ of an average employee population shows significant decrements in job performance as a result of drug abuse. Representatives from the Seattle area employee assistance programs estimated that the most abused drug on the job is marijuana, followed by minor tranquilizers and then amphetamines and cocaine. They estimated that $4 \%$ of those seeking help from the employee assistance programs do so because of a significant drug abuse problem, but that actually $7 \%$ to $10 \%$ of those people that they see have a significant drug abuse problem. Depending upon the company, from $1 \%$ to $3 \%$ of employees had sought help from their company's employee assistance program. Therefore, in terms of actual employees seen by employee assistance programs who have a significant drug abuse problem, the maximum percentage would be $0.3 \%$ ( $j . e ., 10 \% \times 3 \%$ ) of the total workforce.

To summarize the above figures, an estimated $3 \%$ to $5 \%$ of U.S. adults have abused a drug at least once on the job, an estimated $1 \%$ of the U.S. workforce has significant on-the-job drug abuse problems so that employee assistance program help is needed, and an estimated $4 \%$ to $10 \%$ of 
employees who actually sought employee assistance program help in the Seattle area (which is less than $0.3 \%$ of all employees) did so for significant drug abuse problems. If it can be assumed that the nuclear power plant workforce is similar to the above populations, then these estimates also hoid for the nuclear power plant employees. However, because the nuclear power plant workforce is predominantly male and because men are more likely to abuse drugs than women, the above estimates may be underestimates when applied to the nuclear power plant workers.

\subsection{Alcohol Abuse}

Alcohol abusers are people who experience social, psychological, and/or physiological problems as a result of their alcohol intake. Thus, alcohol abusers include those who are true alcoholics (i.e., have the disease of alcoholism) and those who are not alcoholic but who do experience problems because of their drinking. Whether one is or is not an alcohol abuser can be determined through observable behaviors.

Survey data indicate that those most likely to have alcohol abuse problems are: male, 20-49 years of age, blue collar workers, and from large metropolitan areas. National surveys have found that approximately 11\% of the adult U.S. population are alcohol abusers. This $11 \%$ average is composed of approximately $4 \%$ of the adult female population and $17 \%$ of the adult male population. The following estimates of alcohol abuse for males for specific occupational categories are also available from national survey data: managers (18\%), scientists/engineers (10\%), licensed operators (10\%), non-1icensed operators (21\%), and skilled craft workers (21\%).

Depending on the company, from $45 \%$ to $100 \%$ of those employees who use the Seattle-area employee assistance programs do so for alcohol-related reasons. The employee assistance program representatives estimated that approximately $10 \%$ of their workforce suffered from alcohol abuse problems.

While the above estimates are not specific to on-the-job problems, it is realistic to assume that many alcohol abusers have on-the-job performance problems. Thus, alcohol abuse is likely to be much more prevalent among the nuclear power plant workers than is drug abuse.

\subsection{Multiple Substance Abuse}

A present and future problem in the U.S. industrial workforce is likely to be multiple substance abuse; that is, alcohol abused in conjunction with other drugs. This projection is based on national survey data of the U.S. population, the experience of employee assistance program representatives in Seattle-based industries, and the scient if ic literature, all of which suggest that multiple substance problems among the employed are rapidly increasing. Thus, multiple substance abuse may also become a problem in the nuclear utility industry. 


\subsection{Nuclear Utility Industry}

Eight representatives of nuclear utilities agreed to be interviewed over the telephone about drug and alcohol abuse at their plants out of 40 representatives who were contacted as part of the nuclear utility industry telephone survey. The representatives stated that they were aware of littie or no drug-related problems at their plants. Five of six representatives, who estimated alcohol abuse prevalence, estimated that less than $3 \%$ of the employees had alcohol abuse problems; the other representative estimated that the prevalence rate might be as high as 9\%. These responses suggest that the nuclear utility industry, like all other industries, recognizes that some alcohol abuse problems exist among its personnel. However, their estimates for alcohol abuse are much lower than the general population and general workforce estimates and the est imates provided by representatives from Seattle-area, non-nuclear industries. Regardless of the actual prevalence rates in the nuclear utility industry, the problems caused by drug and alcohol abuse in other industries and the usefulness of employee ass istance programs in overcoming these problems suggest that such programs are needed in the nuclear industry also.

\subsection{Evaluation of Impairing Effects}

In order to evaluate the impairing effects of drug and alcohol abuse on nuclear power plant workers' job performance, a model of human performance is presented. The model includes four types of human performance:

- Sensory/perceptual (inputting of information outside a person through the use of the senses)

- Cognitive (memory, information synthesis and decisonmaking)

- Motor (behavior--jncluding movement, manipulating a dial, using a wrench, etc)

- Communicative (verbal communications)

Next, the human performance model is related to five major job categories in nuclear power plants. The job categories (managers; scient ists, engineers and other professional workers; licensed operators; non-licensed operators; and skilled craft workers) are taken from the 1982 INPO survey of nuclear-related employment. A judgment was made regarding the importance of the types of human performance to the five major job categories identified. The results of the analys is are summarized in the following table.

The impairing effects of drugs and alcohol on job performance were determined. Marijuana has its greatest effects on motor performance and cognitive performance and also affects sensory/perceptual performance, especially vigilance. Stimulants in small doses typically have a positive effect on performance. However, higher doses or chronic use can negatively affect all categories of performance. Sedatives, including 
minor tranquilizers and barbiturates, have a fairly wide-ranging and significant negative effect. All four human performance categories are adversely affected by sedatives. Hallucinogens, although they are the least likely drug to be abused during work, also negatively affect all four areas of human performance. Finally, alcohol also negatively affects all four areas of human performance. Low doses of alcohol affect sensory/perceptual performance and motor performance. Moderate doses of alcohol also affect cognitive and communicative performance.

\begin{tabular}{|c|c|c|c|c|c|}
\hline & \multicolumn{4}{|c|}{ Drug Categories } & \multirow[b]{2}{*}{ Alcoho 1} \\
\hline & Marijuana & Stimulants & Sedatives & Hallucinogens & \\
\hline Managers & Medfin & $\begin{array}{l}\text { Monexistent } \\
\text { to Saali }\end{array}$ & Mediun & Larg̣e & Medium \\
\hline $\begin{array}{l}\text { Scient ists } \\
2 \text { Eng ineers }\end{array}$ & Small & $\begin{array}{l}\text { Monexistent } \\
\text { to } 5 \text { mall }\end{array}$ & Medila & Larqe & Medium \\
\hline $\begin{array}{l}\text { Licensed } \\
\text { Operators }\end{array}$ & Large & $\begin{array}{l}\text { Nonex istent } \\
\text { to Sall }\end{array}$ & Large & Larqe & Lorge \\
\hline $\begin{array}{l}\text { Mn-ticensed } \\
\text { Operators }\end{array}$ & Large & $\begin{array}{l}\text { Monex istent } \\
\text { to Small }\end{array}$ & Large & Large & Large \\
\hline $\begin{array}{l}\text { Skilled Crift } \\
\text { Uorkers }\end{array}$ & Meditas & $\begin{array}{l}\text { Nonexistent } \\
\text { to Sall }\end{array}$ & Lurge & Large & Large \\
\hline
\end{tabular}

Whefers to the effects of short-tern orug or alcohol use at low dose levels.

The job performance requirements for the five occupational categories were then evaluated in light of the impairing effects analys is to determine which jobs would be most adversely affected by a given category of drugs or alcohol. These effects are summarized in the table below.

Human Performance Requirements of Major vtility Jobs

\begin{tabular}{llllll}
\hline $\begin{array}{l}\text { Performance } \\
\text { Category }\end{array}$ & Managers & $\begin{array}{c}\text { Scientists } \\
\text { d Engineers }\end{array}$ & $\begin{array}{l}\text { Licensed } \\
\text { Operators }\end{array}$ & $\begin{array}{c}\text { Non-Lilled } \\
\text { Operators }\end{array}$ & $\begin{array}{l}\text { Craft } \\
\text { Horkers }\end{array}$ \\
\hline $\begin{array}{l}\text { Sensory/ } \\
\text { Perceptual }\end{array}$ & Low & Hedium & High & High & Medium \\
Cognitive & High & High & High & Medium & Medium \\
Motor & Low & Low & Medium & High & High \\
Communicative & High & Low & High & Medium & Low \\
\hline
\end{tabular}

\footnotetext{
Low * Job category requires low frequency of doing tasks in specified performance area.

Medium = Job category requires medium frequency of doing tasks in specified performance area.

High = Job category requires high frequency of doing tasks in specified performance area.
} 


\section{DEFINITION OF DRUG ABUSE AND ALCOHOL ABUSE}

Drug abuse is defined as the illegal use of a legal drug or use of an illegal drug. No consideration is given to the legitimate use of prescribed drugs. However, it is recognized that the psychoactive drugs discussed in this report can influence job performance under any circumstances. While the abuse of non-prescription drugs, which do not qualify as psychoactive drugs, has been noted as increasing, they are not included in this study because of absence of data regarding the ir use. Abuse of non-prescription drugs (e.g., over-the-counter diet pills and antihistamines) can, however, have an influence on human performance.

A number of definitions for alcohol abuse are available. For example, an early classic definition of alcoholism is derived from medical statistics on individuals who died because of cirrhosis of the liver due to alcoholism (Jellinek, 1960). Such a definition is quite conservative, because it only identifies drinkers for whom the habit had become life-threatening. A more commonly used definition identifies a person as suffering from alcoholism if their drinking is socially, physiologically, or psychologically debilitating (Sexias, Blume, Cloud, Leiber, \& Simpson, 1976). This latter definition has been used to identify populations for study, or treatment, as alcoholics.

For this study, we adopted a definition for alcohol abuse, rather than for alcoholism, which is based on observable drinking patterns and observable social, psychological, and physiological conditions of a person. This definition for alcohol abuse (also called problem drinking) was developed by Cahalan (Cahalan, 1978) and is based on the severity of problems in eleven categories of behavior. The eleven categories are:

- frequent intoxication--measured by the amount respondents Jrank on an occasion, the frequency with which they drank fairly large amounts, and their reports of how often they got "high" or "tight." A high score was attained on this variable if the respondent: drank five or more drinks at Jeast once a week; or drank eight or more drinks on one of the two most recent drinking occasions and twice in the last two months; or drank twelve or more drinks on one of the last two occasions and twice in the last year; or is currently getting high or tight at least once a week.

- binge drinking--consisted of being intoxicated for at least several days at a time or for two days on more than one occasion.

- symptomatic drinking--exhibition of signs attributed to physical dependence on alcohol and loss of control over drinking, including drinking to get rid of a hangover, having difficulty in stopping drinking, blackouts (lapses of memory), skipping meals while drinking, tossing down drinks for a quicker effect, and sneaking drinks. 
- psychological dependence--drinking to alleviate depression or nervousness or to escape from the problems of everyday living.

- problems with spouse or relative-included the spouse Teaving, threatening to leave, or becoming concerned over the respondent's drinking; the spouse or relative asking the respondent to cut down on his drinking; and the respondent judging his drinking as having had a harmful effect on his home life.

- problems with friends or neighbors--included the report that friends or neighbors had suggested that the respondent cut down on his drinking or that the respondent had felt that his drinking has been harmful to his friendships and social life.

- job problems--consisted of losing or nearly losing a job because of drinking, having coworkers suggest that the respondent cut down on drinking, and rating oneself as having harmed one's work opportunities through drinking.

- problems related with law and police--included reporting involvement with the law regarding driving while intoxicated or drinking contributing to an accident in which there was personal injury.

- health problems--based on reports that drinking had been harmful to the respondent's health and that his physician had advised him to cut down on his drinking.

- financial problems--based on reports that drinking had harmed the respondent's finances during the three previous years.

- belligerence--based on reports that drinking caused the respondent to feel aggressive or cross or was followed by a fight or heated argument.

Cahalan measured the eleven categories by using a total of 58 items. The number of items per category was unequal across categories, which generally had the effect of giving less weight to health problems, financial problems, and belligerence. If a respondent answered affirmatively to seven or more of the 58 items, Cahalan considered the respondent a problem drinker (alcohol abuser). It is important to note that the Marden formula, which is discussed in this report, is based on Cahalan's survey data of the drinking practices of the U.S. population. That is, Marden reanalyzed the Cahalan data and determined the percentage of respondents for a given sex, age, and occupation category who answered affirmatively to seven or more of the 58 items. 
Basing alcohol abuse on seven or more affirmative responses to the 58 items might seem overly conservative; however, Cahalan reported that:

- . a fairly high overall current problems score of seven points or more . . could be attained only by having problems in two or more areas, with at least one being rated as being severe in form; or problems in three or more areas, with at least two being at least moderate in severity; or problems in five or more areas, with at least one being moderate or severe; or slight problems in seven or more areas (Cahalan, 1970).

Also, of the eleven categories of alcohol-related problems, most are directly or indirectly related to job performance. For instance, frequent intoxication and/or binge orinking can cause lost days at work. In addition, because of the rate at which alcohol is broken down by the liver, a worker who drank heavily one evening could still have alcohol in his bloodstream at work the next day. If workers are experiencing symptomatic drinking, they are almost certainly experiencing job-related problems because they are likely to have alcohol in their bloodstream much of the time. Alcohol-caused problems with a worker's spouse, relatives, friends, or neighbors generally affect job performance.

We have therefore chosen to use Cahalan's definition of problem drinker (alcohol abuser) in this report for several reasons:

- The definition is based upon observable criteria that are indicative of social, psychological, and physiological problems related to the abuse of alcohol.

- Data from a national sample of adult respondents exist on which alcohol abuse prevalence rates have been calculated (the Marden formula).

- The Marden formula is now used by many state alcoholism agencies to estimate the number of alcohol abusers in their state as the basis of the statement of need for alcoholism treatment services.

- The definition is general enough to include people who are experiencing problems related to alcohol abuse, which often could affect job performance, but who have not yet become true alcoholics. 
4. NATURE, PREVALENCE, AND TRENDS OF DRUG ABUSE

IN EMPLOYED POPULATIONS

Problems associated with alcohol abuse were recognized before problems associated with drug abuse. Alcohol abuse has been a major concern to industry since the 1950's (Trice \& Roman, 1972). It was not until the "epidemic" of drug abuse in the late 1960's and early 1970's, however, that the abuse of drugs other than alcohol became sufficiently widespread in the workplace to require an industry response (Urban, 1973). As long as a decade ago, estimates of the proportion of companies with employee drug abuse problems ranged from $20 \%$ to $75 \%$ or even higher (Scher, 1973).

\subsection{Factors Inf luencing Drug Abuse Patterns}

An assessment of drug abuse practices among the employed population is complicated by several variables. First, there is a scarcity of information regarding on-the-job drug abuse. The most available source of information is from records of treatment intervention (i.e., treatment for acute reactions, long-term rehabilitation). However, on 7 y $20 \%$ of those treated for drug abuse have been employed (Stephen \& Prentice, 1978). Though there is a trend toward greater drug abuse in the employed population and drug abuse of greater severity, the intervention data, to date, do not provide good estimates for employed workers.

Geographic and demographic variables also influence the conclusions that can be drawn from general population data. Regional differences in drug abuse patterns occur as do differences related to population density. Fishburne, Abelson, and Cisin (1980) examined the drug abuse practices of a representative sample of the U.S. population. An analys is of the data for regular marijuana abusers indicated regional and population density differences and trends, as shown in Table 1 .

As can be seen from these data, the percentage of young adults and older adults who are regular marijuana abusers differs as a function of population density and region of the country. The most striking differences are between rural and metropolitan populations and between age groups. Abuse has increased significantly in the South for both age groups and in the North Central U.S. for young adults. All groups report greater abuse in 1979 .

Other demographic variables--sex, education, and occupation-also complicate the assessment of general and on-the-job drug abuse. Males report greater abuse of marijuana than females (with men also showing significant increase in abuse between 1977 and 1979). Among older adults, college-educated respondents were more likely to be current abusers (Fishburne et al., 1980). The data have recently been broken down by occupational groups for "older adults."* The occupational

\footnotetext{
*Fishburne, P., Response Analysis, personal communication between

P. Fishburne and L. Southwick, 1982.
} 
Table I

Current Marijuana Abuse Among Young (Age 18-25) and 0lder (Age 26+) Adults by Region and Population Density: 1976-1979a

\begin{tabular}{|c|c|c|c|c|c|c|c|c|}
\hline & \multicolumn{4}{|c|}{ Young Adults } & \multicolumn{4}{|c|}{ Older Adults } \\
\hline & 1976 & 1977 & 1979 & $\begin{array}{l}\text { Change: } \\
177 \text { 79* }\end{array}$ & 1976 & 1977 & 1979 & $\begin{array}{l}\text { Change: } \\
177-79 \star\end{array}$ \\
\hline \multicolumn{9}{|l|}{ Region } \\
\hline Northeast & $26 \%$ & $34 \%$ & $40 \%$ & NS & $5 \%$ & $5 \%$ & $7 \%$ & NS \\
\hline North Central & $27 \%$ & $29 \%$ & $38 \%$ & $S$ & $2 \%$ & $3 \%$ & $4 \%$ & NS \\
\hline South & $18 \%$ & $17 \%$ & $30 \%$ & SSS & $3 \%$ & $1 \%$ & $5 \%$ & SSS \\
\hline West & $35 \%$ & $33 \%$ & $36 \%$ & NS & $4 \%$ & $5 \%$ & $9 \%$ & NS \\
\hline \multicolumn{9}{|l|}{$\frac{\text { Population }}{\text { Density }}$} \\
\hline $\begin{array}{l}\text { Large } \\
\text { metropolitan }\end{array}$ & $29 \%$ & $31 \%$ & $39 \%$ & $S$ & $5 \%$ & $5 \%$ & $8 \%$ & NS \\
\hline $\begin{array}{l}\text { Sma11 } \\
\text { metropolitan }\end{array}$ & $28 \%$ & $29 \%$ & $36 \%$ & NS & $4 \%$ & $3 \%$ & $6 \%$ & $S$ \\
\hline $\begin{array}{l}\text { Non- } \\
\text { metropolitan }\end{array}$ & $16 \%$ & $18 \%$ & $30 \%$ & SSS & $1 \%$ & $1 \%$ & $4 \%$ & SS \\
\hline Rural & : & $:$ & $28 \%$ & -- & $!$ & $!$ & $3 \%$ & -- \\
\hline $2,500-24,999$ & : & $!$ & $37 \%$ & -- & $!$ & : & $4 \%$ & -- \\
\hline 2,499 or Jess & $!$ & ! & $23 \%$ & -- & $!$ & $!$ & $3 \%$ & -- \\
\hline
\end{tabular}

a Current abuse means that marijuana was used in the previous month.

* SSS = significant at .001 level (i.e., these data would be due to chance only one time in a thousand or less)

$S S=$ significant at .01 level

$S=$ significant at .05 level

NS = not significant

! Not tabulated. 
group that reported the highest level of current abuse was physicians $(25 \%)$, followed by technical workers (24\%) and sales workers (17\%). Engineers reported a moderate level of current abuse (10\%), and low levels of current abuse were reported by secretaries (1\%). Nurses, other health workers, and private household workers reported no marijuana abuse.

\subsection{General Population Survey Findings on Drug Abuse}

A major study of occupational drug abuse was conducted by Chambers and Inciardi (Chambers \& Inciardi, 1971a; 1971b) for the New York State Narcotic Addiction Control Commission. Approximately 7,400 in-person interviews assessed drug abuse in New York state in 1971. Projections based on employment status of participants suggested the following generalizations regarding the prevalence of drug abuse both on and off the job (Pradhan \& Dutta, 1977):

- The most commonly abused illicit drug is marijuana; $11 \%$ of the total sample reported at least one experience with marijuana, and a high proportion of regular abusers (27\%) reported some use during work hours.

- Regular abuse of non-barbiturate sedatives, antidepressants, and narcotics is uncommon (less than $1 \%$ of the population); regular abuse of barbiturates, prescription "pep" and "diet" pills, and minor tranquilizers is more common (about $2 \%$ of the population), and they are abused relatively frequently by sales workers.

- Regular abuse of the minor tranquilizers is highest among clerical and other white-collar workers ( $6 \%$ of this group), and if a worker is a regular abuser of either minor tranquilizers or prescription "pep" pills, there is a likelihood that at least some of this abuse occurs while on-the-job (15\% of regular abusers take these drugs while at work).

Results of the Fishburne et al. (1980) survey sponsored by the National Institute on Drug Abuse (NIDA) provided interview data from 7,224 members of the general population living in the contiguous United States.* Like the preceding survey by Chambers, results of this NIDA report indicated that--following alcohol--marijuana is the psychoactive substance that the public is most likely to abuse. In the 1979 survey (see Table 1), 68\% of young adults (ages 18-25) had used marijuana at least once in their lifetime (lifetime prevalence), and $35 \%$ had used marijuana within a month of the survey (past month prevalence). Among older adults (26 years or older), the lifetime prevalence rate was $20 \%$

* See also Table 1, above, for summary data concerning current marijuana abuse. 
and the past month prevalence rate was $6 \%$. The estimate for all adults combined is $30 \%$ for lifetime prevalence and $12 \%$ for past month prevalence (the $12.5 \%$ figures in Table 2 was rounded downward, because the calculated figure was $12.47 \%$ ). Of particular interest was a finding that among the subgroup of "older adults" aged 26 to 34 , who were young aduits during the early 70 's, the rates were considerably higher. of this group, almost $50 \%$ reported some experience with marijuana. Overall, Fishburne's et al. data for 1979 are substantially higher than the estimates based on Chambers' 1971 data. This suggests that there has been a significant increase in marijuana abuse among both young adults and older adults in recent years.

Other drugs examined in the 1979 NIDA study are listed in Table 2. They include hallucinogens, stimulants, sedatives, minor tranquilizers, inhalents, analgesics, heroin, and alcohol. Unfortunately, the data are not independent--i.e., someone who has abused marijuana might also have abused cocaine and/or alcohol and/or sedatives, etc. In conjunction with this, the authors state that abusers of other drugs are generally a subset of the marijuana abuser group.* This point will be discussed more later in this section. Other important points to be made from Table 2 (Fishburne et al., 1980) are:

- Compared to older adults, young adults have a higher lifet ime prevalence rate and past month prevalence rate for every type of drug.

- Next to marijuana, the most commonly abused illicit drug is cocaine. The age-weighted rate for all adults for lifetime prevalence is $11 \%$ and for past month prevalence is $3 \%$.

- Other drugs are abused at a much lower rate.

In order to estimate the prevalence rate for all drug abuse, two assumptions need to be made. First, we will assume that anybody who abused the drug in the past month regularly abuses the drug. Second, we need to make an assumption about independence of drug abuse. To do this we will look at two figures. First, we will assume that people who abuse a drug other than marijuana also abuse marijuana, so that our lowest est imate of drug abuse is simply the estimate of past month marijuana abuse. Second, for our highest estimate of drug abuse, we will assume that no person abused more than one drug. Thus, our highest estimate of drug abuse is found by adding together the past month prevalence rate for all drugs. This means that for young adults the drug abuse rate ranges from $35.4 \%$ (the percent of marijuana abusers) to $59.7 \%$ (the percent of marijuana abusers plus the percent of cocaine abusers plus the percent of all other drug abusers); for older adults, the drug abuse rate ranges from $6.0 \%$ to $7.9 \%$; and for tota 1 adults, the drug abuse rate ranges from $12.5 \%$ to $19.5 \%$.

«Fishburne, P., Response Analys is, personal communication, 1982. 


\section{Table 2}

Drug and Alcohol Lifetime Prevalence Rates and Past Month

Prevalence Rates for the U.S. Population in 1979

\begin{tabular}{|c|c|c|c|c|c|c|}
\hline \multirow[b]{2}{*}{ Type of Drug } & \multicolumn{2}{|c|}{ Young Adults $(18-25)^{a}$} & \multicolumn{2}{|c|}{ 01der Adults $(26+)^{b}$} & \multicolumn{2}{|c|}{ Total Adults $(18+)^{c}$} \\
\hline & $\begin{array}{l}\text { Lifet ime } \\
\text { Prevalence }\end{array}$ & $\begin{array}{l}\text { Past Month } \\
\text { Prevalence }\end{array}$ & $\begin{array}{l}\text { Lifetine } \\
\text { Prevalence }\end{array}$ & $\begin{array}{l}\text { Past Month } \\
\text { Prevalence }\end{array}$ & $\begin{array}{l}\text { Lifetime } \\
\text { Prevalence }\end{array}$ & $\begin{array}{l}\text { Past Month } \\
\text { Prevalence }\end{array}$ \\
\hline Marijuana & $68.2 \%$ & $35.4 \%$ & $19.6 \%$ & $6.0 \%$ & $30.3 \%$ & $12.5 \%$ \\
\hline Cocaine & 27.5 & 9.3 & 4.3 & 0.9 & 11.1 & 2.8 \\
\hline Hallucinogens & 25.1 & 4.4 & 4.5 & $\star$ & 9.0 & 1.0 \\
\hline Stimulants & 18.2 & 3.5 & 5.8 & 0.5 & 8.5 & 1.2 \\
\hline Sedatives & 17.0 & 2.8 & 3.5 & $\star$ & 6.5 & 0.6 \\
\hline $\begin{array}{l}\text { Minor } \\
\text { tranguilizers }\end{array}$ & 15.8 & 2.1 & 3.1 & * & 5.9 & 0.5 \\
\hline Inhalants & 16.5 & 1.2 & 3.9 & 0.5 & 6.7 & 0.7 \\
\hline Analgesics & 11.8 & 1.0 & 2.7 & $\star$ & 4.7 & 0.2 \\
\hline Heroin & 3.5 & $\star$ & 1.0 & $\star$ & 1.6 & - \\
\hline Alcohol & 95.3 & $75 . \subseteq$ & 91.5 & 61.3 & 92.3 & 64.5 \\
\hline
\end{tabular}

$a_{N}=2,044$.

$\mathrm{b}_{\mathrm{N}}=3,015$.

$\mathrm{C}_{\text {An }}$ age-weighted average based on 1980 census figures for the U.S. and assuming "older adults" ages ranged from 26-64. The assumption is that of all adults from 18 through 64 , $22 \%$ are from $18-25$ and $78 \%$ are from $26-64$.

*Less than $0.5 \%$. 
A further assumption is needed regarding the percentage of people who abuse drugs on the job. In a study by Chambers (1971) conducted in New York, $27 \%$ of the regular marjjuana abusers (which is comparable to the past month prevalence group for the present case) reported some use during working hours and $15 \%$ of the regular abusers of minor tranquilizers reported taking these drugs while at work. Since marijuana abuse accounts, by far, for most of the drug abuse, we will assume that $25 \%$ of drug abusers in general (i.e., drug unspecifjed) have abused their drug at least once at work (i.e., either took the drug on the job or took the drug prior to going to work so that they are still under the effect of the drug at work). Therefore, applied to the figures presented in the preceding paragraph, this means that: (a) from $8.9 \%$ to $14.9 \%$ of young adults have abused drugs on the job; (b) from $1.5 \%$ to $2.0 \%$ of older aduits have abused drugs on the job; and (c) from $3 \%$ to $5 \%$ of the total adult population have abused drugs on the job.

\subsection{Nuclear Licensee Survey Findings for Drug Abuse}

One of the major purposes of this study was to estimate the nature, prevalence, and trends of drug and alcohol abuse in the nuclear utility industry. Because no industry-specific data were available, we attempted to collect such data from nuclear litility representatives over the telephone. Forty licensees were selected to be included in the telephone survey. The plants were chosen from currently operating nuclear power plants with the following criteria in mind: (1) each utility with at least one operating plant would be included; and (2) for utilities with more than one operating plant, the most representative plant would be selected (considering number of units, size, type of plant, and plant age). Plant representatives were initially contacted by phone to identify the appropriate person on site to interview. The interview protocol used to conduct the interviews appears in Appendix A. Of the 40 plants contacted, partial interviews were completed with only eight iicensees. *

Despite the low response rate, some useful information was gained from the telephone discussions. Seven of the respondents' plants had an employee assistance program. These programs were staffed on a part-time basis. Most programs were two years old or less, and the majority were involved in performance evaluations--an approach typical of recentiy

* When it was clear that telephone contact with the utility representatives would yield little data, the sponsor was informed (11/4/82) and three strategies were outlined: (1) attempt to obtain industry support for the survey through contacts with industry groups;

(2) send a letter from the NRC requesting utility cooperation; and (3) emphasize obtaining relevant information from employee assistance programs in the Puget Sound area. All three strategies have been pursued. Two industry groups declined to provide any endorsement; however, information is expected to be shared from a similar survey undertaken by one of the groups. 
developed employee assistance programs (Wrich, 1980). In general, however, the utilities kept very little information on program use by employees. Therefore, respondents were unable to supply details of program use and types of problems experienced by employees. Thus, a large number of questions in the interview protocol typically went unanswered (e.g., Item $1(h)$ in Appendix A).

Likewise, many respondents were unable to estimate the extent of drug abuse in their plants. Half of the respondents indicated having seen no evidence of drug abuse. Two of them reported not knowing what the drug abuse problem might be. Two included drug abuse in their estimates of alcohol-related problems, both of which were estimated as less than $1 \%$ of the plant personnel. While four of the respondents could not estimate whether drug abuse had increased, decreased, or remained unchanged since 1977, three estimated that drug abuse had increased and one estimated that drug abuse had decreased in that period.

\subsection{Drug Abuse in Non-nuclear Industries}

As stated earlier, on-the-job drug abuse has been recognized as a problem in industry for at least 10 years. Estimates used as a basis for establishing employee assistance programs in industry are that $10 \%$ to $12 \%$ of any workforce will need a program for some reason, and approximately $1 \%$ need the program specifically for drug abuse problems (Smith, 1982).

In order to get additional statistics for purposes of this study on personnel needing assistance for drug abuse purposes, organizations with established and successful employee assistance programs were jdentified and contacted. The organizations were identified by an expert in the field of employee assistance programs. Representatives of employee assistance programs from these organizations in the Seattle area were interviewed regarding the extent and nature of employee drug abuse problems. An initial phone contact was made to set up in-person interviews conducted by one of three Battelle interviewers. One employee assistance progran representative refused to be interviewed due to a company policy prohibiting dissemination of information about employee drug and alcohol abuse. The fourteen organizations (see Appendix B) that were interviewed range in size from 1,500 to 100,000 employees, and most were engaged in some government-regulated work.

The longevity of the employee assistance programs surveyed varied widely. In general, the larger corporations have of fered employee assistance services the longest (e.g., one employee assistance program has been operating since 1945). Smaller organizations typically have initiated programs within the last five to seven years.

Placement of programs within the companies' organizational structures also differs. Some programs are in medical or human resource departments, while others are free-standing departments that report to upper management. None of the programs are part of personne] departments. In several organizations, outside contractors provide treatment services, while other corporations support in-house staff. 
Treatment services provided by the employee assistance programs range from evaluation and referrals only to comprehensive in-house programs, including long-term counselling, follow-up, and extensive management training and consultation.

Based on the interviews, changes have occurred in the nature of the employees' drug and alcohol abuse problems. The interviewees reported that the majority (70\% or more) of drug and alcohol abuse clients now present multiple drug and alcohol abuse problems, rather than just alcohol abuse problems. This is consistent with literature indicating a trend toward multiple drug abuse among the population in general (Carroll, Malloy, Hannigan, Santo, \& Kendrick, 1977).

An employee assistance program consultant in the Pacific Northwest, who works for five major corporations on the West Coast, explains this change in occupational drug and alcohol abuse in several ways. First, the youth of the 60's and early 70's drug culture have entered the workforce with their pre-existing drug abuse patterns and permissive attitudes towards drugs. Second, many of them began using drugs and alcohol at an earlier age than was common in the past, and so are developing abuse problems at an earlier age. Finally, more women are drinking now and using drugs more openty than was socially acceptable in the past.

According to the interviews, the types of drugs abused by employees also have changed in the past ten years. Use of marijuana on the job first became a problem in the early $70^{\prime} \mathrm{s}$, and marijuana has continued to be the primary drug of abuse to the present. For example, one respondent surveyed estimated that $70 \%$ of employees seen in their program smoke marijuana regularly. The representatives also reported that abuse of physician-prescribed minor tranquilizers (e.g., Valium (R) Librium (R)) is a companion issue to alcoholism in many cases and is frequent among alt employee groups.

Until 1979-1980, none of the employee assistance program personnel had been aware of a cocaine problem among employees. In the last two years, cocaine abuse on the job has increased in frequency. Individual habits are also becoming more serious, as evidenced by a concomitant increase in drug-related theft to support them. None of the representatives reported any cases of employee abuse of narcotics, such as heroin or opium.

Table 3 shows the most commonly abused drugs, ranked by the five employee assistance program representatives out of fourteen who had this information available. Because most of the drugs taken for recreational purposes are illegal, few employees feel safe in disclosing drug abuse problems, in contrast to problems with alcohol. Interviewees indicated that about $4 \%$ of the employees seeking assistance do so because of a significant drug abuse problem. However, they estimate that the incidence of drug abuse may range from $7 \%$ to as high as $10 \%$ of those seeking help. All of the employee assistance program personnel interviewed believed that the number of employee drug abuse problems is underestimated by their caseload statistics. In response to evidence of on-the-job drug abuse, one major organization formally expanded its 
Table 3

Most Frequently Abused Drugs by Employees on the Job, Ranked in Order of the Most Common Problem (1) to the Least (3)

\begin{tabular}{cccc}
\hline & \multicolumn{3}{c}{ Type of Drug } \\
\cline { 2 - 4 } Corporation & Marijuana & $\begin{array}{c}\text { Minor } \\
\text { Tranquilizers }\end{array}$ & $\begin{array}{c}\text { Aminhetamines } \\
\text { and Cocaine }\end{array}$ \\
\hline A & 1 & 3 & 2 \\
B & 2 & 1 & 3 \\
C & 2 & 1 & 3 \\
D & 2 & 1 & 3 \\
G & 1 & 3 & 2 \\
\hline Average Rank & 1.6 & 1.8 & 2.6 \\
\hline
\end{tabular}


program to include drug treatment three years ago. Representatives of several organizations attributed part of the increase to greater program emphas is on protecting confidentiality as well as increased incidence of drug abuse in the worker population.

\subsection{Summary and Conclusions}

General population surveys have found that marijuana is the drug most likely to be abused followed by cocaine and other central nervous system stimulants, central nervous system depressants (sedatives, minor tranquilizers, and analgesics), and hallucinogens. These findings were generally upheld by Seattle-area employee assistance program representatives, except for hallucinogens, which are rarely abused on the job.

General population prevalence estimates are that from $12 \%$ to $20 \%$ of adults over 18 are currently abusing one or more drugs. Approximately $3 \%$ to $5 \%$ of all adults have abused drugs on the job at least once. Employee assistance program consultants estimate that approximately $1 \%$ of a workforce needs assistance program help specifically for on-the-job performance problems related to drug abuse. Seattle-area assistance program representatives estimated that from $4 \%$ to $10 \%$ of those who sought help from their program had significant drug abuse problems. This translated to less than $0.3 \%$ of the workforce actually seeking help from the assistance program for a drug abuse problem.

A small sample of employee ass istance program representatives est imated that there were few to no on-the-job drug abusers at their plants. However, there have been approximately 24 drug-related incidents at nuclear power plants over the past five years. Thus, some on-the-job drug abuse has been detected.

Without corroborating estimates specific to the nuclear utility industry, a single estimate for drug abuse among nuclear utility industry personnel cannot be provided. The range of estimates, however, is suitable for making regulatory decisions regarding fitness for duty and access authorization. 


\section{IMPAIRING EFFECTS OF DRUG ABUSE ON JOB PERFORMANCE}

A major objective of this report is to examine the potential impairing effects of drug and alcohol abuse on job performance relevant to the nuclear industry. This section focuses on the effects of drug abuse on human performance. A link is made between human performance factors and job performance characteristics of the major job categories in the nuclear utility industry. Several groups of drug effects are presented, based on findings in the scientific literature.

Although numerous drugs were discussed in Section 4 ., only four drug classifications, which are based on overall drug effects, are used in this chapter. These are general central nervous system (CNS) stimulants, general CNS depressants (sedatives), hallucinogens, and marijuana. Though this four-group classification of drugs--stimulants, sedatives, hallucinogens, and marijuana--appears to omit many of the drugs discussed in the Executive Summary and Section 4., they can all be ident if ied according to these groupings. For instance, cocaine, a drug of increasing popularity, is generally classified as a stimulant, though it also has local anesthetic effects. Other stimulants are amphetamines, variously known as "speed," "diet," or "pep" pills. Sedatives include barbiturates, minor tranquilizers, and hypnotics (i.e., sleeping pills). Hallucinogens include LSD, mescaline and PCP.

As indicated in Section 4.4, marijuana, stimulants (in particular, cocaine), and sedatives (specifically, minor tranquilizers) are the drugs currently abused in the non-nuclear industries surveyed. A similar pattern (with the exception of inhalants) was found in the general population (see Table 2, Section 4.2).

The available data for on-the-job drug abuse show that hallucinogens are (among) the least frequently used $\operatorname{drug}(\mathrm{s})$ (Chambers, 1971). They were not identified as a major drug of concern among the non-nuclear employee assistance representatives interviewed (see Section 4.4). Hallucinogens, on the other hand, are anong the drugs reported in the NRC incidence reports (Information Notice No. 82-05); they are also the third most frequently used drug among the general population. A possible explanation for the contradiction is that hallucinogens are a popular drug of abuse but that their effects do not lend themselves to on-the-job abuse. Brief consideration will be given to them since they have been identified in incidence reports; however, hallucinogens are not expected to constitute a significant on-the-job drug problem.

\subsection{Model of Human Performance}

The purpose of this section is to discuss the impairing effects of various drugs on human performance, in general, and their potential effects on performance in five nuclear utility job categories. In order to do this, we must first present a model of human performance that can be used as a framework for the discussion. While several models of human performance exist, the model selected for use in this report needed to meet two criteria. First, the model needed to be relatively simple, 
since the research on the impairing effects on human performance of many drugs is scarce. Second, the model needed to be sensitive enough to allow some differentiation among the job categories.

The model that we have chosen is based upon a widely-accepted trichotomized categorization of human performance developed by Fitts and Posner (1967), which includes sensory/perceptual performance, cognitive performance, and motor performance. However, we shall follow the lead of other researchers (e.g., NUREG/CR-1750) and add a fourth category-communicative performance. In the three-category model, communicative performance would be subsumed under cognitive performance or motor performance. However, since communicating with others requires a social interaction not required by cognitive or motor performance and is also important for many job categories, we felt that it should be included as a separate category. Thus, our model includes four types of performance:

- Sensory/perceptual

- Cognitive

- Motor

- Communicative

Sensory/perceptual performance involves the inputting of information from outside a person through the use of the senses. Examples of sensory/perceptual performance include scanning control room displays for parameter deviations and listening for auditory warning signals. Cognitive performance involves internal information processing, including storage and retrieval of information in short-term and long-term memory, information synthesis, and decision making. Examples of cognitive performance are following procedures and determining the cause of an emergency event. Motor performance is simply behavior--e.g., using a wrench or manipulating a valve control pushbutton. Communicative performance is defined as communication of a verbal nature.

\subsection{Nuclear Utility Job Categories as Related to the Model of Human Performance}

The purpose of this part is to discuss how the four-category human performance model corresponds to five nuclear utility job categories 1isted in the "1982 INPO Survey of Nuclear Related Occupational Employment in U.S. Electric Utilities" (Institute of Nuclear Power operations, 1982). These five job categories, which are major job categories in nuclear power plants, include:

- Managers

- Scientists and engineers

- Licensed control room operators 
- Non-licensed control room operators

- Skilled craft workers (e.g., maintenance positions)

Each of these five job categories is discussed below.

\subsubsection{Managers}

Included in the job category "managers" are firstline supervisors through executives. The job requirements for managers include planning, organizing, directing, and/or controlling the activities of others (Campbe11, Dunnette, Lawler \& Weick, 1970; Labor Department Employment and Training Administration, 1977). Middle- to executive- level managers are involved primarily in activities requiring communicative (e.g., directing others, conferring with individuals) and cognitive (e.g., decisionmaking, planning, organizing) performance. Sensory/perceptual skills are needed to a lesser extent and only as aids to cognitive tasks. Within the category of managers, firstline supervisors also require communicative (e.g., training a new hire) and cognitive (e.g., analyzing work problems) skills. They may be involved more extensively in the activities of their subordinates than higher level managers and as such may be required to perform some sensory/perceptual and motor skills (e.g., a maintenance supervisor). However, the major characteristics of this job category are the communicative and cognitive skills. A sumary of the skills required of managers, as related to the four categories of human performance, is shown in Table 4 .

\subsubsection{Scientists and Engineers}

The second job category includes engineering positions and scientific positions (e.g., mathematician, computer scientist, chemist, physicist, biologist, and health physicist), as well as some technical and professional positions (e.g., chemistry technician, draftsman, quality assurance/control technician, and radiation protection technician). This category encompasses a wide range of jobs, each requiring some unique skills. However, it is possible to generalize across these jobs; job activities for both engineering and scientific positions together have been described (e.g., Danielson, 1960). One area especially critical for this job category is cognitive skills. These include conceptualizing, information synthesis, problem analys is, and decision making for planning/designing new or modified products or processes. To a lesser extent, sensory/perceptual performance (in conjunction with cognitive processes) is required of scientists. Motor skills are required only minimally, as they pertain to such things as the manipulation of equipment/instruments. Very little verbal comnunicative skill is essential for scientists except when discussing or reporting. A summary of the human performance skills for scientists is also shown in Table 4.

\subsubsection{Licensed Operators}

Several authors have made general statements about the major characteristics of the nuclear utility licensed control room operator's 
Table 4

Human Performance Requirements of

Major Utjlity Jobs

\begin{tabular}{llllll}
\hline & Managers & $\begin{array}{l}\text { Scientists } \\
\text { \& Engineers }\end{array}$ & $\begin{array}{l}\text { Licensed } \\
\text { Operators }\end{array}$ & $\begin{array}{c}\text { Non-Licensed } \\
\text { Operators }\end{array}$ & $\begin{array}{l}\text { Wraft } \\
\text { Workers }\end{array}$ \\
$\begin{array}{l}\text { Sensory/ } \\
\text { Perceptual } \\
\text { Cognitive }\end{array}$ & Low & Medium & High & High & Medium \\
Motor & High & High & High & Medium & Medium \\
Communicative & High & Low & Medium & High & High \\
\hline
\end{tabular}

Low = Job category requires low frequency of doing tasks in specified performance area.

Medium = Job category requires medium frequency of doing tasks in specjfied performance area.

High = Job category requires high frequency of doing tasks in specified performance area. 
job (this job category includes not only currently licensed operators, but also senior operators, and shift supervisors who hold senior operator 1 icenses). Jones and Eschenbrenner (1982) listed several general characteristics of the nuclear utility licensed operator's job. Five of these are discussed below.

The first characteristic is vigilance. That is, under routine operations at full power, for example, the operators spend long periods of time monitoring plant status from meters, recorders, displays, and readouts. Such monitoring requires sensory/perceptual skills and cognitive skills.

The second characteristic is decisionmaking, a cognitive skill. The operators must diagnose and correct plant disturbances using a myriad of inputs (e.g., annunciators, control panel displays, and safety parameter display systems), detailed knowledge about plant systems and system interactions, and operating policies and procedures.

A third characteristic of the licensed operator job deals with the distribution of the operator's physical and mental work load over time. As implied above, during routine, full power operations, this job can become quite routine. However, the operator can undergo periods of high physical and cognitive workloads, such as during planned outages and while responding to an emergency event. High cognitive workload, especially during the response to an emergency event, can be increased by the complexity of control/display panel layout, which frequently is not yet optimized for human operation.

A fourth characteristic of the operator's job is the need for teamwork through communications with others. An operator must communicate with other licensed operators, auxiliary operators, maintenance personne 1, and test and calibration personnel in order to coordinate activities. The licensed operator is one of the key communications people at the nuclear power plant.

The fifth characteristic is data use. That is, voluminous amounts of data, in the form of procedures, piping and instrumentation diagrams, computer-generated graphics and printouts, equipment diagrams, etc., are used in order to carry out his/her job. This activity requires cognitive performance.

Hodges' (1976) listing of operator (licensed) tasks includes some of the Jones and Eschenbrenner (1982) characteristics, but it also includes other task descriptions that are relevant to this discussion. First, Hodges (1976) notes that generally an operator's work time is filled with routine and repetitive tasks that, taken in isolation, are not difficult to perform. Much of what an operator does is follow procedures (rule-based cognitive behavior). In order to follow the procedures, the operator must know how to use them correctly, be able to carry them out, and be willing to use them. While much of the operator's tasks are routine, difficulties in performance arise when the operator must perform near simultaneous communications actions, troubleshooting actions, control actions designed to keep the reactor in a safe state, and data 
collection actions. Again, these actions are required during times such as emergency events, and they involve all four types of human performance (sensory/perceptual, cognitive, communicative, and motor). In this case, the operator is required to carry out all four types of functions nearly simult aneously.

Hodges (1976) also makes the point that the operator's job entails "coordinated manipulative tasks." These tasks involve, for example, frequent set point adjustments of automatic controls and manual control of valves, pumps, etc., during startups and shutdowns. This requires mainly perceptual and motor performance, although some cognitive performance is also needed.

Finally, Hodges (1976) maintains that an operator must operate from a large knowledge base. That is, the operator must be ". . a bit of a physicist, nuclear engineer, instrument engineer, and water chemist" (p. 7). This adds to the operator's cognitive requirements.

To summarize, the licensed operator's job involves a significant reliance on sensory/perceptual and cognitive skills. Recognizing that communicative and motor skills also are key to the satisfactory performance of their job, operators are heavily reliant on the ability to scan instruments, sustain vigilance, follow procedures, diagnose and correct plant disturbances and draw on a broad data and knowledge base. Our assessment of the skills required of licensed operators as they pertain to the four-factor model of human performance is shown in Table 4 .

\subsubsection{Non- ] icensed Oper ators}

The non-licensed operator job requires many of the same types of skills as the Jicensed operator job, but generally to a lesser extent. The non-licensed operator must perform sensory/perceptual tasks (e.g., monitor plant status from displays), cognitive tasks to a certain degree (the licensed operator usually plays a greater role in decision making), motor tasks perhaps even more than the licensed operator (e.g., performing motor behaviors such as under the direction of a licensed operator), and communicative tasks somewhat (e.g., coordinating with other operators under the direction of a licensed operator). The required skills are shown in Table 4.

\subsubsection{Skilled Craft Workers}

Skilled craft workers is another job category used in the INPO survey. Nuclear utility employees who would fit into this category include technical and maintenance individuals, in general, and people such as nuclear utility instrument technicians, in particular. These positions would be in the areas of maintenance, repair, fuel and materials handling, and typically require extensive training and/or apprenticeship programs.

An example of a specific skilled craft workers job in a nuclear utility is the job of instrument technician. The performance requirements of 
this job, as described in the "Dictionary of Occupational Titles" (Labor Department Employment and Training Administration, 1977), include:

- Inspects, tests, adjusts, and repairs electric, electronic, mechanical, and pneumatic instruments and systems. . .

- Removes defective instruments from system . . and replaces defective parts using handtools.

- Calibrates readings on instruments according to standards and adjusts phasing and aligns stages to insure accuracy of recording. . . .

- Prepares schematic drawings, sketches, and reports to reflect changes or alternations made. . . .

While the requirements of skilled craft workers' jobs no doubt differ depending on department and utility, there are some general requirements of individuals in these positions. These jobs often require the use of tools and/or equipment. This necessitates some sensory/perceptual skills to correctly go about doing the task. Moreover, motor skills are needed to handle the tools/equipment. Cognitive skills would be required especialiy when diagnosis of a problem is necessary. Communicative skills would be necessary only to the extent of discussing a problem with others. A summary of the job requirements of skilled craft workers, as related to the four-factor model of human performance, is also shown in Table 4.

The following sections will briefly summarize the impairing effects and the major drug categories identified in Section 4.6. A more in-depth review of the impairing effects of these drugs is found in Appendix $D$.

\subsection{Marijuana}

As the most commonly used drug, other than alcohol, the effects of marijuana on human performance have received increasing attention in the scientific literature over the past ten years. Delta-9-

tetrahydrocannibol (THC) is the active ingredient in marijuana and in hashish, a marijuana-derivative made from resins in the female flowers of the Indian hemp plant, Cannabis sativa. Ingestion or inhalation of THC increases pulse rates and dilates the blood vessels in the eyes. Absorption from the lungs is rapid and complete, and peak blood concentrations occur 10 to 30 minutes after smoking a marijuana cigarette. Effects of the drug last typically for 2 to 3 hours (though some effects have been noted for up to $41 / 2$ hours), but excretion of marijuana metabolites may require a week or longer. Marijuana use is not physiologically addicting, despite its legal classification as an addicting drug under the Controlled Substances Act. Some users have reported a psychological dependence on this drug, however, and tolerance to its psychological and physiological effects develops with repeated use. 
Though the findings of the scientific literature are not entirely consistent, marijuana influences an individual's ability to perform a number of sensory/perceptual skills. Decreased hearing ability as well as a decreased ability to either recognize or process visual stimuli is reported. Also, evidence points to a reduced ability to sustain attention in a vigilance task.

Marijuana intoxication is associated with significant motor performance decrements on a variety of reaction time, motor steadiness, manual dexterity, and eye-hand coordination tasks. In some instances these decrements continue for as long as four and one-half hours after ingestion of the marijuana. Practice of the motor skills involved in reaction time for complex tasks may reduce performance decrements.

The effects of marijuana on cognitive tasks are not readily understood. In general, however, marijuana intoxication affects memory when the material to be recalled is learned in an intoxicated state. Numerica] reasoning is significantly impaired; other forms of reasoning were not consistently affected by marijuana intoxication. No data are available on marijuana intoxication and decisionmaking. Several studies of interpersonal judgment suggest that social behavior is impaired by marijuana intoxication.

The effects of marijuana on communication performance have not been studied. Communication, however, can be expected to be influenced to the extent that complex reaction time, memory, numerical reasoning, and social judgment are involved in the creation of specific verbal behavior. Chronic (i.e., long-term and frequent) abuse of marijuana does not appear to lead to the physical and psychological deterioration associated with chronic abuse of other drugs (e.g., stimulants, sedatives, and alcohol).

Studies of the effects of marijuana on performance when combined with other drugs have been limited to the combination of marijuana and alcohol. These studies have consistently shown that the effects of these two drugs are additive, so that any impairment found for either drug will also be found when both drugs are taken together.

\subsection{St imulants}

The class of stimulant orugs includes the amphetamine family and cocaine. The amphetamines and cocaine are functionally similar. The major functional difference is one of intensity and duration of effect.

The amphetamines have few legitimate medical uses. They are rarely prescribed for treatment of narcolepsy and petit mal epilepsy. Amphetamines are also prescribed for appetite suppression. However, the effect for a constant daily dose lasts only about two weeks, and appetite returns to normal unless the amphetamine dose is increased. Cocaine is used medically for local anesthesia and shrinkage of mucous membranes in surgery, although newer forms of anesthesia are typically substituted. 
The effect of stimulants on human performance has been evaluated in the scientific literature; however, none of the experimental work reviewed was done with cocaine. Though there are few differences between cocaine and amphetamines, the conclusions drawn regarding performance effects of stimulants may differ from performance effects resulting from cocaine abuse.

Vigilance performance, or the ability to attend to sensory input, has been improved in fatigued subjects given low doses of amphetamines. Motor performance is also affected by stimulants. Ath letic performance (swiming, running, etc.) is improved significantly in nonfatigued subjects given doses of amphetamines. Tests of reaction time show fatigued and nonfatigued subjects react more rapidiy on a variety of simple and complex tasks. The only adverse motor response seems to be evidence of fine tremors in unfatigued subjects.

The effect of stimulants on cognitive performance is less straightforward. In general, simple cognitive performance has been noted to improve in subjects given stimulants. Limited evidence exists to indicate that more complex cognitive performances are unaffected by stimulants. No enhancement of short-term memory skills has been found and amphetamines have been found to have no effect on performance tests requiring higher level cognitive functioning such as is used in calculus. Most of the positive effects of amphetamines on cognitive performance are generally attributed to the drug's ability to enhance attention and alleviate boredom on simpler repetitive tasks rather than enhance thinking and judgment in complex cognitive tasks. Stimulants increase the amount of talking in a social situation.

The effects of chronic use of stimulants are several. Tolerance develops quickly (approximately two to four weeks if used daily) and therefore increasing doses must be administered to achieve the same behavioral effects. Tolerance is thought not to occur, however, with cocajne ( $V$ an Dyke \& Byck, 1982). Sleep disturbances occur with stimulant abuse; prolonged abuse also leads to physical damage, massive depression when the effects wear off (suggestive of physiological withdrawal), compulsive repetitive behavior and paranoid psychosis that is indistinguishable from naturally occurring psychotic disorders.

\subsection{Sedatives}

The sedatives are the most commonly physician-prescribed drugs, and although they differ somewhat in chemical composition and site of action in the body, all drugs in this class are central nervous system depressants. Familiar sedatives are the barbiturates, the anxiolytics or minor tranquilizers, ethanol, and the general anaesthetics. At low doses, all of these drugs are capable of producing behavioral disinhibition and euphoria. At higher doses, each of them produces drowsiness, and can induce unconsciousness if taken in sufficient amounts (Julien, 1981). 
The drugs in this class which are most likely to be used by employed individuals are the minor tranquilizers, the barbiturates, and alcohol. The effects of alcohol on human performance are discussed in Section 7 . The action and effects of the minor tranquilizers and the barbiturates are described below.

\subsubsection{Anxiolytics}

Included in the minor tranquilizers are the benzodiazepines and the dicarbamate derivatives. There are over 2,000 benzodiazepine compounds; the ones most commonly prescribed are diazepam ("Valium" (R)), chlordiazepoxide ("Librium" (B)), and flurazepam ("Dalmane"(B)). Among the dicarbamates, meprobamate ("Miltown" (B) or "Equanil" (B)) is the most widely prescribed. For the purposes of the following discussion, all of these will be referred to as minor tranquilizers or by commonly known brand names. These drugs are effective in the treatment of tension, anxiety, and psychosomatic disorders. They are also used for treatment of alcoholism and phobic states (Gaston \& walker, 1981). Valium $\mathbb{R}$ is prescribed for symptomatic relief of anxiety and tension, alleviation of the symptoms of acute alcohol withdrawal, relief of muscle spasms, treatment of convulsive disorders, alleviation of pre-surgical anxiety, and as a hypnotic (sleeping pi11).

The effects of the minor tranquilizers on sensory functioning have not been extensively investigated. Findings suggest that minor tranquilizers produce some visual and auditory disruptions. Vigilance performance is impaired to a greater extent than visual and auditory performance. When minor tranquilizers are chronically used at low doses, no changes in either speed of reaction or number of response errors have been found. Standard or low doses do not appear to significantly impair most motor performance. Speed of physical movement is not affected by low doses of minor tranquilizers. Tests of eye-hand coordination have also shown no minor tranquilizer-induced performance decrement.

Significant valium (B) effects on simulated driving performance, however, have been found. Drugged subjects drove faster, neglected instructions more frequently, and caused more collisions than did subjects given a placebo, but evidenced no decrease in their ability to stay on the road.

Minor tranquilizers appear to have their greatest effects on learning and, to some extent, memory. Substantial evidence exists to suggest that tranquilizers impair learning, but do not interfere with the ability to retrieve information from memory once it has been stored.

\subsubsection{Barbiturates}

Although barbiturates and minor tranquilizers are chemically dissimilar, most of the effects of typically prescribed doses of barbiturates on human performance are highly similar to the effects of larger doses of minor tranquilizers. The primary medical uses of barbiturates are to produce sedation or sieep and to prevent epileptic seizures. At typical doses, the barbiturates depress the transmission of nerve impulses across 
the synapses in the arousal centers of the brain. At larger doses, all neurons in the body are affected and activity in the muscles, heart, and other organs of the body is decreased.

Barbiturates impair the ability to sustain attention. Results of several studies indicate that the number of errors subjects make on vigilance tasks is increased when they are drugged. Unlike the minor tranquilizers, typical doses of barbiturates slow reaction times. As found with the minor tranquilizers, the effects of barbiturates on psychomotor performance depend upon the dose. At the dose levels prescribed by physicians, simple motor performance is not noticeably affected (e.g., finger or toe tapping), but is impaired when the dose level is increased. More complex tests of psychomotor performance, however, show barbiturate induced performance decrements at therapeut ic dose levels. Performance on tests of eye-hand coordination is impaired with barbiturates.

Studies of the effects of barbiturates on cognitive abilities indicate that the barbiturates have a much greater effect on cognitive abilities at typical dose levels than do the minor tranquilizers. Memory, learning, and reasoning abilities have all been shown to be impaired by barbiturate use. Barbjturates also affect communicative skills. Persons who have been given typical doses of barbiturates both speak less in a social situation and speak slower than persons given a placebo.

\subsubsection{Interactive Effects of Sedatives}

To examine the interactive effects of sedatives, some further discussion of the effects of sedatives on the central nervous system is useful. Their effects are dose-dependent, with a progression with increasing dose as follows: anxiety reduction $\rightarrow$ disinhibition $\rightarrow$ sedation $\rightarrow$ hypnosis (sleep) $\rightarrow$ general anesthesia $\rightarrow$ coma $\rightarrow$ death (Julien, 1981). They are most effective when their use is time-limited (i.e., used for 1 to 8 weeks and then stopped until symptoms recur) (Julien, 1981). Nonetheless, it has been estimated that two milition persons in the United States take Valium (B) continuously (Jick, 1974, cited in Linnoila, 1976).

When combined with other central nervous system depressants such as alcohol or other drugs within the class of sedatives, sedatives are referred to as having "potentiating" effects rather than being simply additive as with marijuana and alcohol. The significance of the potentiating interaction of sedatives has greatest bearing on multiple substance abuse. For example, the individual who has a standard dose of a sedative and then a drink or two at lunch will experience an effect that is greater than the simple addition of one unit of effect due to the sedative, plus two units of effect due to the alcohol. The unpredictability of one sedative in particular, Valium $(\mathrm{R}$ has been noted; 
the interactive effects are so unpredictable that the combination of one drink and one standard dose has been known to cause death. ${ }^{\star}$ )

Performance decrements due to sedative interaction effects have not been covered in the scientific literature. It is likely, however, that the effects on performance noted above would become more severe when two different sedatives are taken together. Also, the course of events outlined above clearly suggests that attention and learning would be significantly impaired with increasing doses of sedatives as individuals progress toward a hypnotic condition.

\section{5 .4 Sumbary}

To sumarize, sedatives when taken at standard dose levels are found to produce the following effects:

- Performance decrements due to sedative ingestion have been found for perceptual/sensory tasks; auditory and visual capacity is less sensitive to input; however, the most pronounced effect found is for performance decrements in sustaining attention and concentrating on tasks requiring vigilance.

- Some types of motor performance are affected by sedatives (i.e., choice reaction time) but performance decrements disappear when sedatives are chronically used; other motor skills such as eye-hand coordination, complex driving tasks, etc., show no decrements due to low doses of minor tranquilizers, but are impaired by barbiturates.

- Cognitive performance, in particular the learning of new material, is impaired; when learning occurs in a sedated state, subjects show significant impairment in their ability to evidence the learning and to retrieve or recall the information at a later point in time.

- Communicative skills are not likely to be adversely affected by low doses of minor tranquilizers, but are impaired due to barbiturates.

\subsection{Hallucinogens}

Hallucinogens consist of a variety of drugs that distort sensory perceptions, thought processes, and behavior. It is thought that these drugs operate on, and highly resemble, naturally occurring chemicals in the brain (Julien, 1981). It is expected that this class of drugs will be used on the job only rarely; consequently, the review of their effects on performance is limited in scope and detaji.

\footnotetext{
*Rose, Mitchell. Lecture given for Association for Advanced Training in the Behavioral Sciences, Los Angeles, California, March 1982.
} 
Four groups of hallucinogens have been identified on the bas is of their effect on particular chemical substances within the brain. The first of these, the "anticholinergics" (e.g., atropine, scopolamine, malathion) are highly toxic and produce severe side effects. Many insecticides such as malathion are rarely used except by seriously debilitated drug abusers. Consequently, they are not addressed here, since few of the abusers are likely to be employed.

The second group of hallucinogens, the catechols, includes peyote, mescaline, and synthetic mescaline agents such as MDA and MMDA. The most pronounced effects associated with the use of these substances are the marked distortions in perception of light, color, space, and shapes. Users are typically alert and give no evidence of memory loss. Ingestion of large doses (i.e., 200 to $500 \mathrm{mg}$. for mescaline), however, may lead to severe muscle spasms (Julien, 1981).

Third, the indole group consists of LSD, psilocybin, and morning glory seeds (ololjuquj). While users seldom experience serious physical effects, they frequently encounter dramatic changes in mood and sensory perception (Gaston \& Walker, 1981). Actual effects are apt to vary, depending on the user's expectations, amount of drug ingested, personality, and setting. In general, effects include an altered sense of time, space, touch, color, and blurred vision and hearing. With higher doses (i.e., 5 to $15 \mathrm{mg}$. for psilocybin and 200 to $500 \mathrm{mg}$. for LSO), one experiences mood fluctuations (euphoria, fear, hostility), problems in speaking clearly, visual hallucinations, confusion, and impairment of thought processes. A decline in motor function and increased sleepiness are also linked with psilocybin use. Although rare and unpredictable, flashbacks may occur up to one year after use of LSD.

Finally, use of the hallucinatory anaesthetics (the most common being $P C P)$ is linked with general confusion, spatial disorientation, aggressiveness, and feelings that one has great physical strength or that one is about to die (Gaston \& Walker, 1981). The user may experience trouble breathing and, at higher doses, become unconscious. There is, in addition, little sense of pain. Although long-term and behavioral effects are quite unpredictable, the anaesthetics are considered to be the most dangerous of hallucinogens, possibly resulting in permanent brain damage.

Dverall, the occasional use of hallucinogens has not been shown to result in physical addiction, chromosomal damage, anti-social behavior, or long-term psychological disorientation (Gaston \& Walker, 1981). Use of hallucinogens on the job, however, presents a severe safety danger and would preclude effective job performance for any employee.

\subsection{Effect on the Nuclear Industry}

The effects of the drugs reviewed in the preceding sections $(5.3,5.4$, 5.5 , and 5.6) on the four factor model of human performance will be summarized in this section. These effects will then be related to the rating of each of the performance factors for the five major job 
categories. Implications will be drawn for possible effects on personnel in the nuclear industry.

The impairing effects of marijuana on human performance have greatest impact on motor skills (slowed reaction time, reduced motor steadiness and manual dexterity) and aspects of cognitive functioning (impaired ability to recall material learned while intoxicated, impaired numerical reasoning, and impaired social judgment). Other performance categories affected include sensory/perceptual as marijuana reduces vigilance capabilities.

The effects of marijuana last for two to three hours after intake; for some measures of motor performance decrements have been noted for as long as four and one-half hours. Though no effects have been noted for chronic use of marijuana, the effects for any given social dose, if ingested or smoked prior to work, could clearly last long enough to produce on-the-job performance decrements.

Stimulants have a generally positive effect on human performance, particularly motor performance in fatigued subjects. The real risks from stimulants occur from chronic abuse. The performance effects of chronic sleep loss, followed by periodic "crashes," are obvious in terms of employee productivity and quality of work.

Sedatives, both minor tranquilizers and barbiturates, have fairly wide-ranging effects. All four human performance categories are negatively affected. The sensory/perceptual abilities affected by sedatives are visual acuity, the ability to track moving objects, the ability to sustain vigilance, and the ability to identify or detect signals. Furthermore, barbiturates cause a tendency to react to signals that do not exist. The primary motor skills that are affected are reaction time, which lengthens, and eye-hand coordination, which is reduced. Cognitive ability is significantly affected, particularly by barbiturates. Short-term memory ability is generally impaired; learning is affected by the inability to store new information (and do it accurately). Finally, problem-solving ability is impaired. Communication skills are also affected as rate of speech is reduced significantly by the use of sedatives.

Although hallucinogens are abused, they are not expected to be a significant problem for on-the-job use at nuclear power plants. They have substantial negative effects on sensory/perceptual, motor, and cognitive processes. No studies were available regarding their effect on communciation behavior, though it is likely that disorientation, confusion, and visual hallucinations occurring would also render communication abilities very impaired.

In sumnary, the following impairments can be expected:

- Marijuana will impair motor and cogitive performance with a lesser influence on sensory/perceptual performance. 
- Stimulants will increase motor and cognitive performance; they will impair performance if chronically abused.

- Sedatives will impair all areas of human performance.

- Hallucinogens will impair all areas of human performance.

Which job categories will these drugs negatively affect? Before briefly summarizing this information, it is important to reconsider what percentage of power plant personnel might be affected by drug abuse. The est imates from Section 4 . were that approximately $12 \%$ to $20 \%$ of the adult public are current drug abusers, approximately $3 \%$ to $5 \%$ of the adult public has abused a drug on the job at least once, approximately $1 \%$ of the workforce is significantly impacted by drug abuse so as to negatively affect performance, and approximately $0.3 \%$ or less of the Seattle-area workforce covered by employee assistance programs use such programs for drug abuse problems.

Table 5 summarizes the estimated negative impacts from the impairing effects of the four drug categories on the five occupational categories relevant to the nuclear utility industry. These are global summary judgments based upon the performance requirements for the occupationa] categories and on the impairing effects of the drugs on the categories of performance.

As can be seen from the table, marijuana is expected to have a small negative impact on the job performance of scientists and engineers, a medium negative impact on managers and skilled craft workers, and a large negative impact on licensed operators and non-licensed operators. Stimulants are expected to have a nonexistent to small negative impact on all job categories if used in small doses. Sedatives are expected to have a medium negative impact on the job performance of managers and of scientists and engineers and a large negative impact on licensed operators, non-licensed operators, and skilled craft workers. Hallucinogens are expected to have a large negative impact on job performance in all five occupational categories. 
Table 5

Est imated Impact of the Impairing Effects of Four Types of Drugs on Job Performance for Five Occupational Categories ${ }^{a}$

\begin{tabular}{lcccc}
\hline & & \multicolumn{2}{c}{ Drug Categories } & \\
\cline { 2 - 5 } & Marijuana & Stimulants & Sedatives & Hallucinogens \\
\hline Managers & Medium & $\begin{array}{l}\text { Nonexistent } \\
\text { to Small }\end{array}$ & Medium & Large \\
$\begin{array}{l}\text { Scientists } \\
\text { \& Engineers }\end{array}$ & Small & $\begin{array}{l}\text { Nonexistent } \\
\text { to Small }\end{array}$ & Medium & Large \\
$\begin{array}{l}\text { Licensed } \\
\text { Operators }\end{array}$ & Large & $\begin{array}{l}\text { Nonexistent } \\
\text { to Small }\end{array}$ & Large & Large \\
$\begin{array}{l}\text { Non-licensed } \\
\text { Operators }\end{array}$ & Large & $\begin{array}{l}\text { Nonexistent } \\
\text { to Small }\end{array}$ & Large & Large \\
Skilled Craft & Medium & $\begin{array}{l}\text { Nonexistent } \\
\text { to Small }\end{array}$ & Large & Large \\
\hline
\end{tabular}

aRefers to the effects of short-term drug use at low dose levels. 
6. NATURE, PREVALENCE, AND TRENDS OF ALCOHOL ABUSE

IN EMPLOYED POPULATIONS

Alcohol abuse prevalence can be established by several techniques. One approach is to base the estimates on the incidence of alcoholism in the general population. A second approach is to survey a population similar to the group of interest and use the results as an indicator of expected rates. A third approach is to sample a specific population directly to determine the prevalence. This last technique is preferred, since it increases the accuracy of expected rates. All three approaches were used in this research. The results are presented in the following sections.

\subsection{Factors Influencing Alcohol Abuse Patterns}

As is the case for drug abuse, alcohol abuse prevalence is related to several demographic variables. The relationship of alcohol abuse to the most important demographic variables is briefly discussed in this section. These data are taken from Cahalan et al. (1969) and are therefore based on a 1964-1965 national probability sample of 2,746 adults that is representative of the total population of persons aged 21 years or older living in households in the contiguous U.S.

Unfortunately, Cahalan et aj. (1969) analyzed their results on a quantity-frequency-variability measure and not on our definition of a problem drinker. They combined their quantity-frequency-variability measures to define a "heavy drinker." While one could be classified into the heavy drinker category in several ways, the minimum alcohol intake for a heavy drinker was five to six drinks at a setting two to three times a month. Using this definition, $12 \%$ of the sample were classified as heavy drinkers.

Men and women differ greatly in the proportion that are heavy drinkers. While on iy $5 \%$ of the women were found to be heavy drinkers, $21 \%$ of the men were classified as such. Age also was related to drinking practices. Cahalan et a1. (1969) found that approximately $15 \%$ of those from 21 to 49 were heavy drinkers, while $10 \%$ from 50 to 59 were heavy drinkers and only $6 \%$ of those 60 or older were heavy drinkers.

Family income was also related to drinking habits. Low income respondents were least likely to be heavy drinkers, while middle and upper income respondents were equally likely to be heavy drinkers. Education was also related to heavy drinking. Approximately $14 \%$ of those who had completed high school and $15 \%$ of those who had some college education were heavy drinkers, while those with more education or less education were less likely to be heavy drinkers.

Region of the country and community size were also related to drinking practices. Residents of the Middle Atlantic states were most likely to be heavy drinkers (19\%) followed by residents of New England (15\%) and the Pacific states (15\%). Residents from cities over one million population were most likely to be heavy drinkers (18\%) while residents from farms or small towns were least likely to be heavy drinkers (5\% 
to $8 \%$ ). Occupation is also related to drinking patterns. This relationship is discussed in much greater detail in the next section.

\subsection{Alcohol Abuse Prevalence Rates in the General Population}

The rates of alcoholism in the general population have generally been estimated in the past using the Jellinek (1960) formula. This formula predicts the number of alcoholics in a population based on estimates of the number of people who died from cirrhosis of the liver due to alcoholism. In 1948, Jellinek estimated that four million Americans were suffering from alcoholism using his definition. In 1965, the estimate was just over five million (Milt, 1974). While Jellinek's formula does permit for some variations in the estimates of alcoholism prevalence due to local variation in deaths from cirrhosis of the liver attributable to alcoholism, it essentially predicts that roughly $5 \%$ of a general adult population will be alcoholic.

However, there are some problems with the notion of estimating the prevalence of alcohol abuse based on Jellinek's approach. The formula does not allow for age, sex, and occupational differences in the population, all of which are known to be factors affecting the prevalence of alcohol abuse (Cahalan, 1970). Its lack of sensitivity to the effect of these factors and its underestimation of alcohol abuse by only basing its estimates on a single disease indicator, cirrhosis of the liver, have led to the more widely accepted use of the Marden formula.

The Marden formula was developed by Parker Marden (1980) based on nationa) survey data collected by Cahalan and his associates (Cahalan et al., 1969; Cahalan, 1970). The original Cahalan national survey on American drinking practices was carried out in 1964-1965 on a sample of 2,746 adults. Subsequently, a follow-up interview was conducted in 1967 on a subsample of 1,359 persons who had been interviewed on the original survey. In essence, Marden reanalyzed the Cahalan data, calculated a problem drinking score for each respondent based on 58 alcohol-related questions in the survey, chose a cut-off of seven affirmative answers or more out of the 58 items, and labeled those with a score of seven or more as problem drinkers. Thus, Marden's (1980) definition of problem drinking is the same as our definition of alcohol abuse and is directly relevant to this analysis.

The Marden formula has been recommended by the National Institute of Alcohol Abuse and Alcoholism (NIAAA) as the preferred method for est imating prevalence and the need for treatment in a given general population (State of Washington, Department of Social and Health Services, 1977). While the Marden formula is currently the best available method for estimating alcohol abuse prevalence, several issues should be kept in mind with regard to use of the formula. First, the data were collected on a random sample of aduit, U.S. household residents. Thus, the formula is meant to be used on somewhat comparable populations. It would not be a useful prediction formula, for example, on an indigent population. Direct application of the formula to nuclear industry personnel could also present such problems, since, without other 
data, there is no acceptable statistical method for estimating the errors of these estimates. It is difficult to tell whether the formula would underestimate, overestimate, or correctly estimate the alcohol abuse prevalence in this specific industry.

Second, the formula is based on a composite score from 58 items. There are no reliability indices reported for the score. In addition, there was no external criterion validity applied to the final score. That is, a person with a score of seven or more was, prima facie, considered to be an alcohol abuser and some external criterion, such as the spouse's report of alcohol-related problems, was not used as a validity check.

Third, the probabilities calculated for specific subgroups of the population were often based on a small sample size (N). Table 6 indicates that the probabilities for alcohol abuse based on sex and age were based on sample sizes that ranged from 140 people to 345 people. The sample sizes were much smaller for the probabilities for alcohol abuse among men based on age and occupation. While the exact N's for each cell in Table 7 are not available, data provided in Cahalan et al. (1969) suggest, for example, that there were approximately 68 men who were in the sales worker occupational category. Assuming an equal distribution of male sales workers across the five age categories, this means that the probabilities for a given age category within the sales worker occupational category are based on approximately 14 people. The smaller the sample size is, the larger the error of the probability estimate. Thus, for example, there would be more error associated with the est imates provided in Table 7 than for the estimates in Table 6 .

For the U.S. population as a whole, the Marden formula predicts that about 11\% of the aduit population from 20 through 69 has alcohol abuse problems. A finer breakdown of problem drinking, based on sex and age, is presented in Table 6 . The table indicates that the $11 \%$ problem drinking figure for the adult population is composed of an average of $17 \%$ problem drinking prevalence for men and $4 \%$ problem drinking prevalence for women. There are also obvious age differences. The highest prevalence for problem drinking of $13.4 \%$ is in the $20-29$ age group. However, this is due to the prevalence for men, since $24.8 \%$ of the men in this age group are problem drinkers. For women, the highest prevalence for problem drinking of $7.9 \%$ is in the 30-39 age group. In general, the prevalence of problem drinking drops off after age 49 .

The Marden formula prevalence estimates for men are also available as a function of age and occupation (see Table 7). Unfortunately, analogous data are not available for women since the original survey asked for the occupation of the male head of household and not for the occupation of the respondent. As shown in Table 7, the highest prevalence of alcohol abuse--approximately $22 \%$-- is found for four occupational categories operatives, transport workers, laborers, and farm laborers and foremen. The lowest prevalence of alcohol abuse--approximately $10 \%--$ is found for the professional/technical category. 
Table 6

Marden Formula Probabilities for Alcohol Abuse Based on Sex and Age

\begin{tabular}{|c|c|c|c|c|c|}
\hline \multirow{2}{*}{ Age } & \multicolumn{4}{|c|}{ Sex } & \multirow{2}{*}{ Average } \\
\hline & $\mathrm{Male}$ & $\mathbf{N}$ & Female & $N$ & \\
\hline $20-29$ & .248 & 216 & .020 & 256 & .134 \\
\hline $30-39$ & .164 & 242 & .079 & 345 & .122 \\
\hline $40-49$ & .174 & 264 & .068 & 333 & .121 \\
\hline $50-59$ & .127 & 197 & .019 & 265 & .073 \\
\hline $60-69$ & .116 & 140 & .012 & 195 & .064 \\
\hline $\begin{array}{l}\text { Average } \\
\text { (Total N) }\end{array}$ & .170 & $(1.059)$ & .044 & $(1,394)$ & .107 \\
\hline
\end{tabular}

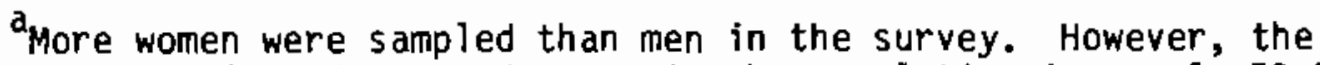
distribution of men and women in the population is nearly 50-50. Thus, the averages provided in the column assume an equal sex distribution. However, the average probability of alcohol abuse for men and for women is age-weighted. 
Table 7

Marden Formula Probabilities for Alcohol Abuse Among Men Based on Age and Occupation

\begin{tabular}{lcccccc}
\hline \multirow{2}{*}{ 0ccupation } & \multicolumn{5}{c}{ Age } & \\
\cline { 2 - 6 } & $20-29$ & $30-39$ & $40-49$ & $50-59$ & $60-69$ & \\
\hline Professional/technical & .111 & .151 & .113 & .089 & .042 & .101 \\
Manager/administrator & .250 & .250 & .122 & .127 & .171 & .184 \\
Sales workers & .261 & .136 & .097 & .078 & .053 & .125 \\
Clerical workers & .261 & .136 & .097 & .078 & .053 & .125 \\
Craftsmen & .250 & .146 & .280 & .177 & .179 & .206 \\
0peratives & .379 & .113 & .250 & .292 & .067 & .220 \\
Transport workers & .379 & .113 & .250 & .292 & .067 & .220 \\
Laborers (except farm) & .248 & .450 & .080 & .118 & .200 & .219 \\
Farmers \& farm managers & .261 & .136 & .097 & .078 & .053 & .125 \\
Farm laborers \& foremen & .379 & .113 & .250 & .292 & .067 & .220 \\
Service workers & .261 & .136 & .097 & .078 & .053 & .125 \\
Unemployed & .248 & .164 & .174 & .127 & .116 & .166 \\
\hline
\end{tabular}

assumes equal age distribution across occupational categories, since the age by occupation frequency counts are not available. 
In order to make the occupational data relevant to the nuclear utility industry, the major occupations in the industry were determined using the INPO (1982) survey of nuclear power plant occupations. The INPO survey did not analyze the number and $k$ ind of clerical and administrative staff. The relationship of the nuclear-related jobs to the occupational categories used in the Marden formula is presented in Table 8.

Our nuclear power plant job tit les were taken from the 1982 INPO survey of on-site nuclear-related employment at member utilities. Each job title was assigned to a Marden occupational category. Because the occupational categories specific to the nuclear industry were sometimes not listed in the census occupational category breakdown, the category assignment in Table 8 was based on the similarity of the nuclear-related occupation to listed occupation. Thus, some errors in occupational assignment in Table 8 are possible. Also, managers and administrators at nuclear plants were placed in the manager and administration category in Table 8 even though many of these personnel may have a professional or technical background. Where these personnel are placed would affect the alcohol abuse prevalence estimates for them.

\subsection{Nuclear Licensee Survey Findings on Alcohol Abuse*}

Respondents were hesitant to estimate the extent of alcohol-related problems in their plants. However, six of the eight respondents gave estimates of the alcohol abuse prevalence at their plants, based on personal observations. Five of the six indicated that $3 \%$ or less of the employee population had alcohol problems; one felt that as many as $9 \%$ of the plant's workforce were affected.

As noted in Section 4.4, in general neither the utilities nor the respondents kept detailed records of employee assistance program use. Therefore, respondents were unable to provide details of employee use of employee assistance programs or problems seen by employee assistance programs. However, seven of the eight respondents answered the question, "In your best judgment, has alcohol use increased or decreased since 1977?" Four of these said there had been "no change" in aicohol use during that period, two said alcohol use had increased, and one noted a decrease in alcohol use.

\subsection{Alcohol Abuse in Non-nuclear Industries}

All employee assistance program representatives surveyed reported that alcohol abuse is the single most comanon problem presented by employees seeking assistance from their programs. They concurred that at a minimum 10\% of those employed have alcohol abuse problems. An average of 50-60\% of all new cases handled by the employee assistance programs each year are related to alcohol abuse (see Table 9).

*The survey is discussed in more detail in Section 4.3 , above. 
Table 8

The Relotionshio of Wuclear Power Plant Job Categories to the

occoostional Categories Used In the Marden Formul

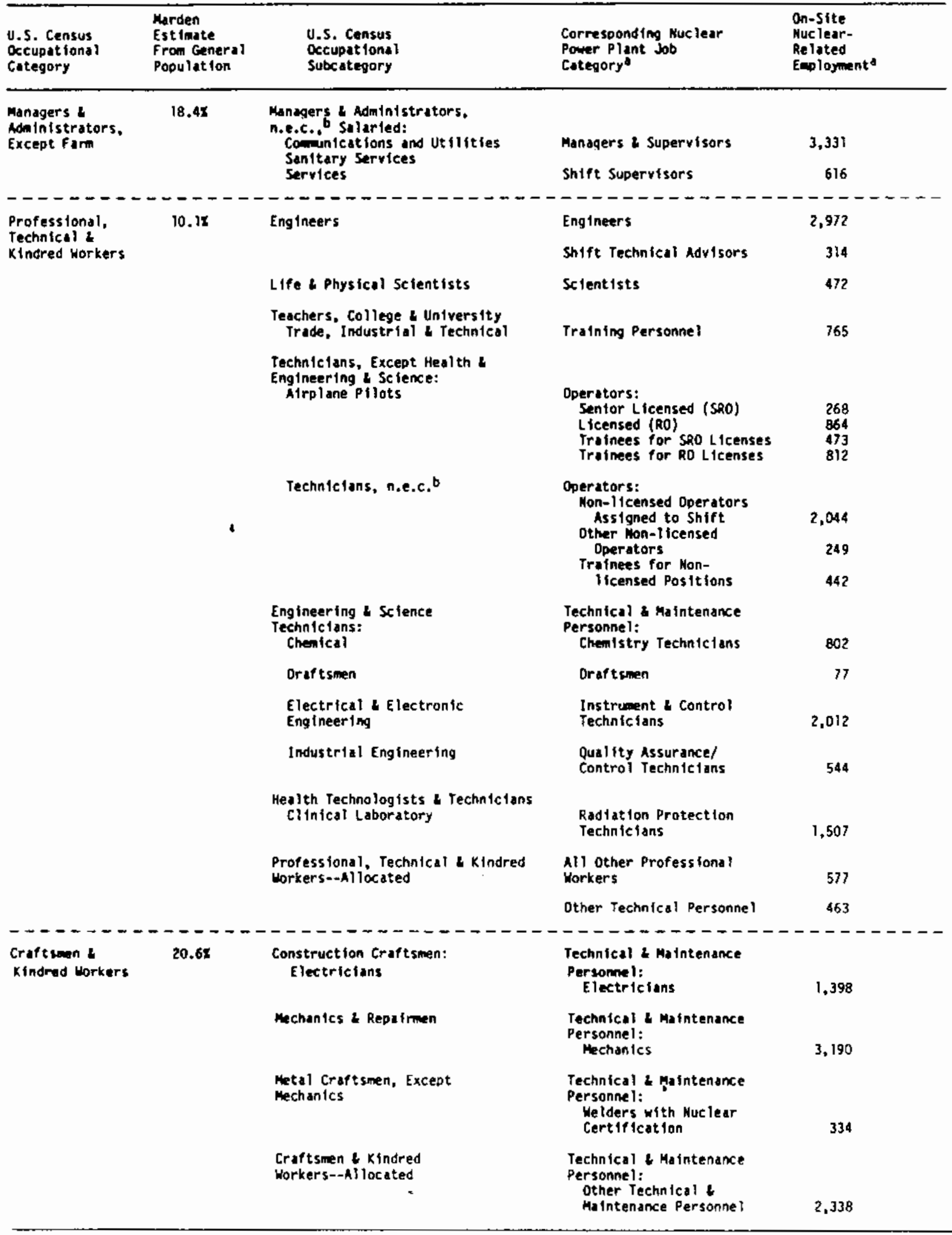

Taken from the INPO (1982) Occupational survey of the nuclear industry.

bun.e.c., means "not elsewhere classified." 
Table 9

Employees Reporting Drug-Alcohol Problems

over Past Five Years in Selected

Sample of Industries

\begin{tabular}{|c|c|c|c|c|c|}
\hline Year & $\begin{array}{l}\text { Total New Cases } \\
\text { out of } \\
\text { Total Emp loyed }\end{array}$ & $\%$ Drug & \% Alcohol & $\begin{array}{l}\text { \% Multiple } \\
\text { Substance }\end{array}$ & $\begin{array}{c}\text { Corporation } \\
A \text { to } G\end{array}$ \\
\hline 1977 & $\begin{array}{l}200 / 12,000 \\
120 / 4,000\end{array}$ & $\begin{array}{l}7 \% \\
5 \%\end{array}$ & $\begin{array}{l}70 \% \\
50 \%\end{array}$ & -- & $\begin{array}{l}A \\
B^{a}\end{array}$ \\
\hline 1978 & $\begin{array}{l}232 / 12,000 \\
120 / 4,000 \\
530 / 100,000 \\
30 / 1,500\end{array}$ & $\begin{array}{l}3 \% \\
5 \% \\
2 \%\end{array}$ & $\begin{array}{l}63 \% \\
50 \% \\
45 \%\end{array}$ & $\begin{array}{l}-- \\
54 \%\end{array}$ & $\begin{array}{l}A \\
B \\
C \\
D\end{array}$ \\
\hline 1979 & $\begin{array}{c}118 / 12,000 \\
120 / 4,000 \\
59 / 1,500 \\
116 / 13,000\end{array}$ & $\begin{array}{l}2 \% \\
5 \% \\
-- \\
--\end{array}$ & $\begin{array}{r}58 \% \\
50 \% \\
-- \\
100 \%\end{array}$ & $\begin{array}{l}-- \\
52 \% \\
--\end{array}$ & $\begin{array}{l}A \\
B \\
D \\
E\end{array}$ \\
\hline 1980 & $\begin{array}{c}173 / 12,000 \\
120 / 4,000 \\
74 / 1,500 \\
122 / 13,000\end{array}$ & $\begin{array}{l}2 \% \\
5 \% \\
--\end{array}$ & $\begin{array}{c}49 \% \\
50 \% \\
-- \\
100 \%\end{array}$ & $\begin{array}{l}-- \\
-- \\
62 \% \\
--\end{array}$ & $\begin{array}{l}A \\
B \\
D \\
E\end{array}$ \\
\hline 1981 & $\begin{array}{c}260 / 12,000 \\
120 / 4,000 \\
1,500 / 100,000 \\
85 / 1,500 \\
121 / 13,000 \\
720 / 8,500\end{array}$ & $\begin{array}{l}5 \dot{x}^{4 \%} \\
2 \% \\
-- \\
--\end{array}$ & $\begin{array}{c}47 \% \\
50 \% \\
45 \% \\
-- \\
100 \% \\
--\end{array}$ & $\begin{array}{l}-- \\
-- \\
29 \% \\
-- \\
--\end{array}$ & $\begin{array}{l}A \\
B \\
C \\
D \\
E \\
F\end{array}$ \\
\hline 1982 & $\begin{array}{l}378 / 12,000 \\
120 / 4,000 \\
300 / 22,200\end{array}$ & $\begin{array}{r}10 \% \\
5 \% \\
5 \%\end{array}$ & $\begin{array}{l}50 \% \\
50 \% \\
65 \%\end{array}$ & $\begin{array}{l}- \\
30 \%\end{array}$ & $\begin{array}{l}A \\
B \\
G\end{array}$ \\
\hline
\end{tabular}

-.. Not estimated.

astimates for Corporation B were made by the corporation's employee assistance program consultant and were constant from 1977 through 1982. 
The nature of the employee assistance programs' caseloads has changed over the last ten years. Representatives of employee ass istance programs that have been in existence for ten years or more reported that, formerly, middle-aged, male employees with severe alcohol problems were virtually the only type of referral received. Several factors may have influenced this early trend of referrals for alcoholism: first, only alcoholism treatment was offered; second, supervisor training focused on identifying symptoms of alcoholism with minimal relationship to job performance problems; and finally, only employees were eligible for services, with no assistance to the employee's family, who might be a part of the employee's problems. As could be expected under this structure, supervisors were reluctant to label an employee alcoholic and employees were reluctant to seek assistance, for fear of being labeled alcoholic. As a result, only those members of the workforce who reached advanced stages of alcoholism and remained visible on the job were referred for assistance, usually middle-aged male employees.

A more recent "broadbrush" approach to coping with job performance problems by employee ass istance programs changed the employee population seeking assistance. The broadbrush employee assistance program offers referrals for a variety of employee personal problems, including family and marital therapy, financial and vocational counseliling, and assistance with health or legal problems, in addition to drug and alcohol abuse evaluations and referrals. Under the broadbrush approach, the employee assistance programs focus on declining job performance. Supervisors are trained to refer people on the basis of inappropriate work behavior only, $r$ ather than attempting to diagnose the cause of the employee's poor performance. Responsibility for a limited diagnosis and referral rests with the employee assistance program counsellor once the employee contacts the employee ass istance program. Consequently, supervisors and employees are able to use the program without labeling the employee's problem as necessarily related to drug or alcohol abuse.

The shift to a broadbrush approach led to a larger number of referrals, a greater diversity in the type of employee seeking assistance (e.g., more women and younger employees, more management-level employees), more self-referrals, and earlier identification of problen-drinkers. Although alcohol abuse is still at the root of employee job performance problems in $50-70 \%$ of the cases referred to the employee assistance programs, the employees receiving assistance for alcoholism now represent all levels and types of employees within the organization. It was emphasized by all that alcohol abuse problems are not un ique to any one employee group; they occur with equal frequency among all employees, from top-level management down. These estimates of equal probability across occupational categories for alcohol abuse problems are not consistent with the estimates used by the Marden formula.

In addition to changes in program approach, changes have occurred in the nature of the employees' drug and alcohol abuse problems. The employee assistance program representatives reported that the majority (70\% or more) of their clients now present multiple alcohol/drug abuse problems, rather than just having an alcohol abuse problem. That is, the employee 
with drug or alcohol abuse problems is likely to be abusing one or more drugs in addition to alcohol. This finding is consistent with literature regarding the population in general (Carroll et al., 1977).

In summary, the interviews with employee assistance program personnel from fourteen organizations in the Seattle area indicate the following:

- A minimum of $10 \%$ of all employees experience alcoholrelated problems that affect their job performance.

- The most frequent problem of employees who seek assistance is alcohol-related.

- There is a greater tendency for drug and alcohol abuse problems to involve "multiple substance" abuse, alcohol in combination with one or more drugs; this trend is most commonplace among workers under 35 years of age.

- All categories of employees are equally affected by alcohol abuse.

- Employee assistance programs that have been set up to help personnel who are experiencing job performance decrements have been very successful and heavily used.

\subsection{Summary and Conclusions}

Alcohol abuse affects a large minority of adult U.S. citizens. Based on national survey data collected in the 1960 's, approximately $11 \%$ of the adult population between 20 and 70 are alcohol abusers. The prevalence of alcohol abuse is related to many factors, including sex, age, and occupation. Estimates based on these factors are available from the Marden formula.

The $11 \%$ alcohol abuse prevalence figure is composed of approximately 17\% of the adult male population and $4 \%$ of the adult female population. Alcohol abuse is more prevalent among the younger age categories, especially for men. For example, approximately $25 \%$ of the adult male population aged 20 to 29 were found to have alcohol abuse problems, while the analogous figure for 60 to 69 -year-old males is $12 \%$.

Alcohol abuse prevalence is also highly related to occupation. For example, national survey estimates for alcohol abuse among professional technical workers is approximately 10\%, whereas operatives, transport workers, and farm laborers have an estimated alcohol abuse prevalence of approximately $22 \%$.

An analys is of the job categories relevant to the nuclear industry found that nuclear-related jobs could be classified into one of three U.S. Census occupational categories. These three occupational categories are professional/technical, manager/administrator, and craftsmen. The Marden formula estimates based on general population data for these occupational 
categories are 10\% alcohol abuse prevalence among male professional/ technical workers in the general population, $18 \%$ alcohol abuse prevalence for male managers/ administrators, and $21 \%$ alcohol abuse prevalence for male craftsmen (analogous estimates for adult females are not available).

A general estimate used by employee assistance program consultants is that $5 \%$ to $6 \%$ of an average workforce needs employee assistance program help for alcohol abuse problems. If an all-male workforce was assumed, this estimate would range from $8 \%$ to $10 \%$. The estimates provided by Seattle-area employee assistance program representatives were that a minimum of $10 \%$ of their workforce needs help for alcohol abuse problems. Approximately $1 \%$ or less of their employee population seeks help through the assistance program during the year for alcohol abuse problems. Representatives from employee assistance programs at nuclear utilities generally estimated the alcohol abuse prevalence at their plant at $3 \%$ or less, although one representative estimated that the prevalence at his plant might be as high as $9 \%$.

As with the drug abuse prevalence estimates, a range of estimates for alcohol abuse prevalence in the general population and in non-nuclear industries is available. Without corroborating data, a specif ic prevalence estimate cannot be made, but the avajlable estimates are suitable for regulatory decisionmaking purposes. 


\section{IMPAIRING EFFECTS OF ALCOHOL ABUSE ON JOB PERFORMANCE}

In Section 5.1, a model of human performance was discussed that includes four types of performance: sensory/perceptual, cognitive, motor, and communicative. Five job categories in nuclear power plants (managers, scientists, licensed operators, non-licensed operators, and skilled craft workers) were also discussed in terms of the types of skills required for each job category and how these skills relate to the four human performance categories. This section briefly addresses the effects of alcohol on the four areas of human performance and, in turn, its potential impact on the five occupational categories. A more in-depth analysis of the impairing effects of alcohol on human performance is provided in Appendix $D$.

\subsection{Impairing Effects of Alcohol}

Alcohol has been found to impair performance on a wide variety of tasks, ranging from simple motor reflex responses to higher-level

problem-solving. Early inconsistencies in the experimental literature suggesting that large doses of alcohol improve performance have been set to rest. It is now accepted that alcohol produces performance decrements, some of which begin to appear at very low doses.

About $95 \%$ of the alcohol consumed is metabolized in the liver where it is changed to water and carbon dioxide before excretion. The rate at which alcohol is metabolized is slow and constant: about one-third ounce of pure ethanol per hour in the average adult. This metabolic rate is unaffected by the amount consumed, blood alcohol concentrations (BAC), or the consumption of food or other liquids (Wallgren \& Barry, 1970; AMA Comittee on Medicolegal Problems, 1970; Jones, 1978; Julien, 1981).

Like the sedatives, alcohol is a central nervous system depressant. At low doses, alcohol creates mild euphoria and behavioral excitement. This response has been interpreted by some as evidence that inhibitory synapses are depressed before excitatory ones, creating the state of disinhibition and arousal comonly noted after drinking begins (Julien, 1981; Perrine, 1974). With increasing doses, behavioral activity is progressively reduced to the point of coma or death.

Individual differences affect the onset of behavioral disruption due to alcohol consumption. Greater body weight, a slow speed of consumption, and physical tolerance to alcohol raise the BAC at which substantial behavioral disruption occurs. In general, however, disruption in human performance occurs at relatively low doses. In general, motor behaviors are impaired before cognitive abilities and recover later than cognitive skills, as the blood alcohol concentration rises, then falls, following alcohol ingestion. In the following discussion, BAC will be discussed in terms of the number of drinks required for the average American male. Table 10 summarizes the number of drinks equivalent to the blood alcohol concentrations given and the associated performance decrements. One drink is the equivalent of one ounce of hard liquor, $12 \mathrm{oz}$. of beer, or $3 \mathrm{oz}$. of unfortified wine. 
Table 10

Relationship of Levals of Blood Aiconol Concentrations and Types of Perforanice Decreenents

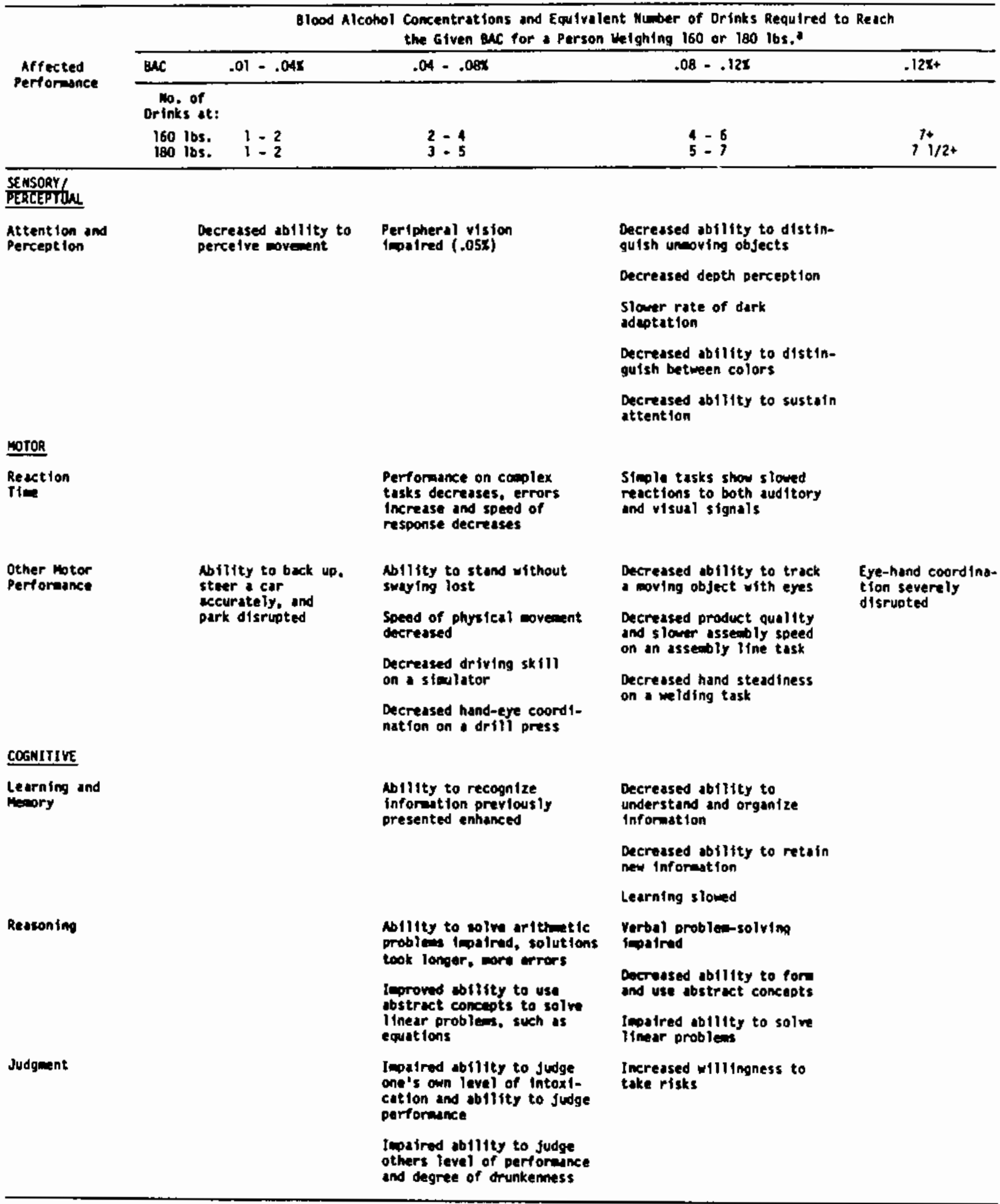

ulcohol is removed froe the bloodstren at the rate of $0.015 x$ per hour frrespactive of body weight. 
As can be seen from the table, alcohol effects show up at relatively low doses (one to two drinks). Performance decrements are found for a sensory/perceptual task (ability to perceive movement) and motor skills (aspects of driving skilis). At moderate doses (two to five drinks), sensory/perceptual, motor and cognitive performance decrements show up. Reduced ability to see and stand without swaying, longer reaction time, reduced eye-hand coordination, a reduced ability to solve arithmetic problems, and an impaired ability to judge one's own (and others) level of intoxication as well as performance ability are among those performance decrements observed.

Some performance factors are enhanced, however, at moderate dose levels. These performance capabilities are all cognitive and the effects are reversed with a few more drinks.

After four to five drinks, a number of human performance factors show deterioration. It is at four to five drinks that our average American male is verging on legal intoxication: most people whether practiced drinkers or not (i.e., alcohol tolerant) will show some behavioral effects of this dose level.

The combined effects of alcohol and other drugs on human performance have received limited scientific attention. Most research on the interactions focuses on the effects of drinking and tranquilizer (sedative) use due to the large numbers of tranquilizer prescriptions and the likelihood that individuals who are taking them will also drink. As marijuana use has become more common, some research has looked at its combined effects with alcohol. Given that multiple drug abuse is on the rise, it is likely that more information will be forthcoming on the effects of drug/alcohol interactions.

As noted in Section 5.4 , alcohol and sedatives are both central nervous system depressants. Consequently, they affect many of the same behaviors. When taken together, the resulting performance impairment is significantly greater than the impairment found when either alcohol or a sedative is taken alone.

The peak effects of an alcohol/sedative combination occur with in the first hour after both are ingested. Some evidence exists to suggest that alcohol accelerates the absorption rate of sedatives (Linnoila \& Mattila, 1973). Even if sedatives are taken up to ten hours before alcohol is consumed, effects of the combination are still observable (Linnoila, 1976).

Alcohol/sedative combinations significantly impair motor performance. Eye-hand coordination is decreased and reaction time greatly increased under the influence of low doses of these two drugs. In studies of simulated driving, subjects taking alcohol and a sedative were involved in more collisions, drove off the road more frequently, and ignored driving rules more often than persons taking either substance alone. 
Alcohol and sedatives are said to potentiate each other. As discussed in the sedative section (Section 5.5 ), potentiating interactions occur when the effects of dose combinations are more than simply additive. Therefore, with even small doses of both alcohol and sedatives taken in combination the behavioral effects include unsteadiness, disorientation, impaired judgment of intoxication, deficits in attention, and motor performance capabilities.

Different effects are found for alcohol/marijuana combinations. Marijuana, though not a central nervous system depressant, induces behavior effects similar to those found with alcohol (Julien, 1981). Unlike the alcohol/sedative combination, marijuana and alcohol do not combine synergistically; i.e., consumption of marijuana will not potentiate alcohol's action or vice versa. They do, however, impair performance to a greater extent than if either alcohol or marijuana is consumed alone. The combination particularly affects motor performance.

Cognitive performance is also affected by alcohol and marijuana taken together. In general, the combination of these two drugs significantly affects all aspects of human performance. Any ability which is negatively impacted by either drug alone will also be impaired by the combination.

\subsection{Effect on the Nuclear Industry}

As discussed above, alcohol has its earliest effects on motor performance, followed by sensory/perceptual performance, cognitive performance, and communicative performance. The impacts of these effects on the five nuclear job categories is summarized in Table 11 . In this table, only the high performance demand areas for a specific job category are evaluated.

Alcohol would appear to have the largest negative impact on the performance of non-licensed operators, licensed operators, and skilled craft workers. Alcohol abuse could definitely affect a licensed operator's performance. However, such performance problems should be relatively easy to isolate in the highly visible control room environment. In addition, errors in operation can be corrected by the remainder of the control room crew. Performance decrements due to alcohol abuse are of more concern if they cannot be easily detected. Such is more likely to be the case, for example, for maintenance personnel who work individually with little supervision or for non-licensed operators who perform, say, valve alignment checks individually. For these reasons, the negative effects of alcohol on job performance are likely to be the largest for skilled craft workers, non-licensed operators, and licensed operators, in that order. 
Table 11

Predicted Negative Impact of Alcohol on the High Frequency Performance Areas for Five Occupational Categories ${ }^{\mathrm{a}}$

\begin{tabular}{|c|c|c|c|c|c|}
\hline & Managers & $\begin{array}{l}\text { Scientists } \\
\text { E Engineers }\end{array}$ & $\begin{array}{l}\text { Licensed } \\
\text { Operators }\end{array}$ & $\begin{array}{c}\text { Non-Licensed } \\
\text { Operators }\end{array}$ & $\begin{array}{l}\text { Skilled } \\
\text { Craft } \\
\text { Workers }\end{array}$ \\
\hline $\begin{array}{l}\text { Sensory/ } \\
\text { Perceptual }\end{array}$ & & & Large & Large & \\
\hline Cognitive & Medium & Medium & Medium & & \\
\hline Motor & & & & Large & Large \\
\hline Communicative & Small & & Small & & \\
\hline
\end{tabular}

a The negative impacts of alcohol on performance were estimated only for the aspects of a specific job that had a "high" performance requirement as delineated in Table 4. For example, managers have high performance requirements for cognitive and communicative skills, so only those two cells were rated. 


\section{CONCLUSIONS AND RECOMMENDATIONS}

The conclusion to be drawn from the prior analyses is that some level of drug and alcohol abuse prevalence can be anticipated in the nuclear industry. With the lack of corroborating data, we must assume that the drug and alcohol abuse prevalence in the nuclear industry is likely to occur at approximately the same level as in the general population and in non-nuclear industries. Also, given the known effects of drugs and alcohol on sensory/perceptual, cognitive, motor, and communicative performance, we conclude that on-the-job drug and alcohol abuse will negatively impact job performance, as has shown to be the case in non-nuclear industries. Because of the above conclusions, we need to address the question of what $k$ ind of program can be implemented to minimize or eliminate on-the-job drug and alcohol abuse and the resulting performance decrements.

In the past, there have been two major types of programs that have been tried, especially with regard to alcoholism. The first approach tried (Wrich, 1980) was the alcoholic identification or supervisory identification approach. In this approach, supervisors are trained to identify the alcoholic on the bas is of alcoholism symptomatology. These programs were not successful for at least four main reasons. First, since first-line supervisors were reticent to diagnose anyone above them as being alcoholic, the identification of alcoholic workers was restricted to those below the first-line supervisory level. Second, the program of ten looked like a witch hunt. No matter how well the supervisors were trained, they tended to revert to decisionmaking on the basis of their own personal drinking habits. Third, alcoholics are very skillful at diverting attention from their real problem (alcoholism) to reasons why they drink. Supervisors were often fooled by the alcoholic and many troubled employees were not diagnosed correctly. Finally, because of the stigna that was attached to alcoholism in the 1950 's and early 1960's, supervisors wanted to be sure of their diagnos is before confronting the employee. Confidentiality was of ten compromised in the search for confirming data, and, again, troubled employees of ten went unhelped. Unfortunately, there are those who still advocate a symptom surveillance approach, especially for drug abuse (for example, see Bensinger, 1982).

In the 1960's, the programs began to shift their approach from alcoholism symptomatology to impaired job performance caused by alcoholism. At the same time, the program people realized that numerous personal problems-alcoholism, drug abuse, mental health problems, and family problems-could cause job performance decrements. Thus, the program became much broader in scope with the added benefit of finding that employees were much more likely to use a program that was in place to help them with their personal problems that caused job performance decrements than they were likely to use a program that was in place to treat only alcoholism. Such programs, which we have referred to as employee assistance programs in this report, have been very successful in the past decade. Program evaluations have shown that $70 \%$ to $90 \%$ of those who receive treatment 
through an employee ass istance program return to successfuliy carry out their job.

Much is already known about employee assistance programs. A comprehensive program should have the following elements (Segalla, 1982: p. 4):

- Written policy and procedures

- Labor-management cooperation

- Supervisory training

- Employee education

- Professional staff services

- Record keeping

- Ongoing evaluation

Although such general program features are known, specific programs will have to be tailored to specific needs. Variables that might affect program implementation include the number of employees, the availability of treatment services, and the kind of insurance coverage available.

Such programs have been successful because of the concern for employee prob?ems shown by the employer, by employee trust in the confidentiality assured by the program, and by the fact that job performance is the only criteria that is used in making referrals to the employee assistance program. Thus, it is our recommendation that employee assistance programs that include the program elements discussed above and that are based on decrements in performance, rather than on identifying the drug or a icohol abuser through symptomatology, be used in the nuclear utility industry. Such programs are acceptable to management, acceptable to labor, and successful. The programs that are based on identification through symptomatology have not been acceptable to labor and have not been successful. 


\section{REFERENCES}

American Medical Association Committee on Medicolegal Problems. 1970. Alcohol and the Impaired Driver: A Manual on the Medicolegal Aspects of Chemical Tests for Intoxication With Supplement on Breath/Alcohol Tests. National Safety Council, Chicago.

Bensinger, P.B. 1982. "Drugs in the Workplace." Harvard Business Review Nov-Dec:49-60.

Cahalan, D. 1970. Problem Drinkers. Jossey-Bass, San Francisco, California.

Cahalan, D. 1978. "Implications of American Drinking Practices and Attitudes for Prevention and Treatment of Alcoholism." In Behavioral Approaches to Alcoholism, eds. G. Marlatt and P. Nathan. Rutgers Center of ATcohol Studies, New Brunswick, New Jersey.

Cahalan, D. and R. Room. 1972. Problem Drinking Among American Men. Monograph No. 7. Rutgers Center of Alcohol Studies, New Brunswick.

Cahalan, D., et al. 1969. American Drinking Practices: A National Study of Drinking Behavior and Attitudes. Rutgers Center of ATcohol Studies, New Brunswick, New Jersey.

Campbel1, J.P., et al. 1970. Managerial Behavior, Performance, and Effectiveness. McGraw-HilT, New York.

Carrol1, J., et al. 1977. "The Meaning and Evolution of the Term 'Multiple Substance Abuse'." Contemporary Drug Problems Summer, 1977, pp. 101-133.

Chambers, C.D. 1971. Differential Drug Use With in the New York State Labor Force. New York State Narcotic Addiction Control Commission. Cited from a reference In Drug Abuse: Clinical and Basic Aspects, eds. S.N. Pradhan and S.N. Dutta. C.V. Mosby Co., St. Louis, 1977.

ChamberS, C.D. and J.A. Inciardi. 197la. An Assessment of Drug Use in the General Population. New York State Narcotic Addiction Control Comission. Cited from a reference In Drug Abuse: CTinical and Basic Aspects, eds. S.N. Pradhan and S.N. Dutta. C.V. Mosby, St. Louis, 1977.

Chambers, C.D. and J.A. Inciardi. 1971b. An Assessment of Drug Use in the General Population. Special Report No. 2. New York State Narcotic Addiction Control Commission. Cited from a reference In Drug Abuse: Clinical and Basic Aspects, eds. S.N. Pradhan and S.N. Dutta. C.V. Mosby Co., St. Louis, 1977.

Danielson, L.E. 1960. Characteristics of Engineers and Scientists. University of Michigan, Bureau of Industrial Relations, Ann Arbor, Michigan. 
Fishburne, P.M., H.I. Abelson, and I.H. Cisin. 1980. National Survey on Drug Abuse: Main Findings 1979. Superintendent of Documents, Washington, D.C.

Fitts, P.M. and M.I. Posner. 1967. Human Performance. Brooks/Cole Publishing Company, Belmont, California.

Frey, D. 1983. "What! Alcoholism in the Fire Service?" Speaking of Fire, Spring.

Gaston, M. and N.R. Walker. 1981. "Psychopharmacology." In Preparatory Course for the National and State Licensing Examinations in

psychology. Association for Advanced Training in the Behavioral Sciences, Berkeley, California.

Hodges, C.J., Jr. 1976. "Operator Tasks." In Conference Proceedings: Workshop on Power Plant Operator Selection Methods, ed. R.W. Pack. Report No. EPRI-SR-28. ETectric Power Research Inst itute.

Institute of Nuclear Power Operations. 1982. 1982 Survey of NuclearRelated 0ccupational Employment in United States Electric Utilities. AtTanta, Georgia.

Jellinek, E.M. 1960. The Disease Concept of Alcoholism. Hillhouse, New Brunswick, New Jersey.

Jones, E.R. and A.J. Eschenbrenner. 1982. "Simulators as Selection Devices for Nuclear Power Plant Operators." In The Human Equation in Electric Power Plants: A Symposium, ed. P.D. DuBois. Center for Nuc Tear Studies, Memphis State University, Memphis, Tennessee.

Jones, R.K. and K.B. Joscelyn. 1978. Alcohol and Highway Safety 1978: A Review of the State of Knowledge. DOT-HS-5-01277. U.S. Department of Transportation, National Highway Traffic Safety Administration, Washington, pp. 35-49.

Julien, R.M. 1981. A Primer of Drug Action. San Francisco: Freeman and Company.

Labor Department Employment and Training Administration. 1977. Dictionary of Occupational Titles. 4th Edition. U.S. Government printing office, Washington, D.C.

Levens, E. 1976. "The Cost-Benefit and Cost-Effectiveness of Occupational Alcoholism Programs." Professional Safety.

Linnoila, M. 1976. "Tranquilizers and Driving." Accident Analysis and Prevention 8(1):15-19.

Linnoila, M. and M.J. Mattila. 1973. "Drug Interaction on Psychomotor Skills Related to Driving: Diazepam and Alcohol." European Journal of Clinical Pharmacology 5:186-194. 
Marden, P.G. 1980. A Procedure for Estimating the Potential Clientele of Alcoholism Service Programs. U.S. Department of Heatth, Education, and Welfare PubTication No. (ADM) 80-908. National Institute on Alcohol Abuse and Alcoholism, Washington, D.C.

Milt, H. 1974. Basic Handbook on Alcoholism. Scientific Aids Publications, New Jersey.

National Institute of Alcohol Abuse and Alcoholism. 1978, 1981. 3rd Special Report to the United States Congress on Alcohol and HeaTth. 4th Special keport to the United states Congress on ATcohol and Health. United States Department of HeaTth and Human Services, Washington, D.C.

NUREG/CR-1750. 1981. "Analys is, Conclusions, and Recommendations Concerning Operator Licensing." National Technical Information Service, Springfield, Virginia.

NUREG-0903. 1982. "Survey of Industry and Government Programs to Combat Drug and Alcohol Abuse." National Technical Information Service, Springfield, Virginia.

Perrine, M.W. 1974. Alcohol Experiments on Driving-Related Behavior: a Review of the 1972-1973 Literature--Alcohol Countermeasures [iterature Review. DOT-HS-80T-266. U.S. Department of Transportation, Nationa] Highway Traffic Safety Administration, Washington, D.C.

Pradhan, S.N. and S.N. Dutta, eds. 1977 Drug Abuse: Clinical and Basic Aspects, C.V. Mosby Co., St. Lou is.

Scher, J.M. 1973. "The Impact of the Orug Abuser on the Work Organization." In Orug Abuse in Industry, ed. J.M. Scher. Char les C. Thomas, Springfield, ITlinois.

Segalla, E. 1982. "Employee Assistance Programs for Loca] Governments." Management Information Service Report 14(8): 1- 14 .

Sexias, F.A., et al. 1976. "A Definition of Alcoholism." Annals of Internal Medicine 5:764.

Smith, T.A. 1982. "A Structural Approach to Setting Up an Employee Assistance Program." Paper presented at the Nineteenth Annual Convention of the American Psychological Association, Washington, D.C.

State of Washington, Department of Social and Health Services. 1977. Washington State Alcoholism Plan, Fiscal Year 1978. Olympia, washington. 
Stephen, M. and R. Prentice. 1978. Developing an Occupational Drug Abuse Program: Considerations and Approaches. National Institute on Drug Abuse, Washington, D.C.

10 CFR 73.56, "Access Authorization Rule."

10 CFR 50.54, "F itness for Duty Rule."

Trice, H.M. and P.M. Roman. 1972. Spirits and Demons at Work: Alcohol and Other Drugs on the Job. CornetT University Press, Ithaca, New York.

Tuthi11, M. 1982. "Joining the War on Drug Abuse." Nation's Business June, pp. 64-65.

"TVA Issues Annual Report." 1980. The Reporter. The Newsletter of Federal Employee Health and Alcoholism/Drug Abuse Programs. VoT. 11, No. 8, August.

Urban, M.L. 1973. "Drugs in Industry." In Drug Use in America: Problem in Perspective, pp. 1136-1152. U.S. Government Printing office, washington, D.C.

U.S. Bureau of the Census. 1973. Census of Population: 1970 Subject Reports, Occupational Characteristics. Final Report PC(2)-7A. U.S. Government Printing office, Washington, D.C.

U.S. Nuclear Regulatory Commission. 1982. Information Notice 82-05. Washington, D.C.

Van Dyke, C. and R. Byck. 1982. "Cocaine." Scientific American 246: 128-134+.

Wallgren, H. and L. Barry. 1970. Actions of Alcohol. Elsevier, Amsterdam, Holland.

Wrich, J.T. 1980. The Employee Assistance Program. Hazeldon, City Center, Minnesota. 


\section{APPENDICES}




\author{
Appendix A \\ Interview Format for Utility Survey
}

Interview \#

ALCOHOL AND DRUG PROBLEM IDENTIFICATION SURVEY

TELEPHONE INTERVIEW PROTOCOL

I. I WOULD LIKE TO BEGIN WITH A FEW QUESTIONS ON EMPLOYEE ASSISTANCE PROGRAMS.

1. First, at your plant is there an employee assistance program that offers problem diagnosis, a referral service, or other human services?

No: Are you aware of whether there are adequate resources in the community to provide services if a referral system were to be set up? Yes

— No [NOW SKIP TO \#2]

Yes [CONTINUE WITH la, BELOW]

a. Is this program attached to a particular department or division?

$$
\text { No }{ }_{\text {Yes (please specify) }}
$$

b. What is the title of the person to whom you (or the head of the program) report(s)?

Titie

Department

c. How many people are assigned to work on the employee assistance program?

Full-t ime

Part-time 
d. When was the program established?

e. Does the employee assistance program ever get involved with staff performance evaluations?

No

Yes (please explain)

$f$. What services, if any, are specifically focused on alcohol- and drug-related problems?

__ problem diagnosis

_._ referral service

_ counselting

other (please specify):

g. Whom is this program designed to serve (all employees, or on ly some types of employees - please specify)? 
h. Next, could you give me some information on how many people have used the employee assistance program during the past several years.

[You may want to refer to program records to provide this information; if that requires lots of time or is inconvenient--could I make an appointment to call back?]

First, how many people total used the program in:

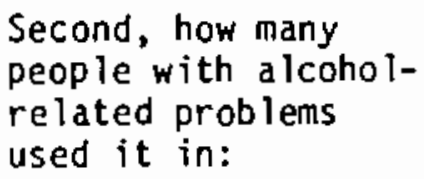

Second, how many people with alcoholrelated problems used it in:

Third, how many people with drugrelated problems used the program in:

1977

1978

1979

1980

1981

1982

j. Do you remember what $k$ inds of drug-related problems have surfaced this year - from January 1982 to the present? (E.g., marijuana, cocaine, tranquilizers such as valium, amphetamines, etc.)

Type of Drug

Percentage

2a. Do you know if there is an [another] employee assistance program in your utility?

No (SKIP TO \#3)

Yes, at the general of fices/headquarters

Yes, at other plant(s) 
2b. Do you know of a person I could contact about the program(s)?

HQ/Plant Name Individual's Name 
II. THE NEXT SEVERAL QUESTIONS ASK YOU TO MAKE JUDGMENTS ABOUT POSSIBLE ALCOHOL - AND DRUG-RELATED PROBLEMS AT YOUR PLANT--AND TO EXPLAIN HOW YOU ARRIVED AT THOSE JUDGMENTS.

3. What percentage of the staf $f^{\star}$ at your plant do you think have a problen related to the use of alcohol?

$$
\text { - } \%
$$

What type of information and impressions did you rely on in making this judgment? That is, how did you come up with this answer?

_ formal or informal written reports are filed word-of-mouth discussions your personal observations estimates based on personal opinion estimates compiled by the employee assistance program records kept by the employee assistance program records kept by Personnel Department other (please specify):

4. What percentage of the plant staff do you think have a problem related to the use of a drug?

\section{\%}

What type of information and impressions did you rely on in making this judgment? That is, how did you come up with this answer? formal or informal written reports are filed word-of-mouth discussions your personal observations estimates based on personal opinion estimates compiled by the employee assistance program records kept by the employee assistance program records kept by Personnel Department other (please specify):

*Staff $=$ all personnel with unescorted access to protected areas. 
5. Do some groups of workers have more of a problem than other groups?

No
Y. Yes (please explain--what groups; type of problen--
alcohol vs. drug):

What type of information and impressions did you rely on in making this judgment? That is, how did you come up with this answer?

_ formal or informal written reports are filed word-of-mouth discussions your personal observations estimates based on personal opinion estimates compiled by the employee assistance program records kept by the employee assistance program records kept by Personnel Department other ( $p$ lease specify): 
6. In your best judgment, has alcohol use increased or decreased since $1977 ?$
_ increased
decreased no change

What type of information and impressions did you rely on in making this judgment? That is, how did you come up with this answer?

formal or informal written reports are filed word-of-mouth discussions your personal observations estimates based on personal opinion estimates compiled by the employee assistance program records kept by the employee assistance program records kept by Personnel Department other (please specify):

7. In your best judgment, has drug use increased or decreased since 1977?
increased decreased no change

What type of information and impressions did you rely on in making this judgment? That is, how did you come up with this answer?

formal or informal written reports are filed word-of-mouth discussions your personal observations estimates based on personal opinion estimates compiled by the employee assistance program records kept by the employee assistance program records kept by Personnel Department other ( $p$ lease specify): 
8. If you like, when our research report is completed--somet ime next year, we will send you information on how to obtain a copy of the report (if it is available from the GPO) or a copy of the report itself (if it is not published by the GPO). Do you want this information?

No
Yes: May I get your:

Name:

Title:

Plant:

Address:

Should you have any further comments or questions, please don't hesitate to call me at:

(206) $525-3130$ OR WRITE AT:

Battelle Memorial Institute

4000 N.E. 4 lst Street

Seattle, Washington 98105

THANK YOU VERY MUCH FOR YOUR TIME AND ASSISTANCE. 


\section{Final Questions--the Interview Protocol}

1. As you think back on this interview, was there any point at which you found yourself feeling irritated with me or with the questions?

__ No

2. At any time during the interview did you feel uncomfortable or uneasy?

${ }_{-}$No 


$$
\begin{gathered}
\text { Appendix B } \\
\text { Local Industries Surveyed } \\
\text { October, } 1982 \text { - January, } 1983
\end{gathered}
$$

\section{Industry}

Burlington Northern Railroad

The Boeing Company

Brittania Sportswear, subsidiary of Schoenfeld, Inc.

City of Seattle

Highline School District

King County Division of Alcohol and Substance Abuse

Pacific Car and Foundry Company

Pacific Northwest Bell

Puget Sound Naval Shipyard

Seattle First National Bank

The Seattle Times Company

Washington Natural Gas Company

Western Airlines International

Heyerhauser Company
Date

November 1982

December 1982

December 1982

December 1982

December 1982

December 1982

December 1982

December 1982

January 1983

October 1982

Decenber 1982

December 1982

Oecember 1982

December 1982 
Appendix C

Impairing Effects of Drugs on Human Performance

MARI JUANA

As the most commonly used drug, other than alcohol, the effects of marijuana on human performance have received increasing attention in the scientific literature over the past ten years. Delta-g-

tetrahydrocannibol (THC) is the active ingredient in marijuana and in hashish, a marijuana-derivative made from resins in the female flowers of the Indian hemp plant, Cannabis sativa. Ingestion or inhalation of THC increases pulse rates and dilates the blood vessels in the eyes. Absorption from the lungs is rapid and complete, and peak blood concentrations occur 10 to 30 minutes after smoking a marijuana cigarette. Effects of the drug last typically for 2 to 3 hours (though some effects have been noted for up to $41 / 2$ hours), but excretion of marijuana metabolites may require a week or longer. Marijuana use is not physiologically addicting, despite its legal classification as an addicting drug under the Controlled Substances Act. Some users have reported a psychological dependence on this drug, however, and tolerance to its psychological and physjological effects develops with repeated use.

Most marijuana sold in the United States contains THC concentrations ranging from $0-5 \%$, while THC concentrations in hashish typically range from 5-20\%. In the low doses commonly ingested, marijuana produces behavioral effects similar to the sedatives; only with extreme Iy high doses will marijuana produce hallucinations and other psychedelic effects. The studies to be discussed in this section have used a typical "social dose," or one or two marijuana cigarettes shared by two or three people. Subjects administered this typical "social dose" are referred to as being intoxicated in the following review.

The effects of marijuana intoxication on an individual's sensory/perceptual performance have been investigated by a number of researchers. Studies have been conducted to examine the effects of marijuana on sight and hearing and on the ability to sustain attention while intoxicated.

Two studies have investigated the effects of marijuana on hearing. Early research showed that marijuana did not impair the ability to detect sounds near the lower limits of human hearing or to discriminate between tones of different frequencies and amplitudes (Caldwell, et al., 1969). In Caldwe]1, et al.'s study, however, subjects controlled their own consumption of marijuana, so may not have ingested enough of the drug to produce an effect. A more recent study, which controlled the amount of marijuana ingested, found significant impairment related to the amount of marijuana (Moskowitz \& McGlothlin, 1974). In this study, subjects were asked to report the presence or absence of a tone presented to one ear in a burst of noise while either ignoring or attending to a human voice reading a list of numbers to them in the other ear. Marijuana intoxication decreased the number of times the subjects reported that the tone was present when it actually was present (hits), and increased the 
number of times they reported that it was present when it was not (false alarms). The results indicated that the impairment was due to a decrease in sensitivity to the auditory signals, rather than to changes in the criteria the subjects used as a basis for reporting the presence of a signa 1.

Several studies have investigated the effects of marijuana on vision and found no effect. Perception of the brightness of a light stimulus was unaffected (Caldwell, et al., 1969) by marijuana, as was performance on depth perception tasks and the ability to discriminate rapidly changing lights as discrete light sources (Clark \& Nakashima, 1968; Peters, et al., 1976). Dark adaptation, visual acuity, and visual recognition were similarly unaffected (Milstein, et al., 1975; Moskowitz, et al., 1972).

Performance decrements on other vision tasks have been found, however, in a number of studies. Sharma and Moskowitz (1972) found that intoxicated subjects reported more movement of light source. Other researchers have found that marijuana is associated with decreased sensitivity to central and peripheral visual signals (LeDain, et al., 1972; Moskowitz, et al., 1972; Macavoy \& Marks, 1975). In a study of visual information processing, however, Braff, et al. (1981) concluded that marijuana does not affect visual intake capacity, image formation or quality, which suggests that visual sensitivity is not impaired with marijuana intoxication. Rather, these researchers found that marijuana slows the transfer of a visual image from iconic (image or visual) memory to more permanent registration and processing. Iconic memory may become over loaded and the images may become fused or lost before transfer can occur. That is, the sensory input is unimpaired but the slowing of the storage process reduces the accurate recording of the visual input.

Another aspect of sensory/perceptual performance, the ability to attend to stimuli, is also affected by marijuana intoxication. Several studies have demonstrated that the ability to sustain attention is impaired with marijuana (Moskowitz, et a)., 1972; Casswell \& Marks, 1973). Sharma and Moskowitz (1973) found that vigilance performance on a simple signal detection task was impaired; performance further decreased over repeated exposure. A similar finding was obtained on a more complex and demanding vigilance task (Sharma \& Moskowitz, 1974), suggesting that performance decrements were not solely due to lapses in subject interest in the first experiment.

Performance decrements have not been found, however, on tasks which require continuous performance (Weil, et ai., 1968; Vachon, et al., 1974; Casswe 11, 1975). Casswell (1975) suggests that the relatively long response time allowed on the continuous performance task obscures brief lapses of attention due to marijuana intoxication, whereas the vigilance tasks allow less response time and so are more sensitive to attention decrements.

Marijuana intoxication does create substantial performance decrements on tests of motor skills; many of these decrements are long lasting, showing significant deficits for as long as four and one-half hours after the administered "social" dose. Reaction times are longer. A number of 
studies have found impairments in performance on both simple and complex reaction time tasks (Peters, et al., 1976; Clark \& Nakashima, 1968; Schaefer, et al., 1977; Chesher, et al., 1977), whether the stimulus was presented visualiy or auditorially (Bird, et al., 1980). Further, the impairment was found to be relatively long-lasting: up to four and one-half hours following drug ingestion (Belgrave, et al., 1979b). Widely ranging differences in subjects' performances on reaction times led one researcher to suggest that some of the impairment may be due to brief lapses of attention (Casswel1, 1975), although a slowing of muscular movements with intoxication has also been noted (Borg, et al., 1975). Early increases in reaction time for complex tasks do disappear with practice on the task, however. In one study, practiced subjects showed no marijuana-induced impairments (Peeke, et a 1., 1976).

Studies of several kinds of motor performance indicate that marijuana impairs motor steadiness, eye-hand coordination, and manual dexterity. In contrast to the reaction time tasks, which require some information processing, performance on tests of simple motor reaction speed (e.g., finger and toe tapping) was unaffected by marijuana intoxication (Milstein, et al., 1975; Peters, et al., 1976).

Motor steadiness is severely impaired with marijuana. Standing steadiness with eyes both open and closed was decreased after ingestion of marijuana (Belgrave, et al., 1979; Chesher, et al., 1977; Bird, et al., 1980), as was hand steadiness (Milstein, et al., 1975).

Performance decrements on sustained tracking tasks have consistently been found. Subjects show slower response times and increased time off-target when intoxicated (Milstein, et a1., 1975; Belgrave, et al., 1979b; Roth, et al., 1973; Bird, et al., 1980).

Manual dexterity performance is also impaired with marijuana intoxication. Performance on tests requiring manipulation and coordination skills has shown significant impairment due to marijuana ingestion (Salvendy \& McCabe, 1975). Another study which tested for manual dexterity found performance decrements which lasted for up to approximately two and one-half hours after ingestion of marijuana (Chesher, et al., 1977).

The scientific literature on the effects of marijuana on cognitive performance yields inconsistent results. For example, the effects of marijuana on memory and learning have been found to produce both significant performance decrements in some instances and none in others. The differences may be a function of non-comparable experiments or tasks in the cognitive arena which is, admittedly, a complex category of human performance. On the other hand, the differences may be due to inherent properties of marijuana which, to date, have been found to be unlike those of any known neurotransmitter in the brain. The studies reviewed include investigations of memory, learning, reasoning, and decisionmaking on tasks requiring judgment. 
Differences have been found for memory retrieval tasks when the subject is intoxicated at the time of learning material to be retrieved later. A number of studies indicate that marijuana does not interfere with retrieval of material previously learned in a non-intoxicated state (Abe1, 1971; Darley \& Tinklenberg, 1974; Dittrich, et al., 1973; Dornbush, 1974). For example, Darley, et a1. (1977) found that marijuana had no effect on the ability to recali or recognize common facts stored in long-term memory. Miller, et al. (1977a), however, found a slight retrieval deficit using a more sensitive experimental design, but this deficit was overcome when the subjects were provided with cues to aid the ir recall.

Several studies have indicated that marijuana interferes with the transfer of information from short-term memory to long-term memory. Immediate recall of simple information presented while the subjects were intoxicated was not impaired by marijuana (Peters, et a1., 1976; Dittrich, et al., 1973), but Miller, et a1. (1977b) found that inmediate recall of complex prose material decreased with marijuana use. Recall of material from long-term memory, learned while intoxicated, was significantly impaired in a number of studies (Miller, et al., 1977a; Rickles, et al., 1973; Pearl, et al., 1973; Miller, et al., 1972).

Marijuana has been found to interfere with learning. In a study of verbal learning, Rickles, et a1. (1973) found that intoxicated subjects required more trials to reach a criterion than non-intoxicated subjects. Peters, et al. (1976) also found that intoxicated subjects required more practice to learn a color-number matching task than subjects receiving a placebo.

One consistent finding has been that marijuana impairs performance on the digit symbol substitution task. This task gives subjects a code which pairs numbers and letters. When presented with a letter, the subject is required to respond with the appropriate number, according to the code. As the code is learned, subject response time and number of errors should decrease. Several studies found significant performance decrements on this task for intoxicated subjects, but the impaired performance may be due to slower reaction time or reduced speed of information processing, rather than to a failure to learn (Clark \& Nakashima, 1968; Casswel1, 1975; Vachon, et al., 1974; Borg, et al., 1975).

Some evidence of state-dependent learning was found in two studies. State-dependent learning refers to learning that is best recalled when the subject is in the same state (e.g., intoxicated) as when the learning occurred. Information learned while intoxicated was recalled better after a delay by subjects who were again given marijuana than by subjects who were asked to recall the material in a non-drug state (Miller, et al., 1977a; Rickles, et al., 1973). In both studies, however, material learned while intoxicated was recalled to a lesser degree, independently of drug state, than material learned without the inf luence of marjjuana.

The effects of marijuana intoxication on reasoning abilities indicate that numerical reasoning is significantly impaired, whereas the formation 
and use of abstract concepts is impaired for some tasks, but not others. As was noted in the reaction time research, the response time for reasoning tasks substantially increases with marijuana.

Several studies have found impaired numerical reasoning with marijuana. Chesher, et al. (1977) found performance decrements on simple addition and subtraction tasks for intoxicated subjects, as did Belgrave, et al. (1979b) on a timed test. Intoxicated subjects showed longer response times and made more errors than non-intoxicated subjects in these studies. Pear 1 , et al. (1973) found a significant, dose-related effect of marijuana on a goal-directed serial alternation (of addition and subtraction) task, and on a serial subtraction task. In this study, however, motivated subjects made fewer errors when intoxicated than subjects not given an incentive to perform well.

Marijuana has been shown not to impair performance on tests which require the subject to choose the element in a set which is not conceptually related to the other elements. No performance decrements were found on tests which presented words as the set (Pearl, et al., 1973) or geometric figures (Peters, et al., 1976) as the set.

Pearl, et al. (1973) found that marjjuana reduced performance on two other reasoning tests, however. Intoxicated subjects made more errors when presented with an ordered series of letters and required to deduce which letter should come next in the sequence. Intoxicated subjects also made more errors when presented with a series of pictures with parts missing and asked to identify the subject of each picture.

Very little information is available regarding the effects of marijuana on judgment. No studies of decisionmaking while intoxicated have been conducted, although the reaction time, memory, and reasoning data suggest that judgment may be impajred.

Studies of interpersonal behavior have indicated that individuals become less empathic when intoxicated. One study of mental health workers found that they were less able to accurately perceive emotion in others or to communicate genuinely when intoxicated (Janowsky, et al., 1979). Similarly, clopton, et al. (1979) found that subjects were significantly less able to identify the emotions of actors in video-taped interactions when intoxicated than when in a non-drug state. Social judgment, therefore, appears to be impaired with marijuana.

The effects of marijuana on communication performance have not been studied. Communication, however, can be expected to be influenced to the extent that complex reaction time, memory, numerical reasoning, and social judgment are involved in the creation of specific verbal. behavior. As indicated in the preceding discussion, all of these skills are affected by marijuana ingestion.

Chronic (i.e., long-term and frequent) abuse of marijuana does not appear to lead to the physical and psychological deterioration associated with chronic abuse of other drugs (e.g., stimulants, sedatives, and alcohol). 
Even large daily doses of THC do not create physical dependence (Marcovitz \& Myers, 1944; Deneau \& Kaynakcalan, 1971; Harris, et al., 1972), though psychological dependence has been reported (Bouquet, 1944; Chopra \& Chopra, 1957; Lambo, 1965).

Experimental studies of the effects of chronic marijuana abuse on performance are rare. Two studies examining the effects of daily consumption on work productivity over periods of 70 and 93 days, respectively, showed no effect on productivity as long as subjects were not required to work while intoxicated (Miles, et al., 1975; Kagel, et al., 1980). The amount of marijuana ingested did not affect the results. The time period involved in these studies, ten weeks to approximately three months, may not be representative of effects over years of heavy use.

Studies conducted in the Carribean, the Middle and Near East, and Central Europe of long-term heavy cannabis users fail to provide evidence of significant and permanent impairment due to chronic ingestion of THC. Results of several studies suggest that chronic users of THC are indistinguishable from the ir peers in terms of work performance, mental status, social functioning, and measures of lifestyle ( $F$ ink, 1971; Rubin \& Comitas, 1972; Weiss, 1971; Mendhiratta, et al., 1978).

As a result, it can be concluded that chronic use of marijuana is not linked to permanent or sustained performance decrements; the impact of marijuana use on job performance must be attributed to marijuana consumed on the job or several hours prior to work shifts. In spite of evidence of marijuana metabolites being present in the body for days after ingestion, no link with performance decrements has been established. The apparent effects of marijuana abuse are relatively short lived, though certainly significant in terms of performance capabilities.

Studies of the effects of marijuana on performance when combined with other drugs have been limited to the combination of marijuana and alcohol. These studies have consistently shown that the effects of these two drugs are additive, so that any impairment found for either drug will also be found when both drugs are taken together (Macavoy \& Marks, 1975; Belgrave, et al., 1979b; Chesher, et al., 1977; Bech, et al., 1973; Bird, et a 1., 1980). Marijuana and alcohol do not combine synergisticaliy; i.e., consumption of marijuana will not potentiate alcohol's action or vice versa.

In sumenary, though the findings of the scientific literature are not entirely consistent, marijuana influences an individual's ability to perform a number of sensory/perceptual skilis. Decreased hearing ability as well as a decreased ability to ejther recognize or process visual stimuli is reported. Also, evidence points to a reduced ability to sustain attention in a vigilance task.

Marijuana intoxication is associated with significant motor performance decrements on a variety of reaction time, motor steadiness, manual dexterity, and eye-hand coordination tasks. In some instances these 
decrements continue for as long as four and one-half hours after ingestion of the marijuana. Practice of the motor skills involved in reaction time for complex tasks may reduce performance decrements.

The effects of marijuana on cognitive tasks are not readily understood. In general, however, marijuand intoxication affects memory when the material to be recalled is learned in an intoxicated state. Learning is also affected by marijuana intoxication. Evidence for state-dependent learning has been found. Numerical reasoning is significantly impaired; other forms of reasoning were not consistentiy affected by marijuana intoxication. No data are available on marijuana intoxication and decisionmaking. Several studjes of interpersonal judgment suggest that social behavior is impaired by marijuana intoxication.

STIMULANTS

The class of stimulant drugs includes the amphetamine famjly and cocaine. The amphetamines and cocaine are functionally similar. The major functional difference is one of intensity and duration of effect.

The amphetamines are synthetic products. They are commonly avajlable in three forms--amphetamine, dextroamphetamine, and methamphetamine. The effects of these three forms are qualitatively similar. However, for a given dose of the drug, amphetamine will produce the least intense effects, and methamphetamine will give the most intense effects. A standard low dose of amphetamine is by tablet form from 5 to $50 \mathrm{mg}$.; high doses are above $100 \mathrm{mg}$. A low dose of dextroamphetamine is 2.5 to $20 \mathrm{mg}$; high doses are above $50 \mathrm{mg}$. Low doses of methamphetamine are less than 2.5 to $20 \mathrm{mg}$. , and high doses are less than $50 \mathrm{mg}$.

The amphetamines have few legitimate medical uses. They are prescribed (rarely) for treatment of narcolepsy and petit mal epilepsy. Amphetamines are also prescribed for appetite suppression. However, the effect for a constant daily dose lasts only about two weeks, and appetite returns to norma] unless the amphetamine dose is increased. Cocaine is used medically for local anesthesia and shrinkage of mucous membranes in surgery, although newer forms of anesthesia are typically substituted.

The potential for abuse of the amphetamines and cocaine is great because of their psychological effects. Their dominant effects on the subjective state are increased alertness (particularly if the user is fatigued) and feelings of well-being. Effects depend on form of drug used, dosage, and method of administration. Some effects diminish with continued use of the same dose (for example, appetite suppression), whereas others do not (e.g., the awakening effects). For many stimulant abusers, tolerance to some effects leads to a pattern of escalating dosage to achieve the desired effects. This escalation results in several-day "runs" of no sleep, followed by "crashes" when the user stops taking amphetamines. The characteristics of crashes include severe emotional depression, fatigue, profound and prolonged sleep, and increased appetite. 
The effect of stimulants on human performance has been evaluated in the scientific literature; however, none of the experimental work reviewed was done with cocaine. Though there are few differences between cocaine and amphetamines, the conclusions drawn regarding performance effects of stimulants may differ from performance effects resulting from cocaine abuse.

Vigilance performance, or the ability to attend to sensory input, has been improved in fatigued subjects given low doses of amphetamines (Paine \& Hauty, 1954, 1955; Hauty \& Paine, 1958). When administered to subjects who were not in a fatigued condition, no differences were observed in visual acuity or the ability to recognize a given stimulus in a reaction time task (Frowein, 1981).

Motor performance is also affected by stimulants. Athletic performance (swimming, running, etc.) is improved significantly in nonfatigued subjects given doses of amphetamines (Smith \& Beecher, 1959). The learning or acquisition of athletic motor skills has also been noted to improve (Spiege1, 1979). Tests of reaction time show fatigued and nonfatigued subjects react more rapidly on a variety of simple and complex tasks (Laties \& Weiss, 1966; Kornetsky, et a1., 1959; Seashore \& Ivy, 1953; Frowein \& Sanders, 1978; Frowein, 1981). Improved coordination in subjects also has been noted (Weiss \& Laties, 1962). The only adverse motor response seems to be evidence of fine tremors in unf at igued subjects (Spiegel, 1979).

The effect of stimulants on cognitive performance is less straightforward. In general, simple cognitive performance has been noted to improve in subjects given stimulants; performance on simple repetitive tasks which typically elicit fatigue and boredom is improved (Smith, et al., 1963; Weitzner, 1965), fewer errors are found on tests of simple math (Forrest, et al., 1967), and higher scores are recorded on speeded tests of verbal ability (Vaness \& Brown, 1966; McDonald, 1972). Learning also appears to be improved with stimulants ( $L a l$, et al., 1972); evidence exists for greater retention of visual information (Vojtechovsky \& Safratova, 1971; Hurst, et a1., 1968) and an enhanced ability to learn to read and understand a foreign language, specifically Chinese (Kupietz, et al., 1980).

Limited evidence exists to indicate that more complex cognitive performances are unaffected by stimulants. No enhancenent of short-term memory skills has been found (Mohs, et al., 1978) and amphetamines have been found to have no effect on performance tests requiring higher leve] cognitive functioning such as is used in calculus (Barmack, 1940). Most of the positive effects of amphetamines on cognitive performance are generally attributed to the drug's ability to enhance attention and alleviate boredom on simpler repetitive tasks rather than enhance thinking and judgment in complex cognitive tasks (Frowein, 1981).

With respect to communicative performance, stimulants increase the amount of talking in a social situation (Griffiths, et al., 1977). 
To summarize, stimulants generally enhance human performance. In nonfatigued subjects several motor, cognitive, and communicative skills are enhanced. In fatigued subjects, attentional and motor capabilities are returned to at least normal range. The generally positive performance effects of stimulants undoubtedly account for the ir popularity among college students, long-distance truck drivers, and athletes.

The effects of chronic use of stimulants are several. Tolerance develops quickly (approximately two to four weeks if used daily) and therefore increasing doses must be administered to achieve the same behavioral effects. Tolerance is thought not to occur, however, with cocaine ( $V$ an Dyke \& Byck, 1982). Sleep disturbances occur with stimulant abuse; prolonged abuse also leads to physical damage, massive depression when the effects wear off (suggestive of physiological withdrawal), compulsive repetitive behavior and paranoid psychos is that is indistinguishable from naturally occurring psychotic disorders.

\section{SEDATIVES}

The sedatives are the most commonly physician-prescribed drugs, and although they differ somewhat in chemical composition and site of action in the body, all drugs in this class are central nervous system depressants. Familiar sedatives are the barbiturates, the anxiolytics or minor tranquilizers, ethanol, and the general anaesthetics. At low doses, all of these drugs are capable of producing behavioral disinhibition and euphoria. At higher doses, each of them produces drowsiness, and can induce unconsciousness if taken in sufficient amounts (Julien, 1981).

The drugs in this class which are most likely to be used by employed individuals are the minor tranquilizers, the barbiturates, and alcohol. The effects of alcohol on human performance are discussed in Section 7. The action and effects of the minor tranquilizers and the barbiturates are described below.

Anxiolytics

Included in the minor tranquilizers are the benzodiazepines and the dicarbamate derivatives. There are over 2,000 benzodiazepine compounds; the ones most commonly prescribed are diazepan ("Valium"), chlordiazepoxide ("Librium"), and flurazepam ("Dalmane"). Among the dicarbamates, meprobamate ("Miltown" or "Equanil") is the most widely prescribed. For the purposes of the following discussion, all of these will be referred to as minor tranquilizers or by commonly known brand names. These drugs are effective in the treatment of tension, anxiety, and psychosomatic disorders. They are also used for treatment of alcoholism and phobic states (Gaston \& Walker, 1981). Valium is prescribed for symptomatic relief of anxiety and tension, alleviation of the symptoms of acute alcohol withdrawal, relief of muscle spasms, treatment of convulsive disorders, alleviation of pre-surgical anxiety, and as a hypnotic (sleeping pill). Standard doses for these purposes 
range from 2 to $10 \mathrm{mg} .$, taken orally 2 to 4 times daily. In some cases the drug is administered by injection intramuscularly or intravenously in doses ranging from 2 to $15 \mathrm{mg}$. (Physician's Desk Reference, 1982). By this route of administration, $V$ alium has a more rapid effect and reaches blood levels approximately three $t$ imes faster than those obtained by oral ingestion (Hillestad, et al., 1974; Orr, et al., 1976). Dalmane is prescribed specifically as a sleeping pill (i.e., hypnotic). Standard doses are 15 or $30 \mathrm{mg}$., taken orally at night before retiring. Miltown is prescribed for relief of anxiety and tension and for promotion of sleep in anxious, tense patients. The usual dosage is 1200 to $1600 \mathrm{mg}$. per day, administered orally in 3 or 4 doses (Physician's Desk Reference, 1982).

The effects of the minor tranquilizers on sensory functioning have not been extensively investigated. Several studies indicate that $5 \mathrm{mg}$. or more of Valium will slow the rate at which neurons in the eyes, optic nerve, and brain fire, indicating that $v$ ision may be impaired (Kleinknecht \& Donaldson, 1975; Mor land, et al., 1974; Haffner, et al., 1973). This decreased sensitivity has been found to last for one and one-half hours or more following drug administration. Tests of hearing a] so indicate that Valium slows the rate at which aural neurons fire, for up to two and one-half hours after ingestion of the drug, suggesting that hearing sensitivity is decreased (Healy, et al., 1970).

Results of one study showed that the minor tranquilizers can slow the rate at which the eyes adapt to the dark for up to five hours after administration, at standard dose levels, and that the ability to accurately discriminate between objects in bright counter-light (such as the ability to see objects in the road at night even when headlights of an oncoming car are in your eyes) is also impaired. These findings suggest that minor tranquilizers produce some visual and auditory disruptions.

Vigilance performance is impaired to a greater extent than visual and auditory performance. Performance decrements are found with 5 to $10 \mathrm{mgs}$. of valium on a variety of tests such as tests of the ability to detect signals presented briefly against a background of distracting lights or sounds. Performance on tests requiring the ability to continuously scan information presented and to select a particular type of information (e.g., the letter " $\chi$ " among columns of letters or sets of four dots among clusters of any number of dots) is also impaired by Valium (Hart, et al., 1976; Wittenborn, et a1., 1979; Bernheim \& Michie1s, 1973; Clarke, et al., 1970). These findings indicate that the ability to sustain attention and concentration decreases following ingestion of minor tranquilizers even in prescribed dose levels.

The effect of minor tranquilizers on reaction time is influenced by the dose given and individual tolerance to the drug. In general, low (5 to $10 \mathrm{mg}$.$) doses do not appear to affect simple reaction time whether$ administered once only or over a period of several days (Bernheim \& Michiels, 1973; Tansella, et al., 1974; Ghoneim, et al., 1975; Ghoneim, 1975). Choice reaction time (j.e., reaction time requiring 
decisionmaking) is affected when the individual has not been taking minor tranquilizers or is given higher doses than normally prescribed. When minor tranquilizers are chronically used at low doses, no changes in either speed of reaction or number of response errors have been found (Seppala, et al., 1976; Bernheim \& Michiels, 1973; Ghoneim, et al., 1975, 1981; Landauer, et a1., 1974; Bond \& Lader, 1973).

Standard or low doses do not appear to significantly impair most motor performance. Nejther simple motor control nor more complex behaviors have been found to be impaired, as discussed below.

Speed of physical movement is not affected by low doses of minor tranquilizers. Tests of the speed with which individuals are able to tap a pencil or press a key show that subjects given minor tranquilizers were able to tap as quickly as subjects given a placebo (Bernheim \& Michiels, 1973; Milner \& Landauer, 1973; Bond \& Lader, 1973; Ghoneim, et al., 1975, 1981; Jaattela, et a1., 1976; Hart, et a1., 1976).

Tests of eye-hand coordination have also shown no minor tranquilizerinduced performance decrement. For example, when subjects were asked to maintain contact between a stylus and point on a rotating disc or to track a spot of light on a video screen with a toggle stick, their performance was unimpaired with doses of 5 to $10 \mathrm{mg}$. of Valium (Haffner, et al., 1973; Morland, et al., 1974; Kleinknecht \& Donaldson, 1975; Linnoila \& Mattila, 1973).

A review of the literature on simulated driving experiments yielded mixed results (Kleinknecht \& Donaldson, 1975). For example, Milner and Landauer (1973) administered $10 \mathrm{mg}$. Valium to subjects and found no effect on simulated driving performance. Durrman and Norman (1975) gave subjects $15 \mathrm{mg}$. Valium the day before the experiment, and one hour before the experiment, subjects were given 5,10 , or $20 \mathrm{mg}$. No significant differences were found between drugged subjects and controls.

Significant valium effects on simulated driving performance, however, have been found. Practiced subjects (i.e., subjects who are familiar with the task) were given $10 \mathrm{mg}$. Valium 30 minutes before the experiment. Drugged subjects drove faster, neglected instructions more frequently, and caused more collisions than did subjects given a placebo, but evidenced no decrease in their ability to stay on the road (Linnoila \& Hakkinen, 1974).

Minor tranquilizers appear to have their greatest effects on learning and, to some extent, memory. Substantial evidence exists to suggest that tranquilizers impair learning, but do not interfere with the ability to retrieve information from memory once it has been stored.

Studies of the effects of minor tranquilizers on short-term memory (i.e., retrieval of information presented seconds or a few minutes beforehand) indicate that these tranquilizers do not impair recall or recognition if the information was first presented when the subject was in a non-drugged state and asked to retrieve it while drugged. If the subject was under 
the influence of a tranquilizer when originally presented with words, digits, or pictures to remember, then retrieval was impaired (Ghoneim, et a1., 1981, 1975; Liljequist, et al., 1978; Ghoneim \& Mewaldt, 1975).

Long-term memory is not impaired by tranquilizers. Recall and recognition for words, digits, and pictures are unaffected by standard doses of these sedatives (Petersen \& Ghoneim, 1980; Ghonein \& Mewaldt, 1975; Mckay \& Dundee, 1980; Brown, et al., 1978).

As indicated earlier, learning is impaired by minor tranquilizers. Minor tranquilizers can induce amnesia for events as well as for words and digits (Clark, et al., 1979; Mckay \& Dundee, 1980). Subjects given tranquilizers and asked to memorize a series of digits or pairs of words require significantly more exposure to the material to learn it perfectly than subjects given a placebo (Liljequist, et al., 1978; Ghoneim, et al., 1975; Petersen \& Ghoneim, 1980). These findings suggest that tranquilizers impair the ability to store new information in memory.

\section{Barbiturates}

Although barbiturates and minor tranquilizers are chemically dissimilar, most of the effects of clinical doses of barbiturates on human performance are highly similar to the effects of larger doses of minor tranquilizers. The primary medical uses of barbiturates are to produce sedation or sleep and to prevent epileptic seizures. At therapeutic doses, the barbiturates depress the transmission of nerve impulses across the synapses in the arousal centers of the brain. At larger doses, all neurons in the body are affected and activity in the muscles, heart, and other organs of the body is decreased.

Barbiturates impair the ability to sustain attention. Results of several studies indicate that the number of errors subjects make on vigilance tasks is increased when they are drugged (Hutt, 1968; Hart, et a 1., 1976; Lehembre, 1963).

Little research has been conducted to investigate the effects of the barbiturates on perception. One study of the effects of secobarbital on smooth pursuit eye movements showed that the eyes' ability to track a moving object is disrupted at therapeutic doses (Holzman, et al., 1975). This finding suggests that the ability to monitor dials or gauges may be impaired with barbiturate use. The effects of barbiturates on the other senses, however, are unknown.

Unlike the minor tranquilizers, therapeutic doses of barbiturates slow reaction times. Studies of simple reaction time show that response speed decreases under the influence of barbiturates (Blum, Stern \& Melville, 1964; Tharp, et a1., 1974; Goldstein, et a1., 1960; Hart et a1., 1976). A study of choice reaction time found that barbiturates decreased overall reaction speed, but did not increase the number of errors the subjects made (Runde11, et al., 1978). 
As found with the minor tranquilizers, the effects of barbiturates on psychomotor performance depend upon the size of the dose administered. At the dose levels prescribed by physicians, simple motor performance is not noticeably affected (e.g., finger or toe tapping), but it is impaired when the dose level is increased (Dalton, et al., 1975; Hart, et al., 1976; Epstein and Lasagna, 1968; Klerman, et al., 1960).

More complex tests of psychomotor performance, however, show barbiturate induced performance decrements at therapeutic dose levels. Performance on tests of eye-hand coordination is impaired with barbiturates. (Klerman, et al., 1960; Billings, et al., 1975). Dalton and his associates (1975) also found barbiturate-induced decrements in manual dexterity.

Studies of the effects of barbiturates on cognitive abilities indicate that the barbiturates have a much greater effect on cognitive abilities at therapeutic dose levels than do the minor tranquilizers. Memory, learning, and reasoning abilities have all been shown to be impaired by barbiturate use.

Most studies of the effects of barbiturates on memory and learning have focused on the effects of barbiturates on short term memory, which is the ability to retain information for seconds or minutes after it is presented. The ability to recall word lists and lists of digits decreases with therapeutic doses of barbiturates, as does the ability to recognize words previously presented from among lists of similar words presented as distractors (Runde11, et al., 1978; Evans \& Davis, 1969; Hurst, et al., 1968; Malpas \& Joyce, 1969; Hart, et a1., 1976; Adams, 1974; Epstein \& Lagasna, 1968; Hutt, et a1., 1968; Blum, et a1., 1964).

Barbiturate effects on long-term memory, or the ability to retain information for hours, days or longer, have received much less attention in the literature. Results of one series of studies (Rundell, et al., 1978) indicate that long-term memory is adversely affected by barbiturates. These researchers found that the ability to recognize words presented several hours earlier from among a list of distractors was impaired by secobarbital, a short-acting barbiturate. The ability to search memory for items of information was not reduced by secobarbital, but the experimental subjects were less able to differentiate the actual words previously presented from distractors than were subjects given a. placebo. This finding suggests that the words were inaccurately or incompletely stored in memory by the subjects when they first were presented.

Results of several studies of the effects of barbiturates on reasoning indicate that the ability to solve problems is impaired by therapeutic doses of these drugs. A number of researchers have found that the ability to perform arithmetic operations is decreased with barbiturates (Epstein \& Lasagna, 1968; Klerman, et al., 1960; Blum, et al., 1964). The time required by the experimental subjects to solve the arithmetic problems increased as did the number of their errors. 
Other studies of barbiturate effects on higher mental functioning have primarily been conducted with persons who have epilepsy or other seizure disorders. Since repeated seizures are thought to produce permanent brain damage and to impair congitive functioning, it is difficult to separate the effects of the convulsions from the effects of anticonvulsant drugs on the reasoning abilities of persons who both take the drugs and suffer from seizure disorders. Studies of persons who take barbiturates but who have had few seizures, or studies which attempt to separate drug effects from seizure effects with statistical techniques have found deterioration in cognitive functioning associated with long-term use of barbiturates (Thompson, et al., 1980). Both overall school performance in children and performance on IQ tests of children and adults have been shown to decrease as blood levels of the barbiturates increase (Trimble \& Corbett, 1978; Reynolds \& Travers, 1974). Because these studies were conducted with special populations and the researchers could not control the doses of barbiturates given, it cannot be concluded that the barbiturates caused the deterioration noted or that these same effects would be found with other subjects. These findings do suggest, however, that therapeutic doses of barbiturates may impair reasoning abilities.

Barbiturates aiso affect communicative skills. Persons who have been given therapeutic doses of barbiturates both speak less in a social situation and speak slower than persons given a placebo. The drugged subjects' thought patterns, as demonstrated by the content of their speech, were unaffected, however, (Hutt, et al. 1968; Stitzer, et al., 1981). These findings suggest that performance at any job which requires communication skills will be impaired.

Summary

To summarize, sedatives when taken at standard dose levels are found to produce the following effects:

- Performance decrements due to sedative ingestion have been found for perceptual/sensory tasks; auditory and visual capacity is less sensitive to input; however, the most pronounced effect found is for performance decrements in sustaining attention and concentrating on tasks requiring vigilance.

- Some types of motor performance are affected by sedatives (i.e., choice reaction time) but performance decrements disappear when sedatives are chronically used; other motor skills such as eye-hand coordination, complex driving tasks, etc., show no decrements due to low doses of minor tranquilizers, but are impaired by barbiturates. 
- Cognitive performance, in particular the learning of new material, is impaired; when learning occurs in a sedated state, subjects show significant impairment in their ability to evidence the learning and to retrieve or recall the information at a later point in $t$ ime.

- Communicative sikills are not likely to be adversely affected by low doses of minor tranquilizers, but are impaired due to barbiturates.

\section{Interactive Effects of Sedatives}

To examine the interactive effects of sedatives, some further discussion of the effects of sedatives on the central nervous system is useful. Their effects are dose-dependent, with a progression with increasing dose as follows: anxiety reduction $\rightarrow$ disinhibition $\rightarrow$ sedation $\rightarrow$ hypnosis (sleep) $\rightarrow$ general anesthesia $\rightarrow$ coma $\rightarrow$ death (Julien, 1981). They are most effective when their use is time-limited (i.e., used for 1 to 8 weeks and then stopped until symptoms recur) (Julien, 1981). Nonetheless, it has been estimated that two million persons in the United States take Valium(B) continuously (Jick, 1974, cited in Linnoila, 1976).

When combined with other central nervous system depressants such as alcohol or other drugs within the class of sedatives, sedatives are referred to as having "potentiating" effects rather than being simply additive as with marjjuana and alcohol. The significance of the potentiating interaction of sedatives has greatest bearing on multiple substance abuse. For example, the individual who has a standard dose of a sedative and then a drink or two at lunch will experience an effect that is greater than the simple addition of one unit of effect due to the sedative, plus two units of effect due to the alcohol. (The unpredictability of one sedative in particular, Valium (B) has been noted; the interactive effects are so unpredictable that the combination of one drink and one standard dose has been known to cause death.*

Performance decrements due to sedative interaction effects have not been covered in the scientific literature. It is likely, however, that the effects on performance noted above would become more severe when two different sedatives are taken together. Also, the course of events outlined above clearly suggests that attention and learning would be significantly impaired with increasing doses of sedatives as individuals progress toward a hypnotic condition.

*Rose, Mitche11. Lecture given for Association for Advanced Training in the Behavioral Sciences, Los Angeles, California, March 1982. 
HALLUCINOGENS

Hallucinogens consist of a variety of drugs that distort sensory perceptions, thought processes, and behavior. It is thought that these drugs operate on, and highly resemble, naturally occurring chemicals in the brain (Julien, 1981). It is expected that this class of drugs will be used on the job only rarely; consequently, the review of their effects on performance is limited in scope and detail.

Four groups of hallucinogens have been identified on the bas is of their effect on particular chemical substances within the brain. The first of these, the "anticholinergics" (e.g., atropine, scopolamine, malathion) are highly toxic and produce severe side effects. Many insecticides such as malathion are rarely used except by seriously debilitated drug abusers. Consequently, they are not addressed here, since few of the abusers are likely to be employed.

The second group of hallucinogens, the catechols, includes peyote, mescaline, and synthetic mescaline agents such as MDA and MMDA. The most pronounced effects associated with the use of these substances are the marked distortions in perception of light, color, space, and shapes. Users are typically alert and give no evidence of memory loss. Ingestion of large doses (i.e., 200 to $500 \mathrm{mg}$. for mescaline), however, may lead to severe muscle spasms (Julien, 1981).

Third, the indole group consists of LSO, psilocybin, and morning glory seeds (ololiuqui). While users seldom experience serious physical effects, they frequently encounter dramatic changes in mood and sensory perception (Gaston \& Waiker, 1981). Actual effects are apt to vary, depending on the user's expectations, amount of drug ingested, personality, and setting. In general, effects include an aitered sense of time, space, touch, color, and blurred vision and hearing. With higher doses (i.e., 5 to $15 \mathrm{mg}$. for psilocybin and 200 to $500 \mathrm{mg}$. for LSD), one experiences mood fluctuations (euphoria, fear, hostility), problems in speaking clearly, visual hatlucinations, confusion, and impairment of thought processes. A decline in motor function and increased sleepiness are also linked with psilocybin use. Although rare and unpredictable, flashbacks may occur up to one year after use of LSD.

Finally, use of the hallucinatory anaesthetics (the most common being PCP) is linked with general confusion, spatial disorientation, aggressiveness, and feelings that one has great physical strength or that one is about to die (Gaston \& Wa $7 k e r, 1981$ ). The user may experience trouble breathing and, at higher doses, become unconscious. There is, in addition, little sense of pain. Although long-term and behavioral effects are quite unpredictable, the anaesthetics are considered to be the most dangerous of hallucinogens, possibly resulting in permanent brain damage.

Overall, the occasional use of hallucinogens has not been shown to result in physical addiction, chromosomal damage, anti-social behavior, or 
long-term psychological disorientation (Gaston \& Walker, 1981). Use of hallucinogens on the job, however, presents a severe safety danger and would preclude effective job performance for any employee. 
Appendix D

Impairing Effects of Alcohol on Human Performance

Alcohol has been found to impair performance on a wide variety of tasks, ranging from simple motor reflex responses to higher-level

problem-solving. Early inconsistencies in the experimental literature suggesting that large doses of alcohol improve performance have been set to rest. It is now accepted that alcohol produces performance decrements, some of which begin to appear at very low doses.

About $95 \%$ of the alcohol consumed is metabolized in the liver where it is changed to water and carbon dioxide before excretion. The rate at which alcohol is metabolized is slow and constant: about one-third ounce of pure ethanol per hour in the average adult. This metabolic rate is unaffected by the amount consumed, blood alcohol concentrations (BAC), or the consumption of food or other ilquids (Wallgren \& Barry, 1970; AMA Committee on Medicolegal Problems, 1970; Jones, 1978; Julien, 1981).

Like the sedatives, alcohol is a central nervous system depressant. At low doses, alcohol creates mild euphoria and behavioral excitement. This response has been interpreted by some as evidence that inhibitory synapses are depressed before excitatory ones, creating the state of disinhibition and arousal commonly noted after drinking begins (Julien, 1981; Perrine, 1974). With increasing doses, behavioral activity is progressively reduced to the point of coma or death.

Individual differences affect the onset of behavioral disruption due to alcohol consumption. Greater body weight, a slow speed of consumption, and physical tolerance to alcohol raise the $B A C$ at which substantial behavioral disruption occurs. In general, however, disruption in human performance occurs at relatively low doses. Some behavior, for example, the ability to perceive movement (Jellinek \& McFarland, 1940; Levine, Greenbaum \& Notkin, 1973; Jones, 1978) is affected after as few as two drinks ( $B A C=.03 \%$ ) in the average American male, who weighs 172 pounds (The National Observer, 1976). In general, motor behaviors are impaired before cognitive abilities and recover later than cognitive skills, as the blood alcohol concentration rises, then falls, following alcohol ingestion. In the following discussion, $B A C$ will be discussed in terms of the number of drinks required for the average American male.

The impact of alcohol on sensory/perceptual performance will be discussed first. Alcohol affects some visual capacities after one to two drinks and continues even after all alcohol has been removed from the body. other visual and sensory/perceptual abilities are unaffected until high doses of alcohol.

Accurate perception of motion is impaired at low alcohol doses. Alcohol reliably induces positional nystagmus, or rapid and uncontrollable oscijlations of the eye, as early as 15 minutes after ingestion and the nystagmus lasts up to 18 hours after the first drink is taken (Jones, 1978; Carpenter, 1962). Alcohol-induced nystagmus interferes with the 
ability to fix and maintain visual focus on any moving target (Levy, et a]., 1980).

The threshold at which a rapidly blinking light is seen as a steady light decreases with alcohol ingestion; perception of rapjdly changing events in a visual field may be impaired (Enzer, et al., 1944; Goldberg, 1943). Further, the ability to distinguish close, but separated, moving objects is impaired after one to two drinks (Honneger, Kampschulte \& Klein, 1970). Visual acuity for unchanging events is not significantly affected until after at least four to five drinks. Distinguishing close but separated, unmoving objects is affected after less than four to five drinks (Newman \& Fletcher, 1941; Mortimer, 1963).

Discriminating different light intensities is impaired significantly at low (one to two drinks) and moderate (four to five drinks) doses (Carpenter, 1962). However, depth perception, peripheral vision, perception of colors, and dark adaptation are not affected until six to seven drinks are consumed (Zwahlen, 1976; Lewis, Dustman \& Beck, 1969; Lewis, 1972; Moskowitz, 1974; Newman \& Fletcher, 1941; Carpenter, 1962).

Substantial doses of alcohol are necessary to significantly impair auditory and olfactory perception (Jellinek \& McFarland, 1940; Carpenter, 1962). Alcohol, as a painkiller (analgesic), has been found to reduce sensitivity to pain, but not decrease sensitivity to touch.

Attention to simple auditory and visual vigilance tasks is unaffected by alcohol (Talland, et al., 1964; Moskowitz \& DePry, 1968; Pearson \& Neal, 1970). Vigilance performance on tasks requiring divided attention is impaired after four to five drinks (Moskowitz \& Burns, 1971; Moskowitz \& DePry, 1968; Moskowitz \& Roth, 1971). Divided attention tasks require the subject to discriminate between incoming stimuli and to respond differentially to the stimuli. Other visual vigilance tasks have shown significant performance decrements because of alcohol-induced drowsiness (Erwin, et a)., 1978).

In general, then, studies of attention and perception suggest that performance on tasks which require perceptual acuity and simple sustained attention are not significantly reduced with low and moderate doses of alcohol. The ability to monitor continuously and respond to rapidly changing events will begin to deteriorate at low doses, however. After six drinks have been consumed, performance on even simple perception and attention tasks is impaired.

Increases in simple reaction time are found consistently with alcohol ingestion (Carpenter, 1962; Jellinek \& McF arland, 1940; Taberner, 1980; Linnoila, et a .., 1978; Zwahlen, 1976). After four to five drinks, response speed to visual stimuli slows; response time to peripheral visual stimuli is impaired with even fewer drinks (Carpenter, 1962; Lubin, 1977). Auditory simple reaction time is similarly impaired (Dettling, 1956; Forbes, 1947; Howells, 1956). Reaction time decrements are intensified by increasing alcohol consumption. Greater impairment of 
reaction time occurs when blood alcohol concentration is increasing than when the blood alcohol concentration is decreasing (Young, 1970).

$V$ isual and auditory choice reaction time (i.e., requires decision with regard to response to two or more stimuli) increases with alcohol; accuracy of response significantly decreases with continued drinking (i.e., the blood alcohol concentration is rising) (Linnoila, et al., 1978; Jones, 1978; Runde11, et al., 1978; Pearson \& Nea 1, 1970; Tharp, et al., 1974). The speed required to make a correct response is slowed more than the speed with which incorrect responses are made (Jennings, et al., 1976; Rundell \& Williams, 1979). These increased reaction times occur after three to four drinks.

Alcohol affects simple motor responses after three to four drinks. Nearly all individuals demonstrate significant decreases in standing steadiness after six to seven drinks (Jones, 1978). Simple finger and toe tapping speed also decreases (Carpenter, 1962).

Eye-hand coordination is severely disrupted by alcohol after six or seven drinks. Handwriting clarity, error-free typing, and target shooting are all impaired by alcohol intoxication, although typing of an over learned passage was only impaired when blood alcohol concentrations were rising rather than falling (Rabin \& Blair, 1953; Schweitzer, 1955; Prag, 1953; Eggleton, 1941; Newman, 1947; Newman \& Abramson, 1941).

Much research on motor performance investigates the effects of alcohol on tracking tasks. Tracking tasks require the subject to track manually a moving target by manipulating a stylus or toggle switch, a skill necessary for successful Jriving and success at many video games. Performance on simple tracking tasks is not impaired at low doses of alcohol, or fewer than three drinks. Time on target decreases significantly with consumption above three drinks and as the complexity of the task increases (Dott \& Mckelvy, 1977; Vogel-Sprott, 1979; Pearson \& Neal, 1970; Klein \& Jex, 1975). As opposed to performance decrements found in perception and reaction time, performance on tracking tasks is unaffected by whether blood alcohol concentrations are rising or falling (Klein \& Jex, 1975; Vogel-Sprott, 1979), but greater impairment has been noted when blood alcohol concentration is rapidly rising rather than increasing at a slower rate (Eggleton, 1941).

Driving behavior is a complex motor skill requiring significant cognitive input. The ability to respond with accurate and cognitively integrated actions is affected by alcohol consumption. Driving skills deteriorate after three to four drinks and sharply decline after four to five drinks. Impairment of performance on driving simulator tests shows after three to four drinks (Drew, et al., 1958; Loomis \& West, 1958; Stening \& Dureman, 1974). Real car driving at slow speeds on closed courses shows that backing up, steering accuracy, and parking skills are disrupted after one or two drinks (Bjerver \& Goldberg, 1950; Hunt ley \& Perrine, 1971; Lovibond \& Bird, 1970). Degraded performance is consistently demonstrated on closed course driving after three to four drinks (Coldwe11, et al., 1958; Longhetti \& Barnett, 1965). Response to 
emergencies shows increased emergency braking distances and significant impairment of steering accuracy after two to four drinks (Laurell, 1977).

Investigations of industrial tasks show the effects of moderate amounts of alcohol. Performance on a production assembly task of water taps shows that assembiy time increased and quality of work decreased significantly after five to six drinks (Price \& Hicks, 1979). The effect of six to seven drinks was evaluated on performance of three elements of an arc-welding task: (1) speed of electrode movement, (2) angle of electrode, and (3) electrical current reversals due to changes in the size of the gap between the electrode and the material used. Only stability in the maintenance of the electrode/material gap was impaired (Price \& Liddle, 1982). Price and Flax (1982) also investigated the effects of two, three to four, and five to six drinks on the operation of a drill press. One drink showed no performance impairment, but the number of accurate hits decreased by $12 \%$ and $19 \%$ after three to four and five to six drinks, respectively.

The effects of alcohol on memory and learning are complex. Alcohol can enhance or impair memory, depending upon the blood alcohol concentration and whether it is rising or falling, the state (either intoxicated or sober) of the subject at the time of learning or performance of the memory task, and the type of task employed.

Small doses of alcohol may facilitate memory. Alcohol, given after material is learned, appears to facilitate consolidation of memory traces. Recognition of material presented improves after two to four drinks (Parker, et al., 1981, 1980). Most other studies of a]cohol and memory, however, show that alcohol disrupts memory processes. Alcohol produces performance deficits in recall and recognition tasks for both short-term and long-term memory.

Short-term memory of verbal and visual information is impaired after five drinks (Birnbaum \& Parker, 1977; Ryback, 1977; Craik, 1977; Runde 11 \& Williams, 1977), though it is unaffected by rising versus falling blood alcohol concentration (Jones \& Jones, 1977). A more pronounced effect occurs when complex material is involved (Weingartner \& Murphy, 1977; Craik, 1977).

Retrieval of information from long-term memory is disrupted by alcohol. Free recall and recognition performance are impaired when learning and retrieval occur under the influence of alcohol (Runde11, et a1., 1978). Cued recall tests show that retrieval deficits can be overcome if cues are given to intoxicated subjects (Poulos, et al., 1981; Birnbaum \& Parker, 1977). These findings suggest that the information has been stored in the memories of intoxicated subjects, but that alcohol interferes with their ability to "locate" it.

Memory processes with alcohol are state-dependent, i.e., depend on what state (sober or intoxicated) the subject is in when learning or retrieving information. A review of the literature on state-dependent learning and memory (Eich, 1977) notes the following: 
- Retrieval is more impaired when material is learned in one state and tested in another, than when it is learned and tested in the same state.

- Moderate doses of alcohol (five to six drinks) produce the largest state-dependence effects on learning or test performance.

- Information transfers better when learned sober and retrieved under the influence of alcohol than when learned intoxicated and retrieved sober.

- Tasks which require some cognitive processing or organization and which require the use of order information are most subject to state-dependence effects.

However, while retrieval of information can be somewhat improved if an intoxicated person originally acquired the information when intoxicated, retrieval deficits due to alcohol will still be found when comparing sober and intoxicated individuals.

Alcohol's most significant effect on memory and learning appears to be on the abjlity to organize new information in long-term memory when under the influence of alcohol. Long-term memory refers to information to be remembered for hours, days, or longer. Information is better retained when organized into meaningful clusters than when the items are unrelated, e.g., "2-4-6-8" versus "1-15-12-4." With repeated exposure to information, sober persons impose their own organization on even unrelated items, and retention improves (Murdock, 1974). Alcohol consumption of six or more drinks reduces the ability to organize information significantly (Rundell, et al., 1978; Rundell \& Williams, 1977; Parker, et al., 1974). Consequently, the amount of new information an intoxicated person will retain is decreased and the number of exposures to the information necessary to learn it will increase (Rundell \& Williams, 1977).

To conclude, these studies of learning and memory suggest that retrieval of even well-learned information may not be possible for persons performing under the influence of alcohol. Efficiency is reduced when time is spent looking for misplaced papers or tools; the proper sequence for carrying out standard tasks can be forgotten, or recollection of which tasks are complete and which are not can be impaired. Finally, alcohol is particularly likely to interfere with learning and retaining complex information.

Higher-order problem-solving abilities are more resistant to alcohol's effects than simpler cognitive and motor processes. Greater alcohol consumption is necessary to produce impairments in reasoning and normal functioning is restored more quickly once the BAC begins to fall, than it is for simpler tasks. Complex problentsolving ability does deteriorate with moderate doses of alcohol, however, and when considered in conjunction with the learning and memory research, this suggests that 
problem-solving ability and the ability to think in an ordered, logical manner may be adversely affected by alcohol.

Alcohol effects on reasoning tasks depend on the type of task involved and whether blood alcohol concentrations are rising or falling. For example, numerical reasoning is impaired with smali doses of alcohol. Mental addition, subtraction, and division tasks are impaired after three drinks; more errors are made and performance is slowed also (Goldberg, 1943; Takala, et a1., 1958; Duker, 1956; Zirkle, et a .., 1959; Zirkle, et al., 1960; Frankenhaueser, et al., 1962; Lewis, et al., 1969).

Verbal reasoning and abstraction is less impaired at low doses than numerical reasoning. Performance decrements do show after six to seven drinks, and are only found when blood alcohol levels are rising. Even after six or more drinks, if the $B A C$ is falling, verbal reasoning and the ability to form and use abstract concepts are not impaired (Jones \& Vega, 1972; Pohl, 1978; Frankenhaueser, et al., 1962).

A study of linear problem-solving, requiring the use of a mathematical language to solve logic problems, shows low doses (three to four drinks) facilitate performance; higher doses (six to seven drinks) impair performance. Alcohol does not interfere with the correct use of mathematical language, but at the higher doses, problem-solving efficiency is reduced.

Another aspect of cognitive performance, judgment, shows performance decrements due to alcohol in two areas: willingness to take risks, and judgments about one's own or others' degree of alcohol-induced impairment. Intoxicated individuals are willing to take greater risks than sober persons. Alcohol increases the willingness to take risks in card games or other wagering situations (wallgren \& Barry, 1970). Alcohol impairs passing judgment on tests of driving skill (Light \& Keiper, 1971), and increases acceptance of risk in other types of driving situations (Cohen \& Hansel 1958; Linnoila \& Mattila, 1973; Lewis \& Sarlanis, 1969). Inexperienced drinkers are more likely to take increased risks when intoxicated than experienced drinkers (Goodwin, Powell \& Stein, 1973). The above studies look at risk-taking effects after six to seven drinks.

Alcohol also impairs the ability to judge accurately the effects of alcohol on self-awareness and judgments of others. Judgments of one's own response speed on a reaction time task is increasingly inaccurate after four to six drinks. Self-awareness of performance is diminished also (Lubin, 1977; 1979). Further, intoxicated subjects are also less able to judge accurately others' performance levels and their degree of intoxication. Inexperienced drinkers are more impaired on these judgment tasks than experienced drinkers (Lubin, 1979).

The findings of inaccurate judgment and greater willingness to risk suggest that under the influence of alcohol individuals may feel comfortable in violating procedures or be less cautious in their behavior, in general. Also, evidence of reduced self-awareness when 
moderately intoxicated suggests that individuals may not be aware of their performance decrements.

The peak effects of an alcohol/sedative combination occur with in the first hour after both are ingested. Some evidence exists to suggest that alcohol accelerates the absorption rate of sedatives (Linnoila \& Mattila, 1973). Even if sedatives are taken up to ten hours before alcohol is consumed, effects of the combination are still observable (Linnoila, 1976).

Alcohol/sedative combinations significantly impair motor performance. Eye-hand coordination is decreased and reaction time greatly increased under the influence of low doses of these two drugs (Linnoila \& Mattila, 1973). In studies of simulated driving, subjects taking alcohol and a sedative were involved in more collisions, drove off the road more frequently, and ignored driving rules more often than persons taking either substance alone (Linnoila \& Haakkinen, 1974; Linnoila \& Mattila, 1973). Alcohol/sedative combinations also affect learning and memory. In particular, they disrupt short-term memory and performance on simple learning tasks (Liljequist, et al., 1975).

Alcohol and sedatives are said to potentiate each other. As discussed in the sedative section (Section 5.5), potentiating interactions occur when the effects of dose combinations are more than simply additive. Therefore, with even small doses of both alcohol and sedatives taken in combination the behavioral effects include unsteadiness, disorientation, impaired judgment of intoxication, deficits in attention, and motor performance capabilities.

Different effects are found for alcohol/marijuana combinations. Marijuana, though not a central nervous system depressant, induces behavior effects similar to those found with alcohol (Julien, 1981). Unlike the alcohol/sedative combination, marijuana and alcohol do not combine synergistically, i.e., consumption of marijuana will not potentiate alcohol's action or vice versa. They do, however, impair performance to a greater extent than if either alcohol or marijuana is consumed alone. The combination particularly affects motor performance. Several studies note substantial decreases in standing steadiness and eye-hand coordination, and increased reaction times due to the combination (Bird, et a 1., 1980; Manno, et a1., 1971; Reid, et a1., 1972; Macavoy \& Marks, 1975).

Cognitive performance is also affected by alcohol and marijuana taken together. Numerical reasoning, or the ability to add and subtract quickly without errors, is debilitated by the combination (Chesher, et al., 1977; Belgrave, et al., 1979b). In general, the combination of these two drugs significantly affects all aspects of human performance. Any ability which is negatively impacted by ejther drug alone will also be impaired by the combination.

Long-term use of moderate amounts of alcohol (up to two ounces of pure ethanol per day) produces few permanent physical or behavioral changes. 
Long-term use of large amounts of alcohol is both physically and psychologically debilitating. Disorders associated with chronic alcohol abuse include dysfunctions of the heart, liver, gastrointestinal tract; a predisposition toward cancer; brain damage; and apparent permanent impairment of the higher mental functions, such as memory, reasoning, and judgment. Chronic alcohol abuse is characterized by tolerance to the effects of alcohol and the development of physical dependence upon it. Tolerance occurs with regular ingestion of large amounts. Increasing doses are required to produce the physical and behavioral effects of acute intoxication. Physical dependence occurs when withdrawal of a)cohol produces motor tremors, a state of hyperexcitability, sleep disorders, confusion and disorientation, and sometimes convulsions and death. 


\section{Appendix $E$}

Bibliography

Abel, E.L. 1971. "Retrieval of Information After Use of Marijuana." Nature 58:231.

Abelson, H.I. and R.B. Atkinson. 1975. Public Experience with Psychoactive Substances. Response Analys is Corporation, Princeton, New Jersey.

Abelson, H.I. and P.M. Fishburne. 1976. Nonmedical Use of Psychoactive Substances. Part I: Main Findings. Response Analysis Corporation, Prínceton, New Jersey.

Adams, R.G. 1974. "Pre-Sleep Ingestion of Two Hypnotic Drugs and Subsequent Performance." Psychopharmacologia 40:185-190.

American Medical Association Committee on Medicolegal Problems. 1970. Alcohol and the Impaired Driver: A Manual on the Medicolegal Aspects of Chemical Tests for Intoxication With Supplement on Breath/A cohol Tests. National Safety Council, Chicago.

Babor, T.F., J.H. Mendelson, and J. Kuehnle. 1976. "Marihuana and Human Physical Activity." Psychopharmacology 50:11-19.

Baird, E. and D. Hailey. 1972. "Delayed Recovery from a Sedative: Correlation of the Plasma Levels of Diazepam with Clinical Effects After Oral and Intravenous Administration." British Journal of Anaes thes iology $44: 803-808$.

Barmack, J. 1940. American Journal of Psychiatry 97:163.

Bech, P., L. Rafaelsen, and 0.J. Rafaelsen. 1973. "Cannabis and Alcohol: Effects on Estimation of Time and Distance." Psychopharmacologia 32:373-381.

Bejerot, N. 1970. "A Comparison of the Effects of Cocaine and Synthetic Central Stimulants." British Journal of Addiction $65: 35-37$.

Belgrave, B.E., et al. 1979a. "The Effect of Cannabidiol Alone and in Combination with Ethanol, on Human Performance." Psychopharmacology $64: 243-246$.

Belgrave, B.E., et al. 1979b. "The Effect of Trans-Delta 9Tetrahydrocannabinol, Alone and in Combination with Ethanol, on Human Performance." Psychopharmacology 62:53-60.

Bensinger, P.B. 1982. "Drugs in the Workplace." Harvard Business Review Nov-Dec:49-60. 
Bernheim, J. and W. Michiels. 1973. "Effets Psychophysiques du Diazepam (Va)ium) et d'une faible dose d'alcool chez 1'homme." Schweiz. Med. Wochenschr. 103:863-870.

Billings, C., R. Gerke, and R. Wick. 1975. "Comparisons of Pilot Performance in Simulated and Actual Flight." Aviation, Space and Environmental Medicine 46:304-308.

Bird, K.D., et al. 1980. "Intercannabinoid and Cannabinoid-Ethanol Interactions and Their Effects on Human Performance." Psychopharmacology 71:181-188.

Birnbaum, I.M. and E.S. Parker. 1977. "Acute Effects of Alcohol on Storage and Retrieval." In Alcohol and Human Memory, eds. I.M. Birnbaum and E.S. Parker, pp. 99-108. ErTbaum, HiTTsdale, New Jersey.

Bjerver, K. and L. Goldberg. 1950. "Effects of Alcohol Ingestion on Driving Ability: Results of Practical Road Tests and Laboratory Experiments." Quarterly Journal of Studies on Alcohol 11:1-30.

Blum, B., M.H. Stern, and K.I. Melville. 1964. "A Comparative Evaluation of the Action of Depressant and 5timulant Drugs on Human Performance." Psychopharmacologia 6:173-177.

Blum, K. 1976. "Depressive States Induced by Drugs of Abuse: ClinicaT Evidence, Theoretical Mechanisms and Proposed Treatment, Part II." Journal of Psychedelic Drugs $B(3): 235-262$.

Boe, S. 1973. "How the Pharmaceutical Manufacturer Guards Against Drug Abusers." In Drug Abuse in Industry, ed. J.M. Scher. Charles C. Thomas, Springfield, ITlinois.

Bond, A. and M. Lader. 1973. "The Residual Effects of Flurazepam." Psychopharmacologia 32:223-235.

Borg, J., S. Gershon, and M. Alpert. 1975. "Dose Effects of Smoked Marihuana on Human Cognitive and Motor Functions." Psychopharmacologia 42:211-218.

Bouquet, J. 1944. "Marjhuana Intoxication." (Letter) Journal of the American Medical Association 124:1010-1011.

Bowman, M. and R.0. Pihl. 1973. "Cannabis: Psychological Effects of Chronic Heavy Use." Psychopharmacologia 29:159-170.

Braff, D.L., et aT. 1981. "Impaired Speed of Visual Information Processing in Marijuana Intoxication." American Journal of Psychiatry 138(5):613-617.

Brewin, R. 1978. "Businessmen Hooked on Valium." Dun's Review $11: 44-46$. 
Brown, J., et al. 1978. "Amnesic Effects of Intravenous Diazepam and Lorazepam." Experientia 34:501-502.

Buikhuisen, W. and R.W. Jongman. 1972. "Traffic Perception under the Influence of Alcohol." Quarterly Journal of Studies on Alcohol $33: 800-806$.

Cahalan, 0. 1970. Problem Drinkers. Jossey-Bass, San Francisco, California.

Cahalan, D. 1978. "Implications of American Orinking Practices and Attitudes for Prevention and Treatment of Alcoholism." In Behavioral Approaches to Alcoholism, eds. G. Marlatt and P. Nathan. Rutgers Center of Alcohol Studies, New Brunswick, New Jersey.

Cahalan, D. and R. Room. 1972. Problem Drinking Among American Men. Monograph No. 7. Rutgers Center of ATcohol Studies, New Brunswick.

Cahalan, D., I.H. Cisin, and H.M. Crossley. 1969. American Drinking Practices: A National Study of Drinking Behavior and Attitudes. Rutgers Center of Alcohol Studies, New Brunswick, New Jersey.

Caldwell, D.F., et al. 1969. "Auditory and Visual Threshold Effects of Marijuana in Man." Perceptual and Motor Skills 29:755-759.

Campbell, J.P., et a1. 1970. Managerial Behavior, Performance, and Effectiveness. McGraw-Hill, New York.

Carlin, A.S. and R.D. Post. 1971. "Patterns of Drug Use Among Marihuana Smokers." Journal of the American Medical Association 218:867-868.

Carpenter, J.A. 1962. "Effects of Alcohol on Some Psychological Processes: A Critical Review with Special Reference to Automobile Driving Skill." Journal of Studies on Alcohol 23:274-314.

Carpenter, J.A., et al. 1961. "Alcohol and Higher-Order Problem Solving." Quarterly Journal of Studies on Alcohol 22:183-222.

Carroll, J., et al. 1977. "The Meaning and Evolution of the Term 'Multiple Substance Abuse'." Contemporary Drug Problems Summer, 1977, pp. 101-133.

Casswe11, S. 1975. "Cannabis Intoxication: Effects of Monetary Incentive on Performance, a Controlled Investigation of Behavioural Tolerance in Moderate Users of Cannabis." Perceptual and Motor Skills $41(2): 423-434$.

Casswe 11, S. and D. Marks. 1973. "Cannabis Induced Impairment of Performance of a Divided Attention Task." Nature 241:60-61. 
Chambers, C.D. 1971. Differential Drug Use Within the New York State Labor Force. New York State Narcotic Addiction Control Comission. Cited from a reference In Drug Abuse: Clinical and Basic Aspects, eds. S.N. Pradhan and S.N. Dutta. C.V. Mosby Co., St. Louis, 1977.

Chambers, C.D. and R.D. Heckman, 1972. Employee Drug Abuse: a Manager's Guide for Action. Chaners Publishing Co., Inc., Boston, Massachusetts.

Chambers, C.D. and J.A. Inciardi. 197la. An Assessment of Orug Use in the General Population. New York State Narcotic Addiction Controt Comission. Cited from a reference In Drug Abuse: Clinical and Basic Aspects, eds. S.N. Pradhan and S.N. Dutta. C.V. Mosby, St. Louis, 1977.

Chambers, C.D. and J.A. Inciardi. 1971b. An Assessment of Drug Use in the General Population. Special Report No. 2. New York State Narcotic Addiction Controt Commission. Cited from a reference In Drug Abuse: Clinical and Basic Aspects, eds. S.N. Pradhan and S.N. Dutta. C.V. Mosby Co., St. Louís, 1977 .

Chambers, C.D., J.A. Inciardi, and H.A. Siegal. 1975. Chemical Coping. Spectrum Publications, New York.

Charen, S. and L. Perelman. 1946. "Personality Studies of Marihuana Addicts." American Journal of Psychiatry 102:674-682.

Chesher, G.B., et al. 1977. "Ethanol and Delta-9-Tetrahydrocannabinol Interactive Effects on Human Perceptual, Cognitive and Motor Functions." Medical Journal of Australia 641:478-481.

Chopra, I.C. and R.N. Chopra. 1957. "The Use of the Cannab is Drugs in India." United Nations Bullet in on Narcotics 9:4-29.

Church, M. and L. Johnson. 1979. "Mood and Performance of Poor Sleepers During Repeated Use of Flurazepam." Psychopharmacology 61:309-316.

Clark, E., M. Glanzer, and H. Turndorf. 1979. "The Pattern of Memory Loss Resulting from Intravenously Administered Diazepan." Archives of Neurology $36: 296-300$.

Clark, L.D. and E.D. Nakashima. 1968. "Experimental Studies of Marihuana." American Journal of Psychiatry $125(3): 379-384$.

Clark, W.B., et a1. 1981. Report on the 1979 National Survey. Social Research Group, University of California, BerkeTey, California.

Clarke, P., et a1. 1970. "The Amnesic Effect of Diazepam (Valium)." British Journal of Anaesthes jology 42:690-697.

Clopton, P.L., et a]. 1979. "Marijuana and the Perception of Affect." Psychopharmacology 61:203-206. 
Cohen, J. and C.E.M. Hanse1. 1958. "The Risk Taken in Driving Under the Influence of Alcohol." British Medical Journal 1:1438-1442.

Coldwell, B.B., et al. 1958. "Effect of Ingestion of Distilled Spirits on Automobile Driving Skill." Quarterly Journal of Studies on Alcohol 19:590-616.

Craik, F.I.M. 1977. "Similarities Between the Effects of Aging and Alcoholic Intoxication on Memory Performance, Construed Within a 'Levels of Processing' Framework." In Alcohol and Human Memory, eds. I.M. Birnbaum and E.S. Parker, pp. 9-21. ErTbaum, HilTsdale, New Jersey.

Crancer, A., Jr. 1969. "Marihuana and Simulated Driving." Science $166: 640$.

Dalton, W.S., et al. 1975. "Effects of Marjhuana Combined with Secobarbital." Clinical Pharmacology and Therapeutics 18(3):298-304.

Danielson, L.E. 1960. Characteristics of Engineers and Scientists. University of Michigan, Bureau of Industrial Relations, Ann Arbor, Michigan.

Darley, C.F., et al. 1977. Marijuana Effects on Long-Term Memory Assessment and Retrieval." Psychopharmacology 52:239-24 I.

Darley, C.F., et al. 1974. "The Nature of Storage Deficits and StateDependent Retrieval under Marihuana." Psychopharmacologia 37:139-149.

Darley, C.F., et a1. 1973. "Marihuana and Retrieval from Short-Term Memory." Psychopharmacologia 29:23l-238.

Darley, C.F. and J.R. Tink lenberg. 1974. "Marijuana and Memory." In Marijuana: Effects on Human Behavior, ed. L.L. Miller. Academic Press, New York.

Demarest, M., et a1. 1981. "Cocaine: Middle Class High." Time $118: 56-60+$.

Deneau, G.A. and S. Kaymakcalan. 1971. "Physiological and Psychological Dependence to Synthetic Delta-9-Tetrahydrocannabinol (THC) in Rhesus Monkeys." The Pharmacologist 13:246.

Dettling, $L$. 1956. "L'influence de 1'alcohol sur le temps de reaction d'un automobiliste." Review of Alcohol 4:40-45.

Dittrich, A., K. Battig, and 1. von Zeppelin. 1973. "Effects of Delta-9-Trans-Tetrahydrocannabinol (THC) on Memory, Attention and Subjective State: a Double Blind Study." Psychopharmacologia 33:369-376. 
Dornbush, R.L. 1974. "Marijuana and Memory: Effects of Smoking on Storage." Annals of the New York Academy of Science 234:94-100.

Dott, A.B. and R.K. McKelvy. 1977. "Influence of Ethyl Alcohol in Moderate Levels on Visual Stimulus Tracking." Human Factors 19(2): 191-199.

Drew, G.C., H.P. Colquhown, and H.A. Long. 1958. "Effect of Small Doses of Alcohol on a Skill Resembling Driving." British Medical Journal 2:993-999.

Duker, H. 1956. "Uber die Wirkung sehr Geringer Alkoholmengen auf die Geistege Leistungsfahigkeit." Arch. Exp. Path. Pharmak. 228:175-176.

Dureman, I. and B. Norman. 1975. "Clinical and Experimental Comparison of Diazepam, Chlorazepate and Placebo." Psychopharmacologia $40: ? 79-284$.

Eggleton, M.G. 1941. "The Effect of Alcohol on the Central Nervous System." British Journal of Psychology 32:52-61.

Eich, J.E. 1977. "State-dependent Retrieval of Information in Human Episodic Memory." In Alcohol and Human Memory, eds. I.M. Birnbaum and E.S. Parker, pp. 14T-757. ErTbaum, HilTsdale, New Jersey.

Elsass, P., et al. 1980. "Kinetics and Neuropsychologic Effects of IV Diazepam in the Presence and Absence of its Active $\mathrm{N}$-Desmethy Metabolite in Humans." Psychopharmacology 70:307-312.

Enzer, N., E. Simonson, and G. Ballard. 1944. "The Effect of Small Doses of Alcohol on the Central Nervous System." American Journal of Clinical Pathology $14: 333-341$.

Epstein, L.C. and L. Lasagna. 1968. "A Comparison of the Effects of Orally Administered Barbiturate Salts and Barbiturate Acids on Human Psychomotor Performance." The Journal of Pharmacology and Experimental Therapeutics, T64(2):433-441.

Erwin, C.W., et al. 1978. "Alcohol-Induced Drowsiness and Vigilance Performance." Journal of Studies on Alcohol 39:505-516.

Evans, H. and K. Davis. 1969. "Dose Response Effects of Secobarbital on Human Memory." Psychopharmacologia 14:46-61.

Fabian, M., 0.A. Parsons, and J.A. Silberstein. 1981. "Impaired Perceptual-Cognitive Functioning in Women Alcoholics: Cross-Validated Findings." Journal of Studies on Alcohol 42:217-229.

Fang, J.S. 1982. "Cocaine Spreads Its Deadly Net." U.S. News and Wor Id Report 92:27-79.

Fink, M. 1971. Summary Report: Marihuana Study Program 278M7C. Prepared for the National Institute of Mental Health. 
Fischman, M.W. and C.R. Schuster. 1980. "Cocaine Effects in Sleep-Deprived Humans." Psychopharmacology 72:1-8.

Fischman, M.W., et al. 1976. "Cardiovascular and Subjective Effects of Intravenous Cocaine Administration in Humans." Archives of General Psychiatry 33(2):983-989.

Fishburne, P.M., H.I. Abelson, and I.H. Cisin. 1980. National Survey on Drug Abuse: Main Findings 1979. Superintendent of Documents, Washington, D.C.

Fisher, G. and H.R. Brickman. 1973. "Multiple Drug Use of Marihuana Users." Diseases of the Nervous System 34:40-43.

Fitts, P.M. and M.I. Posner. 1967. Human Performance. Brooks/Cole Publishing Company, Belmont, California.

Forbes, G. 1947. "The Effect of Alcohol on the Psychomotor Reactions as a Possible Index of the Degree of Alcohol Intoxication." Medico-Legal Journal 15:23-38.

Forrest, G., T. Bortner, and C. Bakker. 1967. "The Role of Personality Variables in Response to Chlorpromazine, Dextroamphetamine and Placebo." Journal of Psychiatric Research 5:281-288.

Frankenhaeuser, M., A.L. Myrsten, and G. Jarpe. 1962. "Effects of a Moderate Dose of Alcohol on Intel lectual Functions." Psychopharmacologia 3:344-351.

Fraser, J.0. 1949. "Withdrawal Symptoms in Cannabis Indica Addicts." Lancet $2: 747-748$.

Freudenberger, H.J. and G. Richelson. 1980. Burn-Out: The High cost of Hjgh Achievement. Anchor Press: Doubleday and Company, Garden City, New York.

Frey, D. 1983. "What! Alcoholism in the Fire Service?" Speaking of Fire, Spring.

Frowein, H.W. 1981. "Selective Effects of Barbiturate and Amphetamine on Information Processing and Response Execution." Acta Psychologica 47(2): 105-115.

Frowein, H.W. and A.F. Sanders. 1978. "Effects of Amphetamine and Barbiturate in a Serial Reaction Task Under Paced and Self-Paced Conditions." Acta Psychologica 42:263-276.

Ganowsky, D.S., et al. 1979. "Marijuana and Interpersonal Interactions." Archives of General Psychiatry 35:512-527.

Gaston, M. and N.R. Walker. 1981. "Psychopharmacology." In Preparatory Course for the National and State Licensing Examinations in psychology. Association for Advanced Training in the Behavioral Sciences, Berkeley, California. 
Ghoneim, M. and S. Mewaldt. 1975. "Effects of Diazepam and Scopolamine on Storage, Retrieval and Organizational Processes in Memory." Psychopharmacologia $44: 257-262$.

Ghoneim, M. and S. Mewaldt. 1977. "Studies on Human Memory: the Interaction of Diazepam, Scopolamine and Physostigmine." Psychopharmacology 52:1-6.

Ghoneim, M., S. Mewaldt, and J. Thatcher. 1975. "The Effect of Diazepam and Fentanyl on Mental, Psychonotor and Electroencephalographic Functions and Their Rate of Recovery." Psychopharmacologia 44:61-65.

Ghoneim, M., et a). 1981. "Memory and Performance Effects of Single and 3-Week Administration of Diazepam." Psychopharmacology $73: 147-151$.

Goldberg, L. 1943. "Quantitative Studies on Alcohol Tolerance in Man. The Influence of Ethyl Alcohol on Sensory, Motor and Psychological Functions Referred to Blood Alcohol in Normal and Habituated Individuals." Acta Physiol. Scand. 5:1-128.

Goldenberg, I. 1972. Employment and Addiction: Perspectives on Existing Bus iness and Treatment Practices. Harvard University, Graduate School of Education, Cambridge, Massachusetts.

Goldstein, A., B. Searle, and R. Schimke. 1960. "Effects of Secobarbital and of d-Amphetamine on Psychomotor Performance of Normal Subjects." Journal of Pharmacological and Experimental Therapeutics 130:55-58.

Goldstone, S., W.T. Lhamon, and H.G. Nurnberg. 1978, "Effect of Alcohol on Temporal Information Processing." Perceptual and Motor Skills $46: 1310$.

Goodwin, D.W., B. Powell, and J. Stein. 1973. "Behavioral Tolerance to Alcohol in Moderate Drinkers." American Journal of Psychiatry $122: 93-94$.

Greenblatt, D.J. and R.I. Shader. 1978. "Dependence, Tolerance, and Addiction to Benzodiazepines: Clinical and Pharmacokinetic Considerations." Drug Metabolism Reviews 8:13-28.

Griffiths, R., et al. 1977. "Drug Produced Changes in Human Social Behavior: Facilitation by d-Amphetamine." Pharmacology and Biochemistry of Behavior 7:365-372.

Haffner, J., et al. 1973. "Mental and Psychomotor Effects of Diazepam and Ethano1." Acta Pharmacologica et Toxicologica 32:161-178.

Halpern, J., et al. 1977. The Relationship of Alcohol Treatment to Client Earnings. Center for Social Research and DeveTopment, Denver, Colorado. 
Hart, J., et a1. 1976. "The Effects of Low Doses of Amylobarbitone Sodium and Diazepam on Human Performance." British Journal of Clinical Pharmacology 3:289-298.

Hart ley, L., J. Couper-Smartt, and T. Henry. 1977. "Behavioral Antagonism Between Chlorpromazine and Noise in Man." Psychopharmacology $55: 97-102$.

Hartocollis, P. and D. Johnson. 1956. "Differential Effects of Alcohol on Verbal Fluency." Quarterly Journal of Studies on Alcohol $17: 183-189$.

Hauty, G.T. and R.B. Payne. 1958. "Effects of Analeptic and Depressant Drugs on Psychological Behavior." American Journal of Public Health 48(5):571-577.

Healy, T., J. Robinson, and M. Vickers. 1970. "Physiological Responses to Intravenous Diazepam as a Sedative for Conservat ive Dentistry." British Medical Journal 3:10-13.

Hendler, N., et a1. 1980. "A Comparison of Cognitive Impairment Due to Benzodiazepines and to Narcotics." American Journal of Psychiatry $137: 828-830$.

Hillestad, L., T. Hansen, and H. Melsom, 1974. "Diazepam Metabolism in Normal Man. II. Serum Concentration and Clinical Effect After Oral Administration and Cumulation." Clinical Pharmacology and Therapeutics 16:485-489.

Hindmarch, I. and A. Gudgeon. 1980. "The Effects of Clobazam and Lorazepam on Aspects of Psychomotor Performance and Car Handling Ability." British Journal of Clinical Pharmacology 10:145-150.

Hodges, C.J., Jr. 1976. "Operator Tasks." In Conference Proceedings: Work shop on Power Plant Operator Selection Methods, ed, R.W. Pack. Report No. EPRI-SR-28. Electric Power Research Inst itute.

Holzman, P., D. Levy, E. Uh lenhuth, L. Proctor, and D. Freedman. 1975. "Smooth Pursuit Eye Movements and Diazepam, CPZ, and Secobarbita1." Psychopharmacologia $44: 111$.

Honneger, H., R. Kampschulte, and H. Klein. 1970. "Alcohol Disturbance of Visual Acuity for Moving Objects." Blutalkohol 7:31-44.

Howells, D.E. 1956. "Nystagmus as a Physical Sign in Alcoholic Intoxication." British Medical Journal 1:1405-1406.

Huntley, M.S. 1974. "Effects of Alcohol, Uncertainty, and Novelty Upon Response Selection." Psychopharmacologia 39:259-266. 
Huntley, M.S. and M.W. Perrine. 1971. "Influence of Alcohol on Driving Behavior in an Instrumented Car." Paper presented at the Symposium on Psychological Aspects of Driver Behavior, Hoordwijkerhoust, The Nether lands.

Hurst, P., R. Radlow, and M. Weidner. 1968. "Effects of d-Amphetamine on Task-Alternation and Utility of Delayed Reward." American Journa] of Psychology 81:391-397.

Hutt, S., P. Jackson, A. Belsham, and G. Higgins. 1968. "Perceptual-motor Behavior in Relation to Blood Phenobarbitone Level." Developmental Medicine and Child Neurology 10:626-632.

Institute of Medicine. 1979. Sleeping Pills, Insomnia, and Medical Practice. National Academy of Sciences, Washington, D.C.

Institute of Nuclear Power Operations. 1982. 1982 Survey of NuclearRelated Occupational Employment in United States Electric Utifities. AtTanta, Georgia.

Jaattela, A., et a1. 1971. "The Effects of Diazepam or Diphenhydramine on Healthy Human Subjects." Psychopharmacologia 21:202-211.

Janowsky, D., et al. 1979. "Marijuana and Interpersonal Interactions." Archives of General Psychiatry 133:384-388.

Jellinek, E.M. 1960. The Disease Concept of Alcoholism. Hillhouse, New Brunswick, New Jersey.

Jellinek, E.M. and R.A. McFarland. 1940. "Analys is of Psychological Experiments on the Effects of Alcohol." Quarterly Journal of Studies on Alcohol $1: 272-371$.

Jennings, J., C. Wood, and B. Lawrence. 1976. "Effects of Graded Ooses of Alcohol on Speed-accuracy Tradeoff in Choice Reaction Time." Perception and Psychophysics 19:85-91.

Jones, B.M. and A. Vega. 1972. "Cognitive Performance Measured on the Ascending and Descending Limb of the Blood Alcohol Curve." Psychopharmacologia 23:99-114.

Jones, B.M. and M.K. Jones. 1977. "Alcohol and Memory Impairment in Male and Female Social Drinkers." In Alcohol and Human Memory, eds. I.M. Birnbaum and E.S. Parker, pp. 128-T38. ErTbaum, HiTTsdale, New Jersey.

Jones, D., M. Lewis, and T. Spriggs, 1978. "The Effects of Low Doses of Diazepam on Human Performance in Group Administered Tasks." British Journal of Clinical Pharmacology $6: 333-337$. 
Jones, E.R. and A.J. Eschenbrenner. 1982. "Simulators as Selection Devices for Nuclear Power Plant operators." In The Human Equation in Electric Power Plants: A Symposium, ed. P.D. DuBois. Center for Nuclear Studies, Memphis State University, Memphis, Tennessee.

Jones, R.K. and K.B. Joscelyn. 1978. Alcohol and Highway Safety 1978: A Review of the State of knowledge. D0T-HS-5-0.T2T7. U.S. Department of Transportation, National Highway Traffic Safety Administration, Washington, pp. 35-49.

Julien, R.M. 1981. A Primer of Drug Action. San Francisco: Freeman and Company.

Kagel, J.H., R.C. Battalio, and C.G. Miles. 1980. "Marihuana and Work Performance: Results from an Experiment." Journal of Human Resources 15(3):373-395.

Klein, R.H. and H.R. Jex. 1975. "Effects of Alcohol on a Critical Tracking Task." Journal of Studies on Alcohol 36:11-20.

Kleinknecht, R. and D. Donaldson. 1975. "A Review of the Effects of Diazepam on Cognitive and Psychomotor Performance." Journal of Nervous and Mental Disease 161:399-411.

Klerman, G.L., et a1. 1960. "Sedation and Tranquilization: A Comparison of the Effects of a Number of Psychopharmacologic Agents upon Normal Human Subjects." Archives of Genera] Psychiatry 3:4-13.

Kolata, G. 1982. "New Valiums and Anti-Valiums on the Horizon." Science $216: 604-605$.

Kornetsky, C., et al. 1959. Journal of Pharmacological Experimental Therapy $127: 46$.

Kupietz, S.S., et al. 1980. "Effects of Methylphenidate on Learning a 'Beginning Reading Vocabulary' by Normal Adults." Psychopharmacology $69: 69-72$.

Labor Department Employment and Training Administration. 1977. Dictionary of Occupational Tities. 4th Edition. U.S. Government printing office, Washington, D.C.

La1, H., et al. 1972. "Failure to Demonstrate 'Amphetamine State' Controlling Learned Behavior in Humans." Clinical Toxicology 5:43.

Lambo, T.A. 1965. "Medical and Social Problems of Drug Addiction in West Africa, with Special Emphasis on Psychiatric Aspects." United Nations Bullet in on Narcotics 17:3-13.

Landauer, A., D. Pocock, and F. Prott. 1974. "The Effect of Medazepam and Alcohol on Cognitive and Motor Skills Used in Car Driving." Psychopharmacologia 37: 159-168. 
Laties, V. and B. Weiss. 1966. "Performance Enhancement by the Amphetamines: a New Appraisal." In Proceedings of the 5th International Congress of the College of International

Neuropsychopharmacology, pp. 800. Excerpta Medica, Amsterdam, The Netherlands.

Laure11, H. 1977. "Effects of Small Doses of Alcohol on Driver Performance in Emergency Traffic Situations." Accident Analys is and Prevention 9:191-201.

Levain, G., et al. 1972. Cannabis: a Report of the Commission of Inquiry into the Non-Medical Use of Drugs. Information Canada, ottawa, pp. 143-144.

Lehembre, J. 1963. "An Experimental Study of the Effect of Psychotropic Drugs on Mental Function." Neder 1. T. Geneesk 107:1227.

Levens, E. 1976. "The Cost-Benef it and Cost-Effectiveness of Occupationa 1 Alcoholism Programs." Professional Safety.

Levine, J.M., G.D. Greenbaum, and E.R. Notkin. 1973. The Effect of Alcohol on Human Performance: a Classification and Integration of Research Findings. American Institute for Research, Washington, D.C.

Levy, O.L., R.B. Lipton, and P.S. Holzman. 1981. "Smooth Pursuit Eye Movements: Effects of Alcohol and Chloral Hydrate." Journal of Psychiatric Research 16:1-11.

Lewis, E.G., R.E. Oustman, and E.C. Beck. 1969. "The Effect of Alcohol on Sensory Phenomena and Cognitive and Motor Tasks." Quarterly Journal of Studies on Alcohol 30:618-633.

Lewis, E.M., Jr. 1972. Interaction of Age and Alcohol on Dark Adaptation Time. ICRL-RR-68-4. U.S. Department of HeaTth, Education and WeTfare, Washington, D.C.

Lewis, E.M., Jr. and K. Sarlanis. 1969. The Effects of Alcohol on Decision Making with Respect to Traffic SignaTs. ICRL-RR-68-4. U.S. Department of Health, Education and Welfare, Washington, D.C.

Light, W. and C. Keiper. 1971. Effects of Moderate Blood Alcohol Levels on Automotive Passing Behavior. ICRL-RR-69-4. U.5. Public Health Service, Injury Control Research Laboratory, Providence, Rhode Is land.

Liljequist, R., M. Linnoila, and M.S. Mattila. 1974. "Effects of Two Weeks' Treatment with Chlorimipramine and Nortriptyline, Alone or in Combination with Alcohol, on Learning and Memory." Psychopharmacologia 39:181-186.

Liljequist, R., M. Linnoila, and M. Mattila. 1978. "Effect of Diazepam and Chlorpromazine on Memory Functions in Man." European Journal of Clinical Pharmacology 13:339-343. 
Liljequist, R., M. Mattila, and M. Linnoila. 1981. "Alterations in Human Memory Following Acute Malprotiline, Diazepam and Codeine Administration." Acta Pharmacologica et Toxicologica 48:190-192.

Li1jequist, R., et al. 1975. "Effect of Two Weeks' Treatment with Thioridazine, Ch Jorpromazine, Sulpiride, and Bromazepam, Alone or in Combination with Alcohol, on Learning and Memory in Man." Psychopharmacologia $44: 205-208$.

Linnoila, M., et a1. 1978. "Effects of Alcohol on Psychonotor Performance of Men and Women." Journal of Studies on Alcohol $39: 745-758$.

Linnoila, M. 1976. "Tranquilizers and Driving." Accident Analysis and Prevention 8(1):15-19.

Linnoila, M. and S. Hakkinen. 1974. "Effects of Diazepam and Codeine, Alone and in Combination with Alcohol, on Simulated Driving." Clinical Pharmacology and Therapeutics 15:368-373.

Linnoila, M. and M. Maki. 1974. "Acute Effects of A'lcohol, Diazepam, Thioridazine, Flupenthixole, and Atropine on Psychomotor Performance Profiles." Arzneim. Forsch. 24:565-569.

Linnoila, M. and M.J. Mattila. 1973. "Drug Interaction on Psychomotor Skills Related to Driving: Diazepam and Alcohol." European Journal of Clinical Pharmacology $5: 186-194$.

Linnoila, M. and M.J. Mattila. 1973. "Interaction of Alcohol and Drugs on Psychomotor Skills as Demonstrated by a Driving Simulator." British Journal of Pharmacology 47:67 IP-672P.

Longhetti, A. and L. Barnett. 1965. "Report of a County-wide Educational Program Regarding the Drinking Driving Problem." Proceedings of the 4 th International Conference on Alcohol and Traffic Safety, Bloomington, Indiana.

Loomis, T.A. and T.C. West. 1958. "The Influence of Alcohol on Automobile Driving Ability." Quarterly Journal of Studies on Alcohol 19:30-46.

Lovibond, S.H. and K. Bird. 1972. "Effects of Blood Alcohol Level on the Driving Behavior of Competition and Non-competition Drivers." Paper presented at the 29 th International Congress on Alcoholism and Drug Dependence, Australia.

Lubin, R.A. 1979. "Influences of Alcohol, Interpersonal Feedback, and Drinking Experience Upon Performance and Judgment." Perceptual and Motor Sk ills 48:95-104.

Lubin, R.A. 1977. "Influences of Alcohol Upon Performance and Performance Awareness." Perceptual and Motor Skills 45:303-310. 
Lusterman, S. 1974. Industry Roles in Health Care. Conference Board, New York.

Macavoy, M.G. and D.F. Marks. 1975. "Divided Attention Performance of Cannabis Users and Non-Users Following Cannabis and Alcohol." Psychopharmacologia $44: 147-152$.

MacLeod, S., et al. 1977. "Diazepam Actions and Plasma Concentrations Following Ethanol Ingestion." European Journal of Clinical Pharmacology 11:345-349.

Maling, H.M. 1970. "Toxicology of Single Doses of Ethyl Alcohol." In International Encyclopedia of Pharmacology and Therapeutics, Vol. 2, Section 20, "Alcohols and Derivatives." Pergamon Press, New York.

Malpas, A. and C. Joyce. 1969. "Effects of Nitrazepam, Amylobarbitone and Placebo on Some Perceptual, Motor and Cognitive Tasks in Normal Subjects." Psychopharmacologia 14:167-177.

Mannello, T.A., et a1. 1979. Prevalence, Costs, and Handling of Drinking Problems on Seven Railroads. University Research Corporation, Washington, D.C.

Manno, J., et al. 1971. "The Influence of Alcohol and Marihuana on Motor and Mental Performance." Clinical Pharmacology and Therapeutics $12: 202-211$.

Marcovitz, E, and H.J. Myers. 1944. "The Marihuana Addict in the Army." War Medicine 6:382-391.

Marden, P.G. 1980. A Procedure for Est imating the Potential Clientele of Alcoholism Service Programs. U.5. Department of Health, Education, and Welfare Publication No. (ADM) 80-908. National Institute on Alcohol Abuse and Alcoholism, Washington, D.C.

McDonald, A. 1972. "The Effects of d-Amphetamine on the Intelligence Test Scores of Normal Human Subjects." Proceedings of the 80th Annual Convention of the American Psychological Association, pp. 809-810.

McKay, A. and J. Dundee. 1980. "Effect of Oral Benzodiazepines on Memory." British Journal of Anaesthesiology 52:1247-1257.

McNair, D. 1973. "Antianxiety Drugs and Human Performance." Archives of General Psychiatry 29:611-617.

Mendhiratta, S.S., N.N. Wig, and S.K. Verma. 1978. "Some Psychological Correlates of Long-term Heavy Cannabis Users." British Journal of Psychiatry 132:482-486. 
Miles, C.G., et al. 1975. "The Effects of Cannabis and Negotiated Wage Rate Changes on Income and Job Performance in an Experimental Token Economy." In Experimentation in Controlled Environment, ed. C.G. Miles, pp. 57-69. Addiction Research Foundation, Toronto.

Miller, L.L., et al. 1977a. "Marijuana: Effects on Free Recall and Subjective Organization of Pictures and Words." Psychopharmacology $55: 257-262$.

Miller, L.L., et al. 1977b. "Marijuana: Effects on Storage and Retrieval of Prose Material." Psychopharmacology 51:311-316.

Miller, R.D., et al. 1972. "A Comparison of De1ta-9-Tetrahydrocannabinol and Marijuana Effects in Humans." In Cannabis: a Report of the Commission of Inquiry into the Non-Medical Use of Drugs, ed. G.E. Lebain, pp. T3T-136. Information Canada, ot tawa.

Milner, G. and A.A. Landauer, 1971. "Alcohol, Thioridazine and Chlorpromazine Effects on Skills Related to Driving Behavior." British Journal of Psychiatry 118:351-352.

Milner, G. and A. Landauer. 1973. "Haloperidol and Diazepam Alone and Together with Alcohol, in Relation to Driving Safety." Blut Alkohol 10:247-254.

Milstein, S.L. et al. 1975. "Marijuana-Produced Impairments in Coordination." The Journal of Nervous and Mental Disease $161(1): 26-31$.

Milt, H. 1974. Basic Handbook on Alcoholism. Scientific Aids Publications, New Jersey.

Mirsky, A.F. and C. Kornetsky. 1964. "On the Dissimilar Effects of Drugs on the Digit Symbol Substitution and Continuous Performance Tests." Psychopharmacologia 5:161-177.

Mohs, R.C., et a1. 1978. "Methamphetamine and Diphenhydramine Effects on the Rate of Cognitive Processing." Psychopharmacology 49:275-279.

Mohs, R.C., et aI. 1980. "Sensitivity of Some Human Cognitive Functions to Effects of Methamphetamine and Secobarbital." Drug and Alcohol Dependence 5:145-150.

Morland, J. et a1. 1974. "Combined Effects of Diazepam and Ethanol on Mental and Psychomotor Functions." Acta Pharmacologica et Toxicologica $34: 5-15$.

Mortimer, R.G. 1963. "Effect of Low Blood Alcohol Concentrations in Simulated Day and Night Driving." Perceptual and Motor Skills $17: 399-408$. 
Moskowitz, H. 1974. "Alcohol Influences Upon Sensory Motor Function, Visual Perception, and Attention." In Alcohol, Drugs, and Driving, ed. M.W. Perrine. DOT-HS-801-096. National Highway Traffic Safety Administration, Washington, D.C.

Moskowitz, H. and M. Burns. 1971. "Effect of Alcohol on the Psychological Refractory Period." Quarter iy Journal of Studies on Alcohol 32:782-790.

Moskowitz, H. and D. DePry. 1968. "Differential Effect of Alcohol on Auditory Vigilance and Divided-attention Tasks." Quarterly Journal of Studies on Alcohol 29:54-63.

Moskowitz, H. and W. McGlothlin. 1974. "Effects of Marihuana on Auditory Signal Dectection." Psychopharmacologia 40:137-145.

Moskowitz, H. and S. Roth. 1971. "Effect of Alcohol on Response Latency in Object Naming." Quarterly Journal of Studies on Alcoho? 32:969-975.

Moskowitz, H., S. Sharma, and W. McGlothlin. 1972. "The Effect of Marihuana Upon Peripheral Vision as a Function of Information Processing Demands on Central Vision." Perceptual and Motor Skills $35: 875-882$.

Moskowitz, N. 1973. "Laboratory Studies of the Effects of Alcohol on Some Variables Related to Driving." Journal of Safety Research 5: 185-199.

Mule, S.J. 1976. Cocaine: Chemical, Biological, clinical, Social, and Treatment Aspects. Cleveland, ohio: CRC Press.

Murdock, B.B. 1974. Human Memory: Theory and Data. Wiley, New York.

National Commission on Marihuana and Drug Abuse. 1972. Marihuana: a Signal of Misunderstanding. Technical Papers of the First Report of the NationaT Commission on Marihuana and Drug Abuse. U.S. Government Printing office, Washington, D.C.

National Institute of Alcohol Abuse and Alcoholism. 1978, 1981. 3rd Special Report to the United States Congress on Alcohol and HeaTth. 4th Special Report to the United States Congress on ATcohol and Health. United States Department of Health and Human Services, Washington, D.C.

National Observer, December 11, 1976, p. 14B.

Newman, H. and E. Fletcher. 1941. "The Effect of Alcohol on Vision." American Journal of the Medical Sciences 202:723-731.

Newman, H.W. 1947. "Variability in Tolerance to Depressant Drugs." Stanford Medical Bulletin 5:12-14. 
Newman, H.W. and M. Abramson. 1941. "Relation of Alcohol Concentration to Intoxication." Proceedings of the Society of Experimental Biology 48:509-572.

NUREG/CR-1750. 1981. "Analys is, Conclusions, and Recommendations Concerning Operator Licensing." National Technical Information Service, Springfield, Virginia.

NUREG-0903. 1982. "Survey of Industry and Government Programs to Combat Drug and Alcohol Abuse." National Technical Information Service, Springfield, Virginia.

Noblit, G.W., et al. 1975. Programatic Responses to Drug Abuse in Public Sector Employment: a Research Report. International Personnel Management Association, Washington, D.C.

O'Hanlon, J., et al. 1982. "Diazepam Impairs Lateral Position Control in Highway Driving." Science 217:79-81.

Orr, J., et a1. 1976. "Relation Between Drug-Induced Central Nervous System Effects and Plasma Levels of Diazepam in Man." Modern Problems in Pharmacopsychiatry 11:57-67.

Oswald, I., et al. 1979. "The Effects of Two Hypnotics on Sleep, Subjective Feelings and Skilled Performance." Advances in the Bio-Sciences 21:51-65.

Parker, E.S., et al. 1981. "The Alcohol Facilitation Effect on Memory: a Dose-Response Study." Psychopharmacology 74:88-92.

Parker, E.S., et al. 1974. "Alcohol and the Disruption of Cognitive Processes." Archives of General Psychiatry 31:824-828.

Parker, E.S., et al. 1980. "Retrograde Enhancement of Human Memory with Alcohol." Psychopharmacology 69:219-222.

Parker, E.S. and E.P. Noble. 1977. "Alcohol Consumption and Cognitive Functioning in Social Drinkers." Journal of Studjes on Alcohol 38: 1224- 1232 .

Parry, H.J., et al. 1973. "National Patterns of Psychotherapeutic Drug Use." Archives of General Psychiatry 28:769-784.

Payne, R. and G. Hauty. 1954. Journal of Experimental Psychology $47: 267$.

Payne, R. and G. Hauty. 1955. Journal of Aviation Medicine 25:382.

Pearl, J.H., E.F. Domino, and P. Rennick. 1973. "Short-Term Effects of Marijuana Smoking on Cognitive Behavior in Experienced Male Users." Psychopharmacologia 31:13-24. 
Pearson, R.G. and G.L. Neal. 1970. "Operator Performance as a Function of Drug, Hypoxia, Individual, and Task Factors." Aerospace Medicine 4 r: $154-158$.

Peeke, S.C., R.T. Jones, and G.C. Stone. 1976. "Effects of Practice on Marijuana-Induced Changes in Reaction Time." Psychopharmacology 48: 159-163.

Perrine, M.W. 1974. Alcohol Experiments on Driving-Related Behavior: a Review of the 1972-1973 Literature--Alcohol Countermeasures Literature Review. DOT-HS-80T-266. U.S. Department of Transportation, National Highway Traffic Safety Administration, Washington, D.C.

Peters, B.A., et a]. 1976. "Sensory, Perceptual, Motor and Cognitive Functioning and Subjective Reports Following Oral Administration of Delta-9-Tetrahydrocannab ino 1." Psychopharmacology 47(2): 14 1-148.

Petersen, R. and M. Ghoneim. 1980. "Diazepam and Human Memory: Influence on Acquisition, Retrieval, and State-dependent Learning." Progress in Neuro-Psychopharmacology 4:81-89.

Peterson, R.C. and R.C. Stillman. 1977. Cocaine: 1977. NIDA Research Monograph \#13. Washington, D.C.: U.S. Government Printing Office.

Physicians' Desk Reference. 1982. Medical Economics Company, Oradel1, New Jersey.

Poh1, J.E.F. 1978. "Blood Alcohol and Impairment of Judgment." Clinjcal Science and Molecular Medicine 55:57-61.

Post, R.M., J. Kotin, and F.K. Goodwin. 1974. "The Effects of Cocaine on Depressed Patients." American Journal of Psychiatry, $131(5): 511-517$.

Poulos, C.X., et al. 1981. "Acquisition of Tolerance to AlcoholInduced Memory Deficits in Humans." Psychopharmacology 73:176-179.

Pradhan, S.N. and S.N. Dutta, eds. 1977 Drug Abuse: Clinical and Basic Aspects. C.V. Mosby Co., St. Louis.

Prag, J.J. 1953. "The Chemical Diagnosis of Alcoholic Intoxication." South African Journal of Medical Science 18:141-154.

Price, D.L. and R.A. Flax. 1982. "Alcohol Task Difficulty and Incentives in Drill Press Operation." Human Factors 24:573-579.

Price, D.L. and T.G. Hicks. 1979. "The Effects of Alcohol on Performance of a Production Assembly Task." Ergonomics 22:37-41. 
Price, D.L, and R. Liddle. 1982. "The Effects of Alcohol on Performance of a Welding Task." Welding Journal 61:15-19.

Rabin, A. and H. Blair. 1953. "The Effects of Alcohol on Handwriting." Journal of Clinical Psychology 9:284-287.

Reid, L., R. Hansteen, and R. Miller. 1972. "The Effects of Cannabis and Alcohol on Psychomotor Tracking Performance." In Cannabis: A Report of the Commission of Inquiry into the Non-Medical Use of Orugs, ed., G. Dedain, pp. 7T-83. Information Canada, Ottawa.

Reinish, H. 1971. Identifying On-the-Job Behavioral Manifestations of Drug Abuse: a Guide for Work Supervisors. Mobilization for Youth, Inc., New York.

Reynolds, E. and R. Travers. 1974. "Serum Anticonvulsant Concentrations in Epileptic Patients with Mental Symptoms." British Journal of Psychiatry $124: 440-445$.

Rickles, W.H., Jr., et a1. 1973. "Marjjuana Induced State-Dependent Verbal Learning." Psychopharmacologia 30:349-354.

Ritchie, J.M. and P.J. Cohen. 1975. "Local Anesthetics." In The Pharmacological Basis of Therapeutics, eds. L.S. Goodman and A. Gilman, pp. 379-403. New York: Macmillan.

Roth, T., et a1. 1980. "The Effects of Flurazepam, Lorazepam, and Triazolam on Sleep and Memory." Psychopharmacology 70:231-237.

Roth, W.T., et al. 1973. "The Effect of Marihuana on Tracking Task Performance." Psychopharmacologia 33:259-265.

Rubin, V. and L. Comitas. 1972. A Study of the Effects of Chronic Ganja Smoking in Jamajca. Prepared for the National Institute of Mental Health at the Research Institute for the Study of Man, New York.

Runde11, 0.H., H.L. Williams, and B.K. Lester. 1978. "Comparative Effects of Alcohol, Secobarbital, Methaqualone, and Meprobamate on Information Processing and Memory." In Advances in Experimenta1 Medicine and 8iology, ed. M.M. Gross, pp. 617-628.

Runde11, 0.H., H.L. Willians, and B.K. Lester. 1978. "Secobarbital and Information Processing." Perceptual and Motor Skills 46:1255- 1264 .

Runde11, 0.H. and H.L. Williams. 1979. "Alcohol and Speed-Accuracy Tradeoff." Human Factors 2 1:433-443.

Runde 11, 0.H. and H.L. Williams. 1977. "Effects of Alcohol on Organizational Aspects of Human Memory." In Currents in Alcoholism: Psychiatric, Psychological, Socfal and Epidemjological Studies, ed. F.A. Sejxas, pp. 175-186. Grune and Stratton, New York. 
Ryback, R.S. 1977. "The Intoxicated Goldfish as a Model for Alcohol Effects on Memory in Humans." In Alcohol and Human Memory, eds. I.M. Birnbaum and E.S. Parker, pp. 109-T26. Erlbaum, Hilisdale, New Jersey.

Ryback, R.S. and P.J. Dowd. 1970. "Aftereffects of Various Alcoholic Beverages on Positional Nystagmus and Coriolis Acceleration." Aerospace Medicine 41:429-435.

Salvendy, G. and G.P. McCabe, Jr. 1975. "Marijuana and Human Performance." Human Factors 17(3):229-235.

Schaefer, C.F., C.G. Gunn, and K.M. Dubowski. 1977. "Dose-Related Heart-Rate, Perceptual, and Decisional Changes in Man Following Marihuana Smoking." Perceptual and Motor Skills 44:3-16.

Scher, J.M. 1973. "The Impact of the Drug Abuser on the Work Organization." In Drug Abuse in Industry, ed. J.M. Scher. Charles C. Thomas, Springfield, ITinois.

Schweitzer, H. 1955. "Graphologische, Klinioche und Psychologische Untersuchungen unter Alkoholwirkwng." 0ff. GesundhDienst. 16:361-372.

Seashore, R. and A. Ivy. 1953. Psychological Monographs 67:\#15.

Segalla, E. 1982. "Employee Assistance Programs for Local Governments." Management Information Service Report 14(8):1-14.

Selye, H. 1956. The Stress of Life. McGraw-Hill, New York.

Seppala, T., et al. 1976. "Residual Effects and Skills Related to Driving After a Single Oral Administration of Diazepam, Medazepam or Lorazepam." British Journal of Clinical Pharmacology 3:831-841.

Sexias, F.A., et a]. 1976. "A Definition of Alcoholism." Annals of Internal Medicine 5:764.

Shader, R.I. and 0.J. Greenblatt. 1977. "Clinical Implications of Benzodiazepine Pharmacokinetics." American Journal of Psychiatry $134: 652-655$.

Shain, M. 1982. "Alcohol, Drugs, and Safety: An Updated Perspect ive on Problems and Their Management in the Workplace." Accident Analysis and Prevention 14(3):239-246.

Sharma, S. and H. Mosknwitz. 1974. "Effects of Two Levels of Attention Demand on Vigilance Performance under Marihuana." Perceptual and Motor Skills 38:967-970.

Sharma, S. and H. Moskowitz. 1973. Marijuana Dose Study of Vigilance Performance." Proceedings of the 81st Annual Convention of the American Psychological Association 7:1035-1036. 
Sharma, S. and H. Moskowitz. 1972. "The Effect of Marihuana Upon the Visual Autok inetic Phenomenon." Perceptual and Motor Skills $35: 891-894$.

Siegal, H.A. 1981. "Current Patterns of Psychoactive Drug Use: Some Epidemiologic observations." In Drug and Alcohol Abuse:

Implications for Treatment, ed. S.E. Gardner. Natjonal Institute on Drug Abuse, washington, D.C.

Skegg, D. and J. Perry. 1977. "Use of Medicines in General Practice." British Medical Journal 2:159-165.

Skeg9, D., S. Richards, and R. Doll. 1979. "Minor Tranquillisers and Road Accidents." British Medical Journal 1:917-919.

Sleight, R.B. and K.G. Cook. 1974. Problems in 0ccupational Safety and Health: a Critical Review of Select Worker Physical and psychological Factors. NIOSH 75-124. U.S. Department of Health, Education and Welfare, washington, D.C.

Smith, G. and H. Beecher. 1959. Journal of the American Medical Association 170:542.

Smith, G., M. Weitzner, and H. Beecher. 1963. Journal of Pharmacological Experimental Therapy 139:114.

Smith, T.A. 1982. "A Structural Approach to Setting Up an Employee Assistance Program." Paper presented at the Nineteenth Annual Convention of the American Psychological Association, Washington, D.C.

Spiege1, R. 1979. "Effects of Amphetamines on Performance and on Polygraphic Sleep Parameters in Man." Advances in the Bio-Sciences 21:189-201.

State of Washington, Department of Social and Health Services. 1977. Washington State Alcoholism Plan, Fiscal Year 1978. 0lympia, Washington.

Stening, G. and I. Dureman. 1974. Akuta och Posttariska Effektr av Alkohol pa Prestation i Bilsimulator. Psykologiska Institutionen, Upps la Universitet.

Stephen, M. and R. Prentice. 1978. Developing an Occupational Drug Abuse Program: Considerations and Approaches. National Institute on Drug Abuse, Washington, D.C.

Stitzer, M.L., et al. 1981. "Human Social Conversation. Effects of Ethanol, Secobarbital, and Chlorpromazine." Pharmacology Biochemistry and Behavior 14:353-360. 
Svensson, E., L. Persson, and L. Sjoberg. 1980. "Mood Effects of Diazepam and Caffeine." Psychopharmacology 67:73-80.

Taberner, P.V. 1980. "Sex Differences in the Effects of Low Doses of Ethanol on Human Reaction Time." Psychopharmacology 70:283-286.

Takala, M., E. Siro, and Y. Toivainen. 1958. "Intellectual Functions and Dexterity During Hangover Experiments after Intoxication with $\mathrm{Br}$ andy and with Beer." Quarterly Journal of Studies on Alcohol 19:1-29.

Talland, G.A., J.H. Mendelson, and P. Ryack. 1964. "Experimentally Induced Chronic Intoxication and Withdrawal in Alcoholics. Part 5: Tests of Attention." Quarterly Journal of Studies on Alcohol Supplement No. 2:74 86 .

Tansella, M., C. Zimmermann-Tansella, and M. Lader. 1974. The Residual Effects of N-Desmethyldiazepam in Patients." Psychopharmacologia $38: 81-90$.

Taylor, J. 1982. "The Effects of Benzodiazepines on Cognition and Performance." Unpublished Doctoral Dissertation, University of Washington, Seattle, Washington.

10 CFR 73.56, "Access Authorization Rule."

10 CFR 50.54, "Fitness for Duty Rule."

Tharp, V.K., Jr., et al. 1974. "Alcohol and Information Processing." Psychopharmacologia 40:33-52.

Tharp, V.K., Jr., et al. 1975. "Alcohol and Secobarbital: Effects on Information Processing." Vol. 59. In Alcohol Intoxication and Withdrawal, Experimental Studies, Advances in Experimental Medicine and Biology, ed. M. Gross, pp. 537-552. PTenum, New York.

Thompson, P., F. Huppert, and M. Trimble. 1980. "Anticonvulsant Drugs, Cognitive Function and Memory." Acta Neurologica Scandinavia $80: 75-81$.

Trice, H.M. and P.M. Roman. 1972. Spirits and Demons at Work: Alcohol and Other Drugs on the Job. CorneTT University Press, Ithaca, New York.

Trimble, M.R. and E.H. Reynolds. 1976. "Anticonvulsant Drugs and Mental Symptoms: A Review." Psychological Medicine 6:169-178.

Tuthill, M. 1982. "Joining the War on Drug Abuse." Nation's Business June, pp. 64-65.

"TVA Issues Annual Report." 1980. The Reporter. The News letter of Federal Employee Health and Alcoholism/Drug Abuse Programs. Vol. 11, No. 8, August. 
Urban, M.L. 1973. "Drugs in Industry." In Drug Use in America: Problem in Perspective, $\mathrm{pp} .1136-1152$. U.S. Government Printing office, Washington, D.C.

U.S. Bureau of the Census. 1973. Census of Population: 1970 Subject Reports, Occupational Characteristics. Final Report PC(2)-7A. U.S. Government Printing office, Washington, D.C.

U.S. Nuclear Regulatory Commission. 1982. Information Notice 82-05. Washington, D.C.

Vachon, L., A. Sulkowski, and E. Rich. 1974. "Marihuana Effects on Learning, Attention and Time Estimation." Psychopharmacolgia 39:1-11.

Van Dyke, C. and R. Byck. 1982. "Cocaine." Scientific American 246:128-134t.

Vaness, N. and R. Brown. 1966. "The Effect on Intellectual Performance of a Minimal Dosage of a Depressant (Benadryl-50mg) and of a Stimulant (Dextro-Amphetamine-5mg)." Medical Services Journal of Canada 22:361-367.

Voge1-Sprott, M.D. 1979. "Acute Recovery and Tolerance to Low Doses of Alcohol: Differences in Cognitive and Motor Skill Performance." Psychopharmacology 61:287-291.

Vojtechovsky, M. and V. Safratova. 1971. "The Effects of Some Psychotropic Drugs on Memory Consolidation in Man." Activita Nervosa Superior 13:141-142.

Wallgren, H. and L. Barry. 1970. Actions of Alcohol. Elsevier, Amsterdam, Holland.

Weil, A.T., N.E. Zinberg, and J.M. Nelsen. 1968. "Clinical and Psychological Effects of Marihuana in Man." Science 162:1234-1242.

Weingartner, H. and D.L. Murphy. 1977. "State-dependent Storage and Retrieval of Experience While Intoxicated." In Alcohol and Human Memory, eds. I.M. Birnbaum and E.S. Parker, pp. 159-773. ErTbaum, Hillsdale, New Jersey.

Weiss, B. 1969. "Enhancement of Performance by Amphetamine-like Drugs." In Abuse of Central Stimulants, eds. F. Sjoquist and M. Tottie, pp. 3T-60. Raven Press, New York.

Weiss, B. and V. Laties, 1962. "Enhancement of Human Performance by Caffeine and the Amphetamines." Pharmacology Review 14:1-36.

Weiss, S. 1971. "A General Picture of Charas Habit in Afghanistan." Report from Kabul University Medical School, Kabul, Afghanistan. 
Weitzner, M. 1965. "Manifest Anxiety, Amphetamine, and Performance." Journal of Psychology 60:71-79.

Wittenborn, J. 1979. "Effects of Benzodiazepines on Psychomotor Performance." British Journal of Clinical Pharmocology 7:615-675.

Wittenborn, J., et al. 1976. "The Effect of Minor Tranquilizers on Psychomotor Performance." Psychopharmacology 47:28 $1-286$.

Wittenborn, J., et a1. 1979. "Psychomotor Changes During Initial Day of Benzodiazepine Medication." British Journal of Clinical Pharmacology $7: 69 S-76 S$.

Wolkenberg, R.C., C. Gold, and E.R. Tichauer. 1975. "Delayed Effects of Acute Alcoholic Intoxication on Performance with Reference to Work Safety." Journal of Safety Research 7:104-118.

Woods, J.H. and D.A. Downs. 1973. "The Psychopharmacology of Cocaine." In Drug Use in America: Problem in Perspective. Appendix Volume I: Pat terns and Consequences of Orug Use, pp. TT6-139. U.S. Government Printing office, Washington, D.C.

Wrich, J.T. 1980. The Employee Ass istance Program. Hazeldon, City Center, Minnesota.

Young, J.R. 1970. "Blood Alcohol Concentration and Reaction Time." Quarterly Journal of Studies on Alcohol 31:823-831.

Zirkle, G.A., et al. 1959. "Effects of Chlorpromazine and Alcohol on Coordination and Judgment." Journal of the American Medical Association 171: 1496-1499.

Zirkle, G.A., et al. 1960. "Meprobamate and Small Amounts of Alcohol: Effects on Human Ability, Coordination, and Judgment." Journal of the American Medical Association 173(16):1823-1825.

Zwahlen, H.T. 1976. "The Effect of Alcohol on Driving Skills and Reaction Times." Journal of Occupational Accidents 1:21-38. 
NUREG/CR-3196

PNL -4679

BHARC-400/83/003

RX

\section{DISTRIBUTION}

No. of

Copies

U.S. Nuclear Regulatory Cormission

Division of Technical Information and Document Control

7920 Norfolk Avenue

Bethesda, MD 20014

10 T.G. Ryan

Division of Facility 0perations

Office of Nuclear Regulatory Research

Mail Stop 1130SS

U.S. Nuclear Regulatory Comission

Washington, D.C. 20555

ONSITE

50 Pacific Northwest. Laboratory

V. Barnes

AM Hope

MV McGuire

LR Radford (7)

Wh. Rank in (30)

R. Shikiar

RJ Sorenson

RD Widrig

Publishing Coordination (2)

Technical Information (5) 



\begin{tabular}{|c|c|c|}
\hline \multirow{3}{*}{ 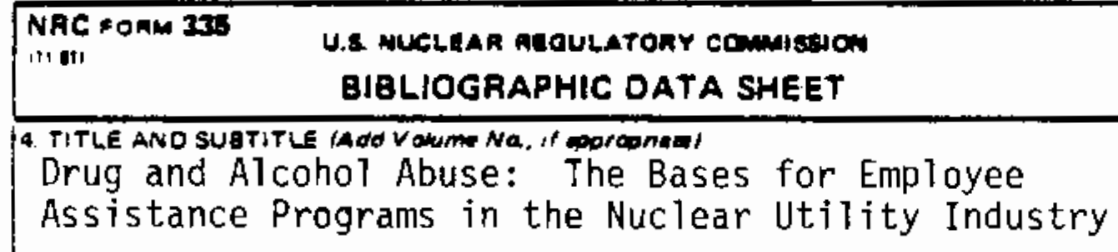 } & \multicolumn{2}{|c|}{ 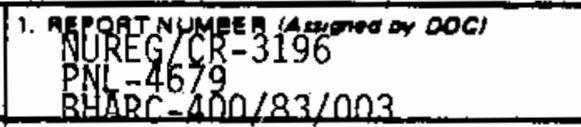 } \\
\hline & \multicolumn{2}{|c|}{ 2. (Lewe oremk) } \\
\hline & \multicolumn{2}{|c|}{ 3. RECIPIENT'S ACCESSION NO. } \\
\hline $\begin{array}{l}\text { 7. AUTHOA(s) L.R. Radford, W.L. Rankin, V. Barnes, } \\
\text { M.V. McGuire, A.M. Hope }\end{array}$ & \multicolumn{2}{|c|}{ 5. DATE REPORT COMPLETEO } \\
\hline \multirow{2}{*}{ 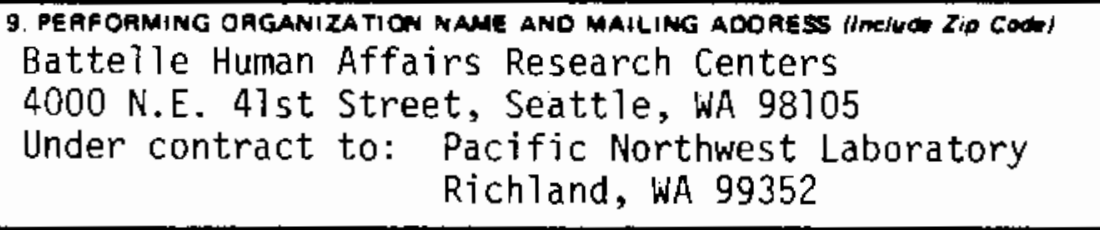 } & \multicolumn{2}{|c|}{\begin{tabular}{|l|l} 
MONTH July & TrEAM 1983 \\
\end{tabular}} \\
\hline & \multicolumn{2}{|l|}{ 9. (Lowe abert) } \\
\hline \multirow{2}{*}{ 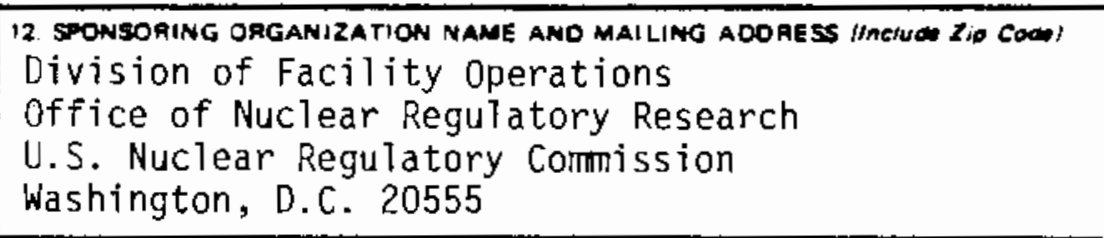 } & \multicolumn{2}{|c|}{ 10. PHOJECT/TASK/MOAX UNIT NO. } \\
\hline & \multicolumn{2}{|l|}{$\begin{array}{r}\text { 11. FN mo. } \\
\text { B2453 }\end{array}$} \\
\hline \multicolumn{3}{|c|}{ 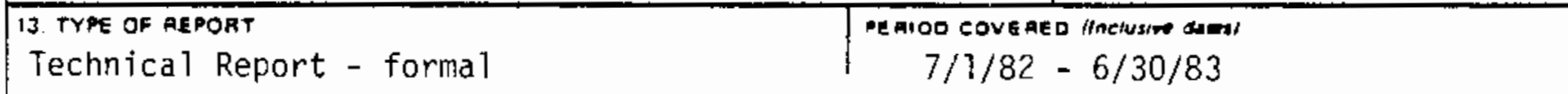 } \\
\hline 15. SLPPLEMEN TARY NOTES & \multicolumn{2}{|l|}{ 14. (Lem ankt } \\
\hline \multicolumn{3}{|c|}{$\begin{array}{l}\text { 16. ABSTRACT } 200 \text { words wast } \\
\text { This report describes the nature, prevalence, and trends of drug and } \\
\text { alcohol abuse among members of the U.S. adult population and among } \\
\text { personnel in non-nuclear industries. Analogous data specific to the } \\
\text { nuclear utility industry are not available, so these data were gathered } \\
\text { in order to provide a basis for regulatory planning. The nature, } \\
\text { prevalence, and trend information was gathered using a computerized } \\
\text { literature search, telephone discussions with experts, and interviews } \\
\text { with employee assistance program representatives from the Seattle area. } \\
\text { This report also evaluates the possible impacts that drugs and alcohol } \\
\text { might have on nuclear-related job performance, based on currently } \\
\text { available nuclear utility job descriptions and on the scientific } \\
\text { literature regarding the impairing effects of drugs and alcohol on human } \\
\text { performance. Employee assistance programs, which can be used to minimize } \\
\text { or eliminate job performance decrements resulting from drug or alcohol } \\
\text { abuse, are also discussed. }\end{array}$} \\
\hline \multicolumn{3}{|l|}{$\begin{array}{l}\text { 17. KEY WOADS AND DOCUMENT ANALYSIS } \\
\text { drug abuSe } \\
\text { alcohol abuse } \\
\text { nuclear utility industry } \\
\text { job performance } \\
\text { employee assistance programs }\end{array}$} \\
\hline \multicolumn{3}{|l|}{ T7b. IDENTIFIEAS: OPEN-EN DE D TERMS } \\
\hline \multirow{2}{*}{$\begin{array}{l}\text { 19. AVALLABILITY STATEMENT } \\
\text { Unlimited }\end{array}$} & $\begin{array}{l}\text { CLASS,iThy mont } \\
\text { SSified }\end{array}$ & 21 NO. OF PAGES 140 \\
\hline & $\begin{array}{l}\text { CLASS I They ow } \\
\text { SSified }\end{array}$ & $\begin{array}{c}22 \text { OAICE } \\
\text { S }\end{array}$ \\
\hline
\end{tabular}


